\title{
Endophytic fungi: a reservoir of antibacterials
}

\section{Sunil K. Deshmukh*, Shilpa A. Verekar and Sarita V. Bhave}

Department of Natural Products, Piramal Enterprises Limited, Mumbai, India

\section{Edited by:}

Luis Cláudio Nascimento Da Silva, University of Copenhagen, Denmark

\section{Reviewed by:}

Carsten Sanders, Kutztown

University, USA

Paras Jain, Albert Einstein College of

Medicine of Yeshiva University, USA

*Correspondence:

Sunil K. Deshmukh, Department of Natural Products, Piramal

Enterprises Limited, 1, Nirlon

Complex, Off Western Express

Highway, Near NSE Complex,

Goregaon (East), Mumbai 400 063,

India

e-mail: sunild2811@rediffmail.com
Multidrug drug resistant bacteria are becoming increasingly problematic particularly in the under developed countries of the world. The most important microorganisms that have seen a geometric rise in numbers are Methicillin resistant Staphylococcus aureus, Vancomycin resistant Enterococcus faecium, Penicillin resistant Streptococcus pneumonia and multiple drug resistant tubercule bacteria to name a just few. New drug scaffolds are essential to tackle this every increasing problem. These scaffolds can be sourced from nature itself. Endophytic fungi are an important reservoir of therapeutically active compounds. This review attempts to present some data relevant to the problem. New, very specific and effective antibiotics are needed but also at an affordable price! A Herculean task for researchers all over the world! In the Asian subcontinent indigenous therapeutics that has been practiced over the centuries such as Ayurveda have been effective as "handed down data" in family generations. May need a second, third and more "in-depth investigations?"

Keywords: endophytic fungi, antibacterial compounds, natural products, drug resistance, medicinal plants

\section{INTRODUCTION}

The last two decades have witnessed a rise in the numbers of Methicillin resistant Staphylococcus aureus (MRSA), Vancomycin resistant Enterococcus faecium (VRE) and Penicillin resistant Streptococcus pneumoniae (PRSP) and a variety of antibiotics (Menichetti, 2005). New drugs such as Linezolid and Daptomycin have already acquired resistance (Mutnick et al., 2003; Skiest, 2006). MDR- and XDR-TB (Gillespie, 2002; LoBue, 2009) are emerging global threats, being difficult to diagnose, expensive to treat and with variable results. Rice (2008) reported that the ESKAPE organism's E. faecium, S. aureus, Klebsiella pneumoniae, Acinetobacter baumanii, P. aeruginosa, and Enterobacter species are the main causative agents of infections in a majority of US hospitals. To combat all these continuing developments, a search for new and novel drugs scaffolds remains the high priority activity.

Eighty five years after the discovery of Penicillin in 1929, scientists all over the world continue to investigate natural products. The novelty of structures and scaffolds, their varied bioactivities plus their abilities to act as lead molecules is immense. According to Newman and Cragg (2012), in the years 1981-2010, 50\% of all small molecules originated from natural products. Mainly antibacterial, anticancer, antiviral and antifungals compounds from natural sources such as plant, fungi and bacteria themselves. The extraordinary advantages of natural products as sources of biotherapeutics is beyond question.

Though diverse chemical compounds with equally diverse scaffolds and bioactivities have been reported from fungi over the years, the vast group still remains to be fully exploited. Out of $\sim 1$ million different fungal species only $\sim 100,000$ have been described (Hawksworth and Rossman, 1997). Dreyfuss and Chapela (1994) estimated that endophytic fungi, alone could be $\sim 1$ million. The genetic diversity of fungal endophytes may be a major factor in the discovery of novel bioactive compounds (Gunatilaka, 2006). The true potential of these endophytes is yet to be trapped.

From the first reports of isolation from the Lolium temulentum typically known as Darnel (ryegrass) by Freeman (1904), to the latest one from Antarctic moss (Melo et al., 2014), endophytic fungi have attracted the attention of botanists, chemists, ecologists, mycologists, plant pathologists and pharmacologists. It is estimated that each and every of the almost 300,000 plants that exist, hosts one or more endophyte (Strobel and Daisy, 2003). They occur everywhere, from the Arctic to Antarctic and temperate to the tropical climates. Endophytes reside in internal tissues of living plants but this association does not cause any immediate, overt, negative effects on the host plant (Bacon and White, 2000). According to Aly et al. (2011), the endophyte-plant host relationship is a balanced symbiotic continuum, ranging from mutualism through commensalism to parasitism. Many endophytic fungi remain quiescent within their hosts until it stressed or begins to undergo senescence. At this juncture the fungi may turn pathogenic (Rodriguez and Redman, 2008).

The impact of endophytes on our lives is seen in several of ways; from an insecticidal bio fumigant from the Muscodor albus, against adults and larvae of potato tuber moths (Lacey and Neven, 2006) to synthesis of "myco-diesel" by Gliocladium roseum, in the hope of alternate fuel options (Strobel et al., 2008). Between these extremes, endophytes has been shown to produce several pharmacologically important compounds such as antimycotics Cryptocin (Li et al., 2000) and Ambuic acid (Li et al., 2001), anticancer Torreyanic acid (Lee et al., 1996), Taxol (Strobel et al., 1996), antiinflammatory Ergoflavin (Deshmukh et al., 2009), antidiabetic (nonpeptidal compound L-783,281) (Zhang et al., 1999), antioxidant Pestacin (Harper et al., 2003), Isopestacin (Strobel et al., 2002), antiviral Cytonic acids A and B (Guo et al., 2000), alkaloids 
and polyketides Sclerotinin A (Lai et al., 2013), Cryptosporioptide (Saleem et al., 2013), enzyme inhibitors- Fusaric acid derivatives (Chen et al., 2013), Anthraquinones (Hawas et al., 2012) and immunosuppressive agents Subglutinols A and B (Lee et al., 1995).

The need for novel antibacterials to combat this increasing variety of infections becomes a priority endeavor. Endophytic fungi may be an important source for such biotherapeutics like new antibacterials against Mycobacterium tuberculosis especially in poverty ridden tropical countries of Asia. Here the need could also involve a nutritional efforts to boost the immunity in the population. Many of the compounds with their host plants are shown in Table 1.

\section{ANTIBACTERIALS FROM ENDOPHYTIC FUNGI COMPOUNDS FROM ASCOMYCETES}

Ascomycetes are an important class of fungi where there is formation of ascospores. Some genera of this class are prolific producer of bioactive metabolites. The genus Pestalotiopsis exists as an endophyte in most of the world's rainforests and is extremely biochemically diverse. Some examples of products from this group are Ambuic acid (1) and its derivative (2) (Figure 1) isolated from a Pestalotiopsis sp. of the lichen Clavaroid sp. Compounds (1) and (2) are active against $S$. aureus (ATCC 6538) with $\mathrm{IC}_{50}$ values of 43.9 and $27.8 \mu \mathrm{M}$, respectively (the positive control Ampicillin showed an $\mathrm{IC}_{50}$ value of $1.40 \mu \mathrm{M}$ ) (Ding et al., 2009).

Pestalotiopen A (3) (Figure 1), from Pestalotiopsis sp. of the Chinese mangrove Rhizophora mucronata exhibited moderate antimicrobial activity against Enterococcus faecalis with an MIC value between 125 and $250 \mu \mathrm{g} / \mathrm{mL}$ (Hemberger et al., 2013).

A novel phenolic compound, 4-(2, 4, 7-trioxa-bicyclo [4.1.0] heptan-3-yl) phenol (4) (Figure 1) was isolated from Pestalotiopsis mangiferae associated with Mangifera indica. The compound exhibits activity against Bacillus subtilis and K. pneumoniae (MICs $0.039 \mu \mathrm{g} / \mathrm{ml}$ ), E. coli and Micrococcus luteus (MICs $1.25 \mu \mathrm{g} / \mathrm{ml}$ ) and P. aeruginosa (MIC $5.0 \mu \mathrm{g} / \mathrm{ml}$ ). The positive control (Gentamycin) is showed activity against $B$. subtilis, K. pneumoniae and M. luteus, E. coli, and P. aeruginosa (MICs range 5.0-10.0 $\mu \mathrm{g} / \mathrm{ml}$ ). Transmission electron microscopy (TEM) analysis for mode of action of compound (4) showed that against the three human pathogens (E. coli, P. aeruginosa, and $K$. pneumoniae), morphological alterations took place: such as destruction of bacterial cells by cytoplasmic agglutination and formation of pores in cell wall membranes (Subban et al., 2013).

Pestalone (5) (Figure 1) is a chlorinated benzophenone antibiotic produced by a co-cultured Pestalotia sp./Unicellular marine bacterium strain CNJ-328. Pestalotia sp. was isolated from the brown alga Rosenvingea sp. collected in the Bahamas Islands. Pestalone exhibits potent activity against MRSA (MIC $37 \mathrm{ng} / \mathrm{mL}$ ) and VRE (MIC $78 \mathrm{ng} / \mathrm{mL}$ ), indicating that Pestalone should be evaluated in advanced models of infectious disease (Cueto et al., 2001). It is active against $S$. aureus strain SG511, MRSA LT-1334 and Bacillus subtilis 168 with MICs of $3.1,6.25$, and $1.6 \mu \mathrm{g} / \mathrm{mL}$ respectively (Augner et al., 2013).

Phomopsis, another important genus exists as an endophyte in most plants and is also extremely biochemically diverse. Examples of bioactive metabolites from this endophyte are Dicerandrol A (6), B (7), and C (8) (Figure 1) from Phomopsis longicolla of the mint Dicerandra frutescens. They exhibit zones of inhibition of 11, 9.5, and $8.0 \mathrm{~mm}$ against B. subtilis respectively and 10.8, 9.5, and $7.0 \mathrm{~mm}$ respectively against $S$. aureus when tested at $300 \mu \mathrm{g} / \mathrm{disc}$ (Wagenaar and Clardy, 2001).

Dicerandrol C (8) (Figure 1) was isolated from Phomopsis longicolla strain C81, from the red seaweed Bostrychia radicans. Dicerandrol C (8) had significant antimicrobial activity against S. aureus (ATCC 6538) and Staphylococcus saprophyticus (ATCC 15305), with MICs of 1 and $2 \mu \mathrm{g} / \mathrm{mL}$ respectively (Erbert et al., 2012).

Dicerandrol A (6), Dicerandrol B (7), Dicerandrol C (8), Deacetylphomoxanthone B (9) and Fusaristatin A (10) (Figure 1) were isolated from Phomopsis longicolla S1B4 from a plant sample from Hadong-gun, Kyungnam Province, South Korea. All of the above compounds show moderate to low antibacterial activities against Xanthomonas oryzae KACC 10331 with MICs of 8, $16,>16,4$, and $128 \mu \mathrm{g} / \mathrm{mL}$ respectively. Dicerandrol A (6) is also active against $S$. aureus KCTC 1916, B. subtilis KCTC 1021, Clavibacter michiganesis KACC 20122, Erwinia amylovora KACC 10060 , with MICs value of $0.25,0.125,1.0$, and $32.0 \mu \mathrm{g} / \mathrm{mL}$ respectively (Lim et al., 2010). Monodeacetylphomoxanthone B (11) (Figure 1) was reported from the same culture along with compounds (6-9). It is active against X. oryzae with an MIC of $32 \mu \mathrm{g} / \mathrm{mL}$ (Choi et al., 2013).

Phomoxanthones A (12) and B (13) (Figure 1) were obtained from Phomopsis sp. BCC 1323, of the leaf of Tectona grandis L., from the Mee Rim district of Chaingmai Province, Northern Thailand. These compounds show significant "in vitro" antitubercular activities with MICs of 0.5 and $6.25 \mu \mathrm{g} / \mathrm{mL}$ respectively against Mycobacterium tuberculosis H37Ra strain, in comparison to isoniazide and kanamycin sulfate (MICs of 0.050 and $2.5 \mu \mathrm{g} / \mathrm{mL}$, respectively) that are used in clinics today (Isaka et al., 2001).

Phomoxanthone A (12) (Figure 1), was also isolated from a Phomopsis sp. of the stem of Costus sp. growing in the rain forest of Costa Rica. It has activity against Bacillus megaterium at a concentration of $10 \mathrm{mg} / \mathrm{mL}$ (radius of zone of inhibition of $3-4 \mathrm{~cm}$ ) (Elsaesser et al., 2005).

Cycloepoxylactone (14) (Figure 1) and cycloepoxytriol B (15) (Figure 2) were detected from Phomopsis sp. (internal strain no. 7233) of Laurus azorica. They are moderately active against B. megaterium (Hussain et al., 2009a).

Phomosines A-C (16-18) (Figure 2), three new biaryl ethers were obtained from Phomopsis sp. of the leaves of Teucrium scorodonia. All three compounds were moderately active against B. megaterium and E. coli in vitro, using $6 \mathrm{~mm}$ filter paper disc with $50 \mu \mathrm{l}$ each of a $15 \mathrm{mg} / \mathrm{mL}$ solution (Krohn et al., 1995). The same compounds were obtained from Phomosis sp. of Ligustrum vulgare and showed activity against $B$. megaterium in vitro with 10,10 , and $7 \mathrm{~mm}$ zone of inhibition using $6 \mathrm{~mm}$ filter paper disc and $50 \mu \mathrm{g}$ of compound $(50 \mu \mathrm{L}$ of $1 \mathrm{mg} / \mathrm{mL}$ ) respectively (Krohn et al., 2011).

Phomosine A (16) and Phomosine G (19) (Figure 2) were isolated from Phomopsis sp. of the halo tolerant plant Adenocarpus foliolosus from Gomera. Both the compound exhibited moderate 


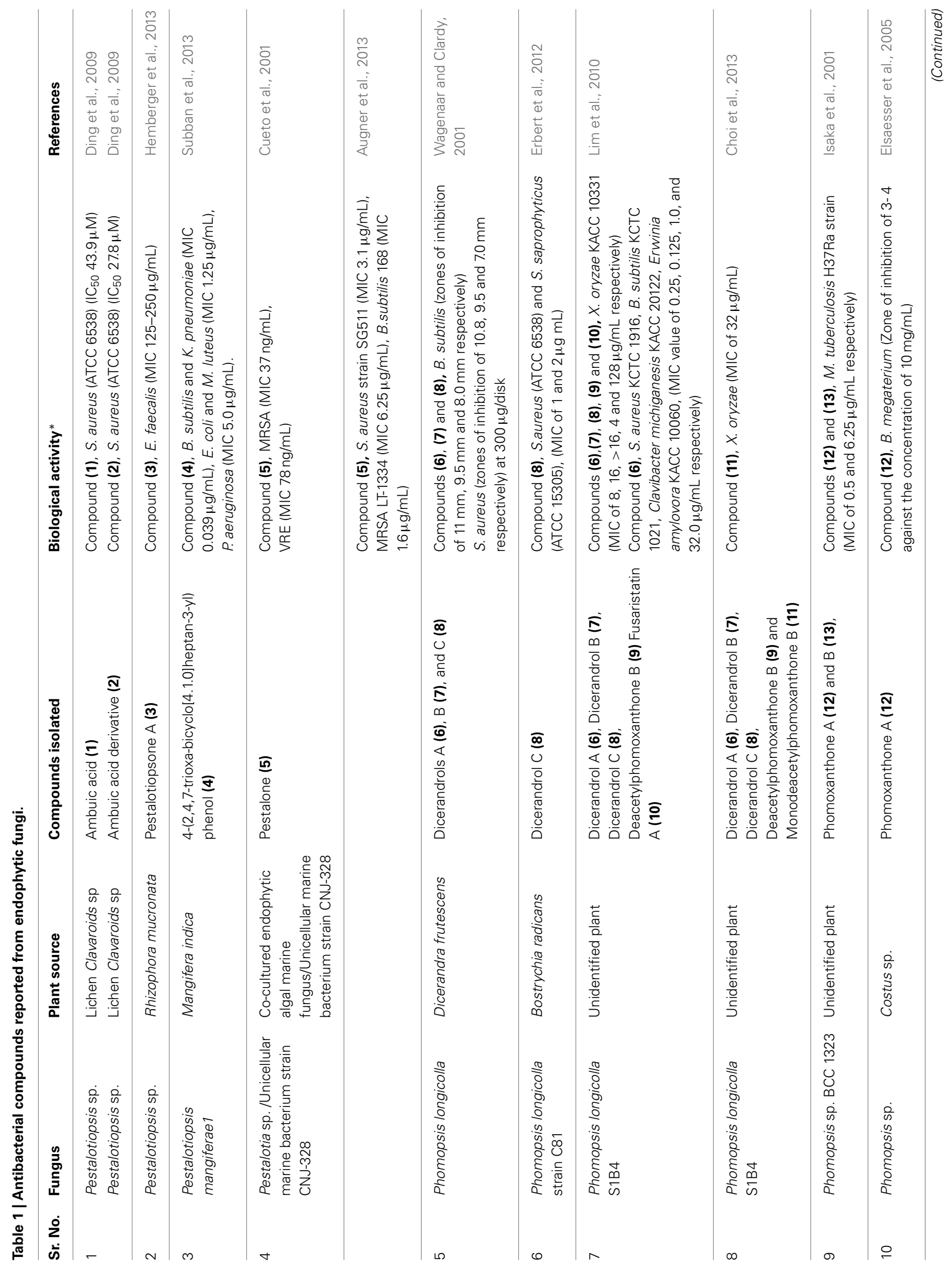




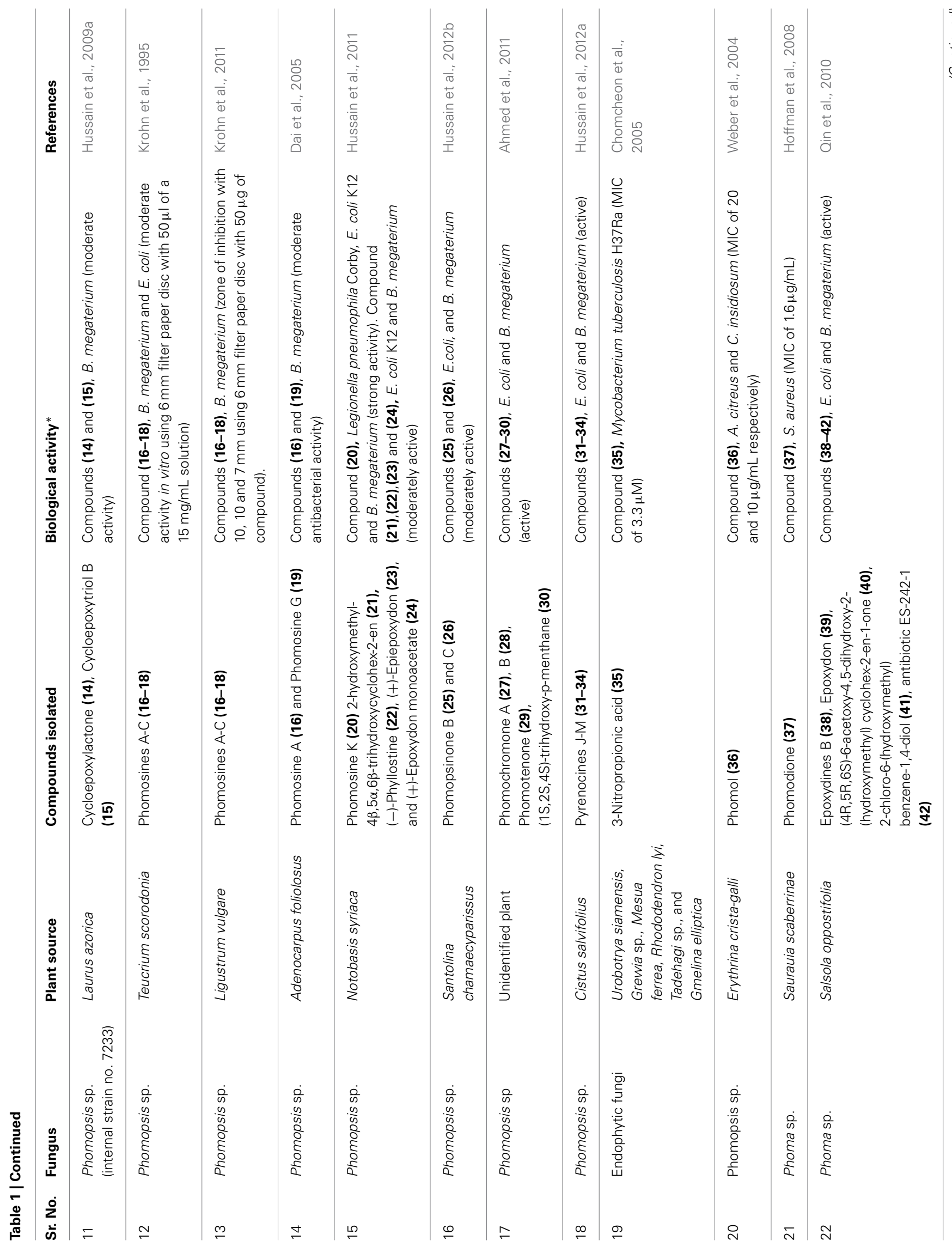




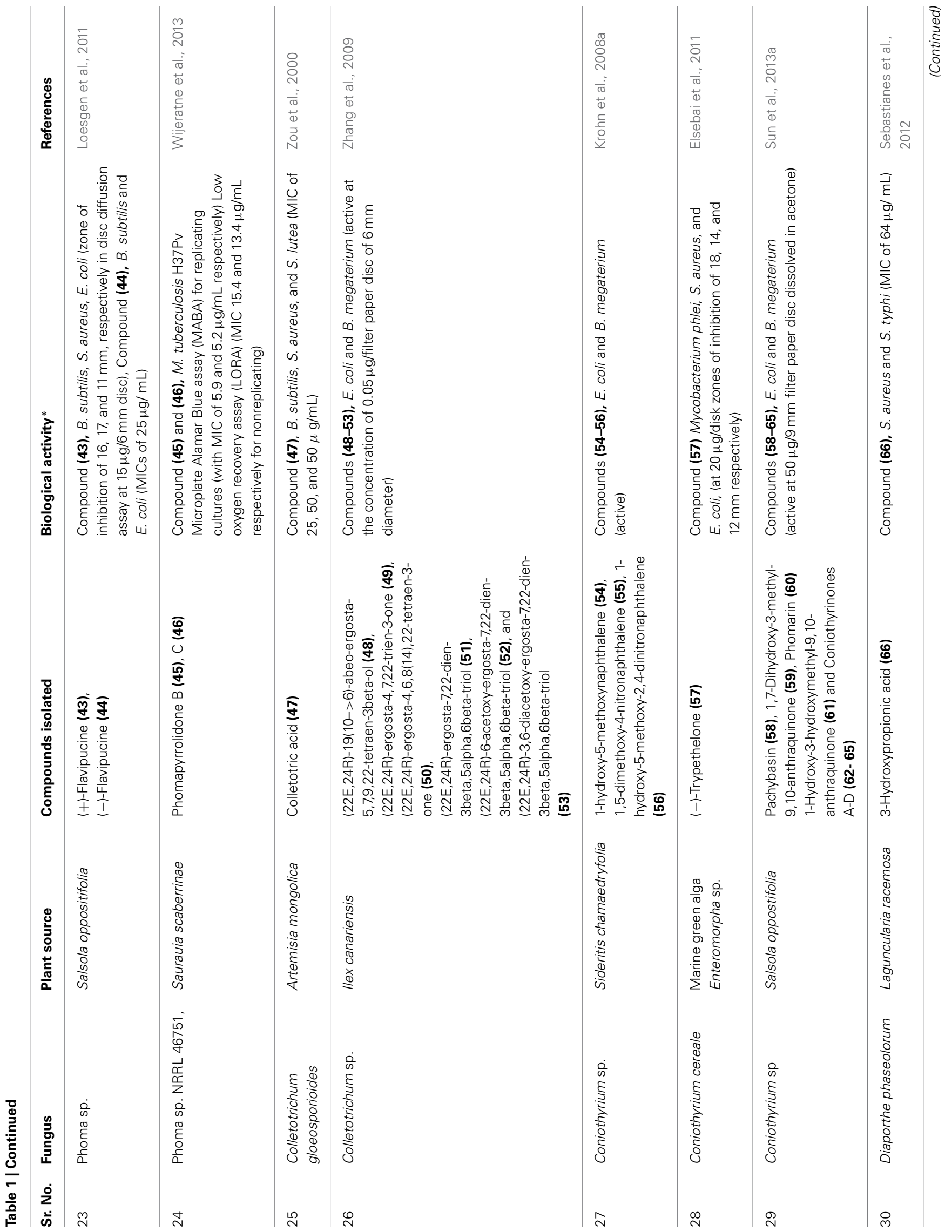




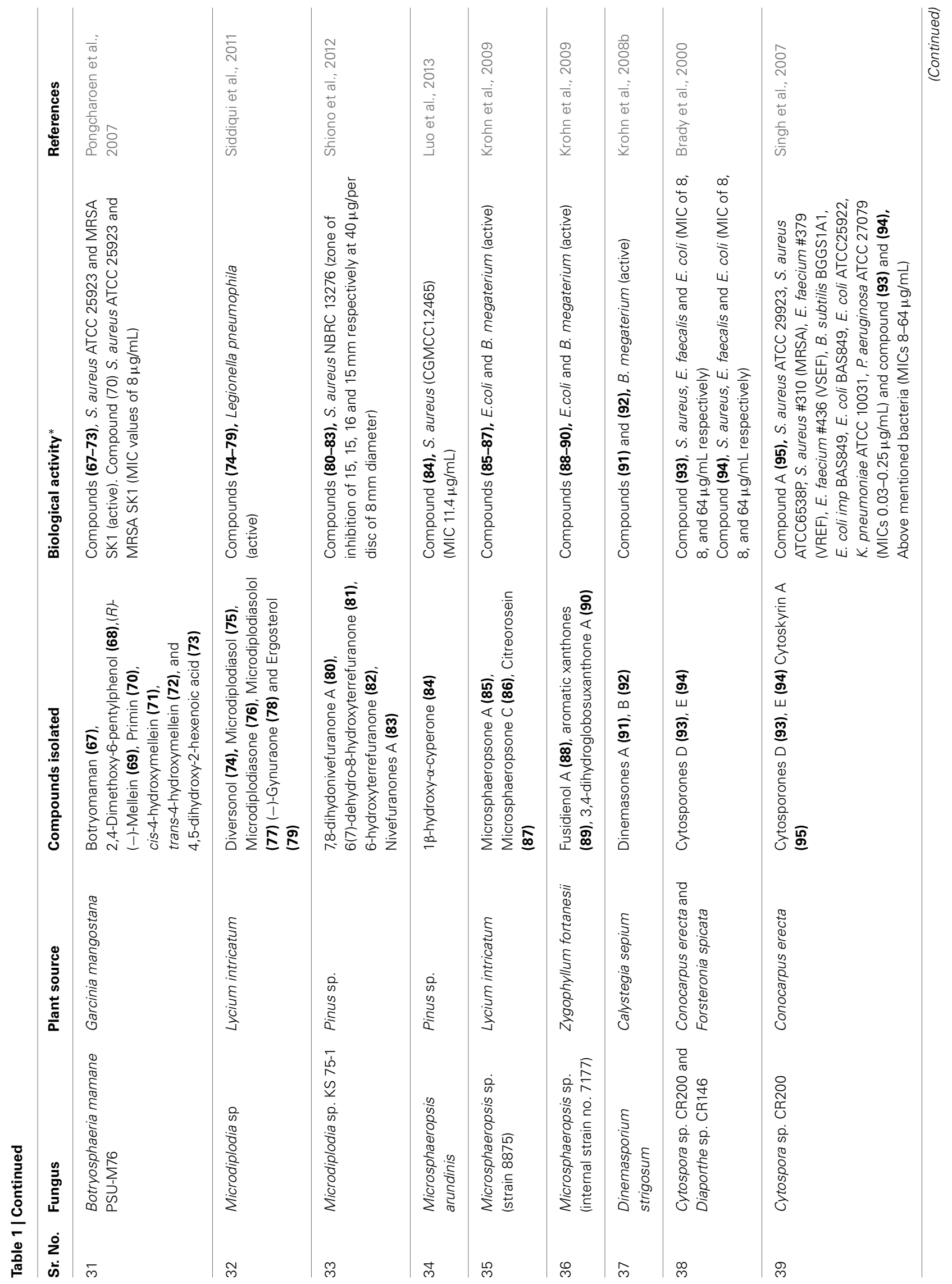




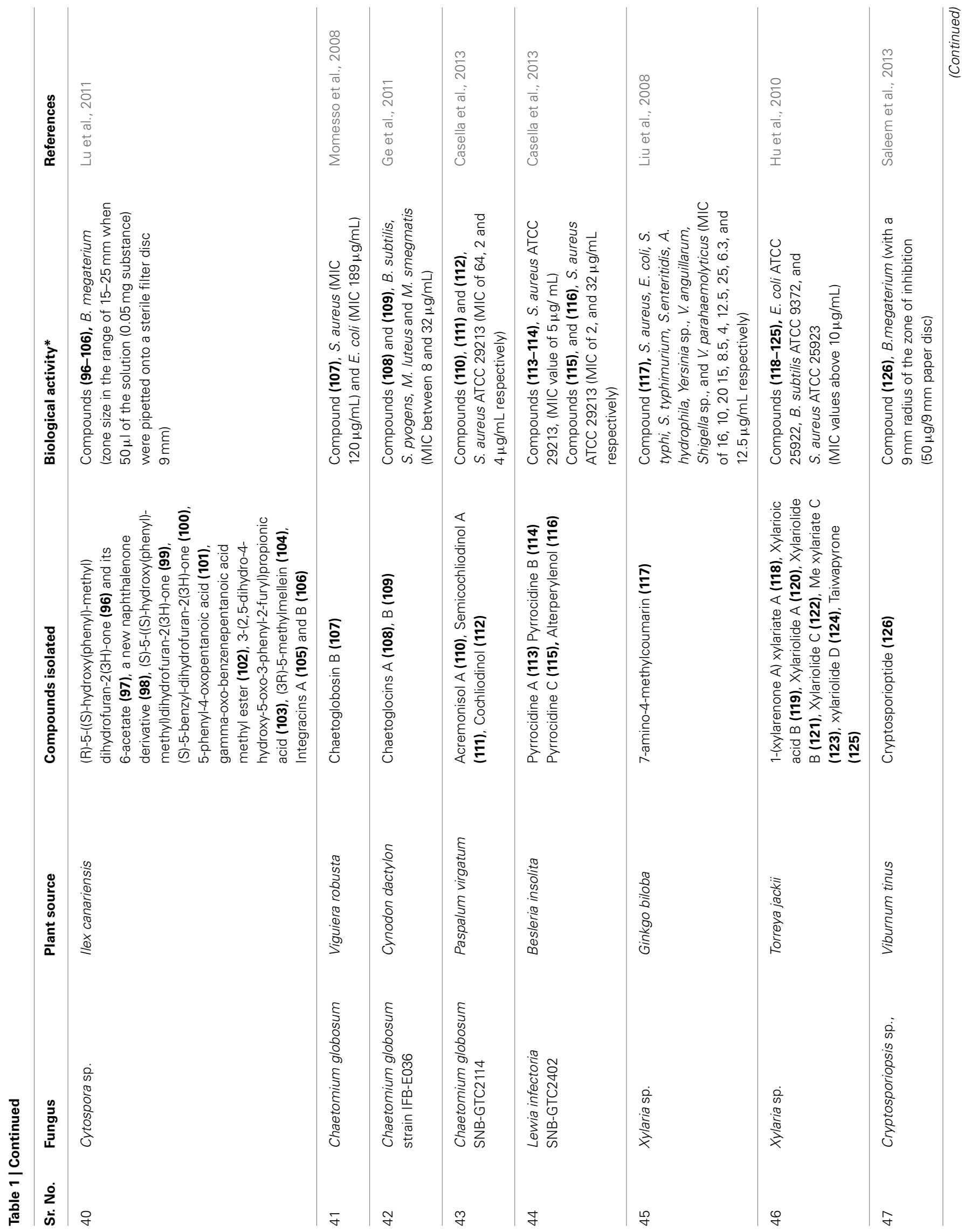




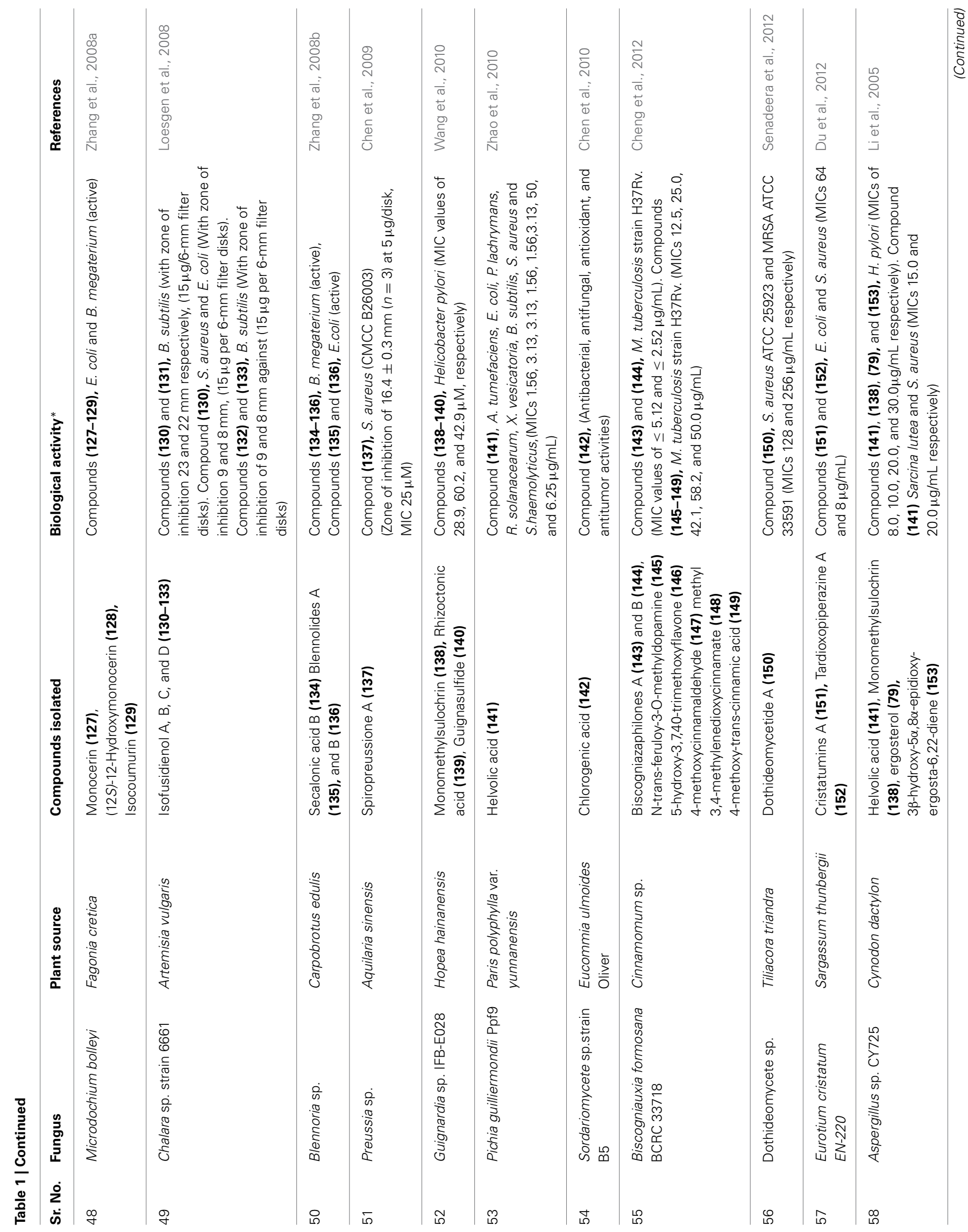




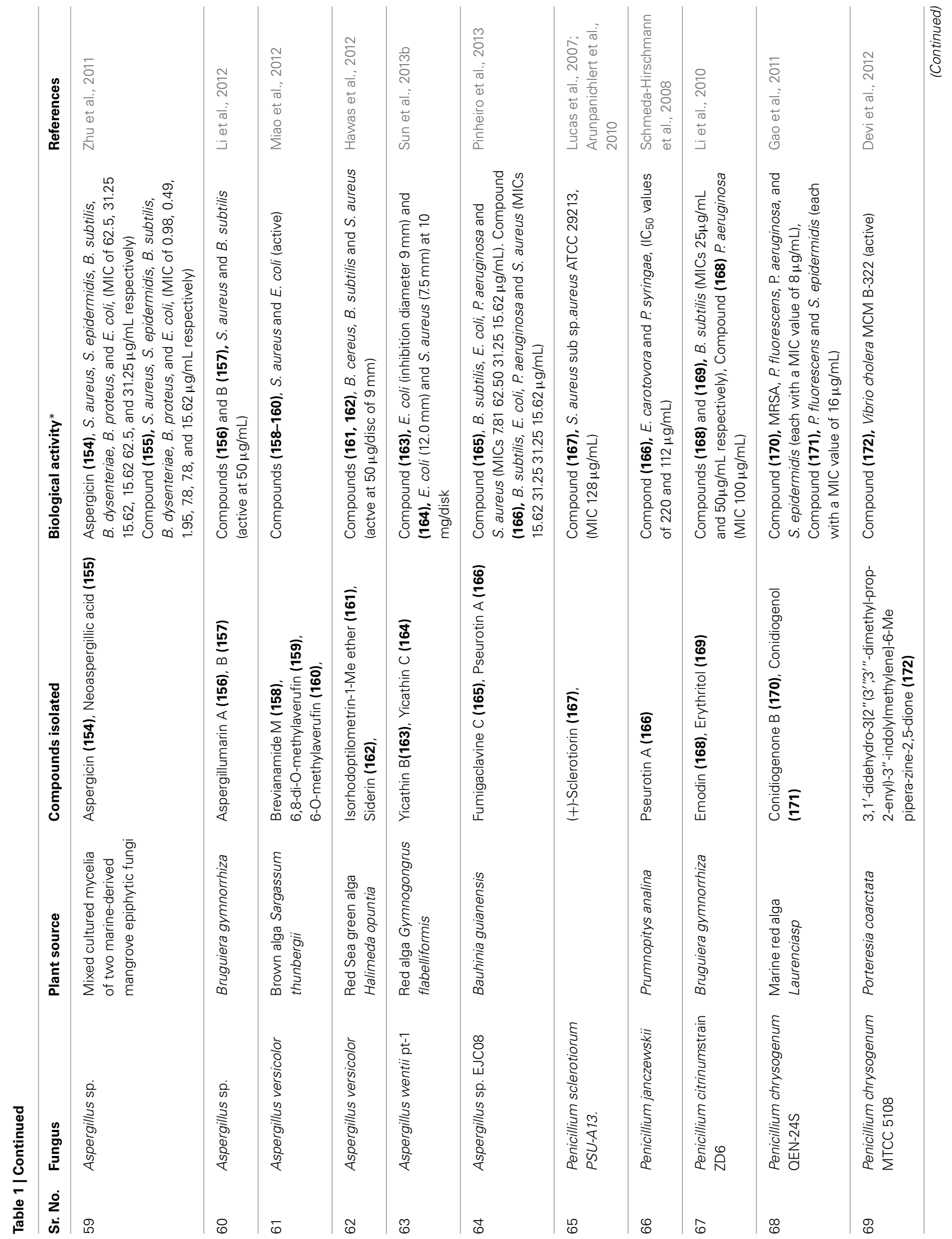




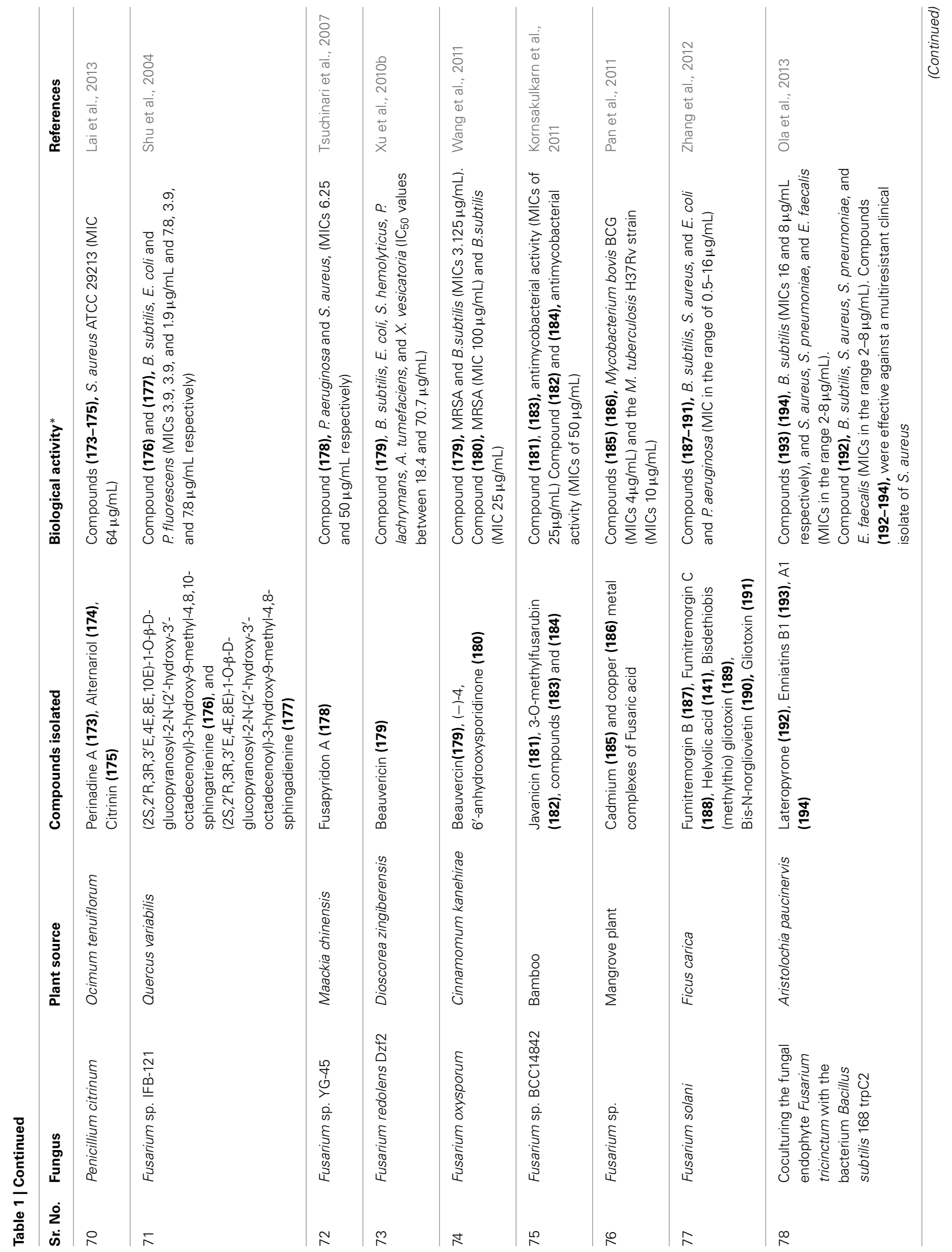




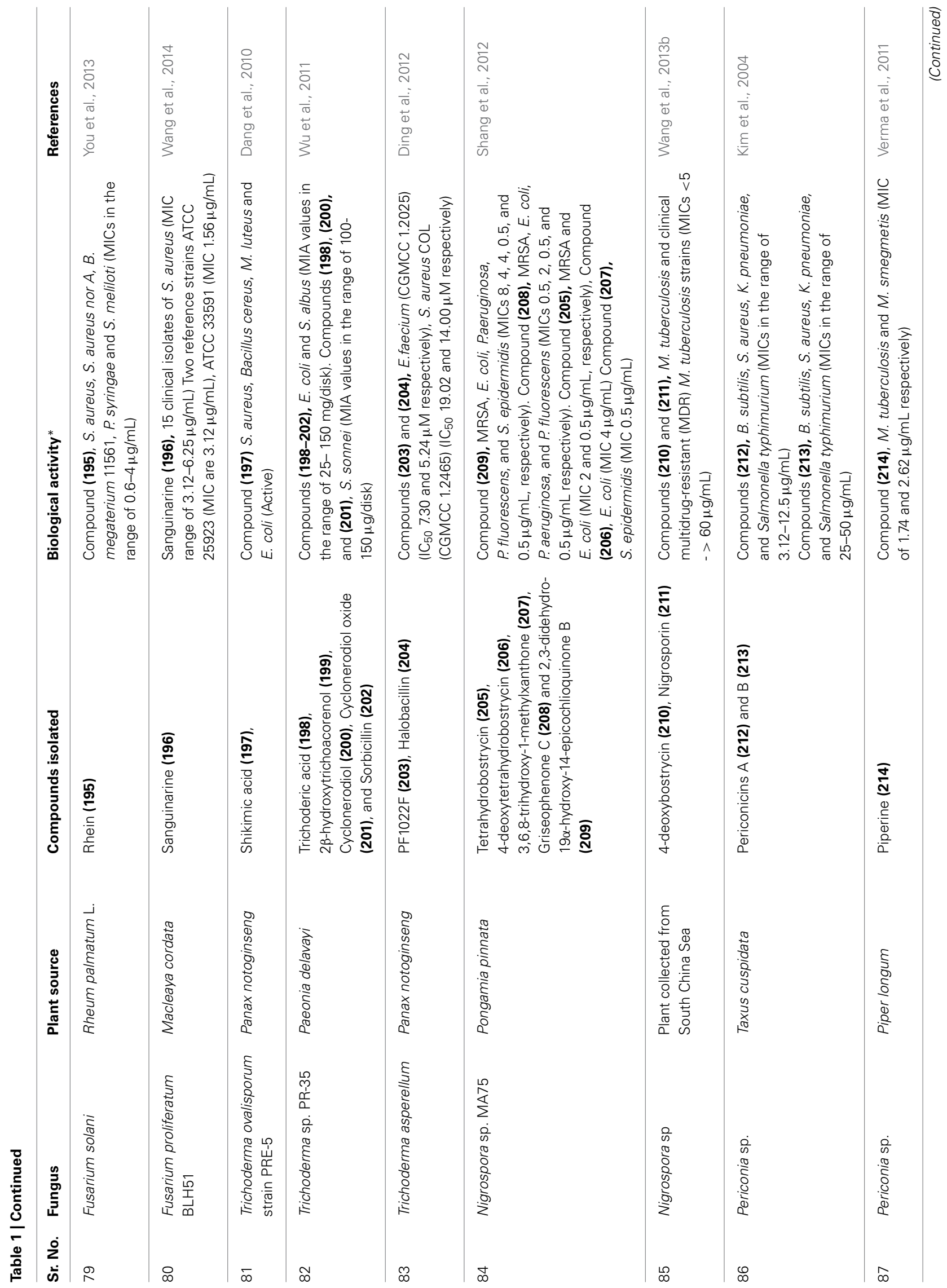




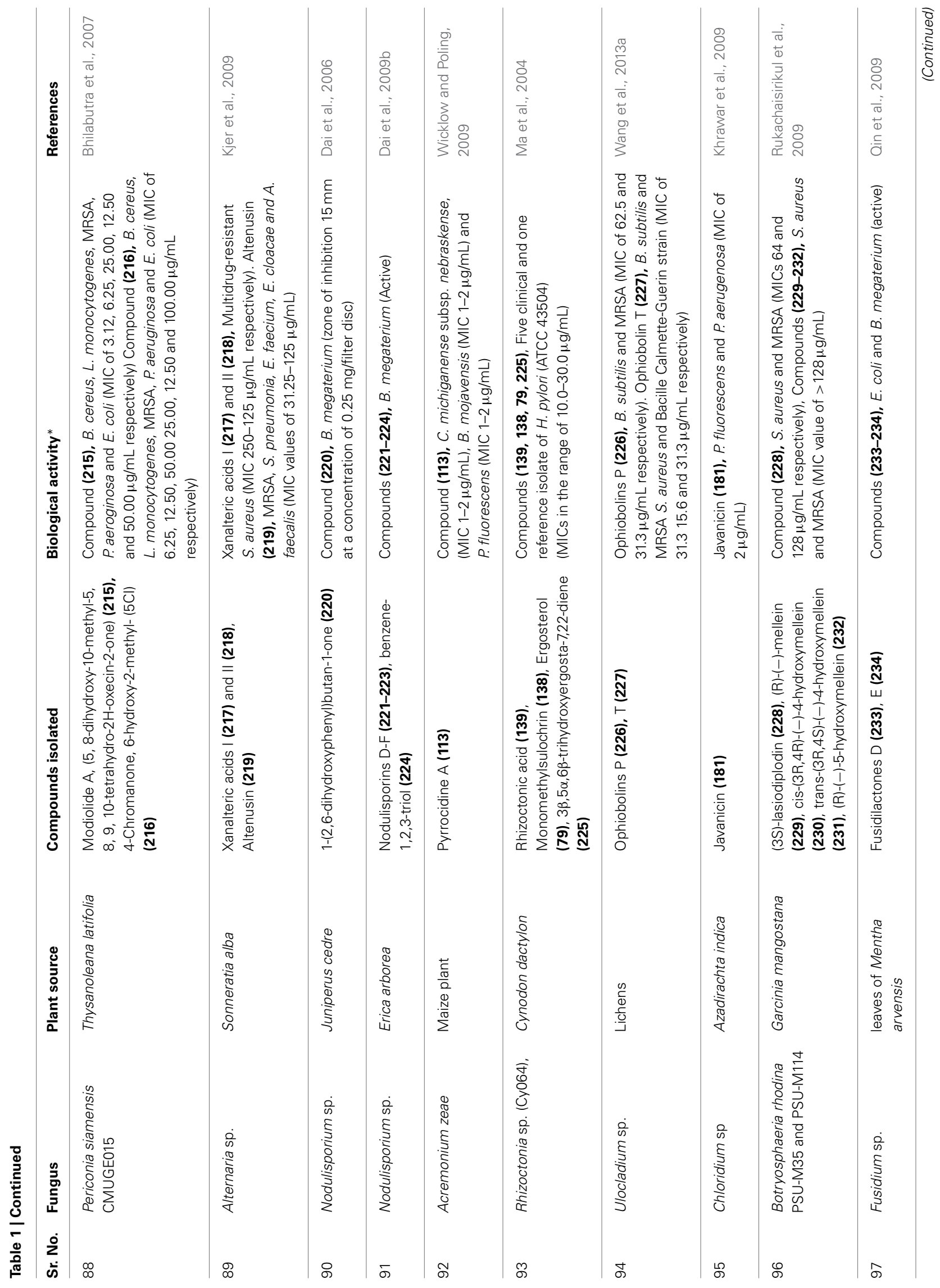




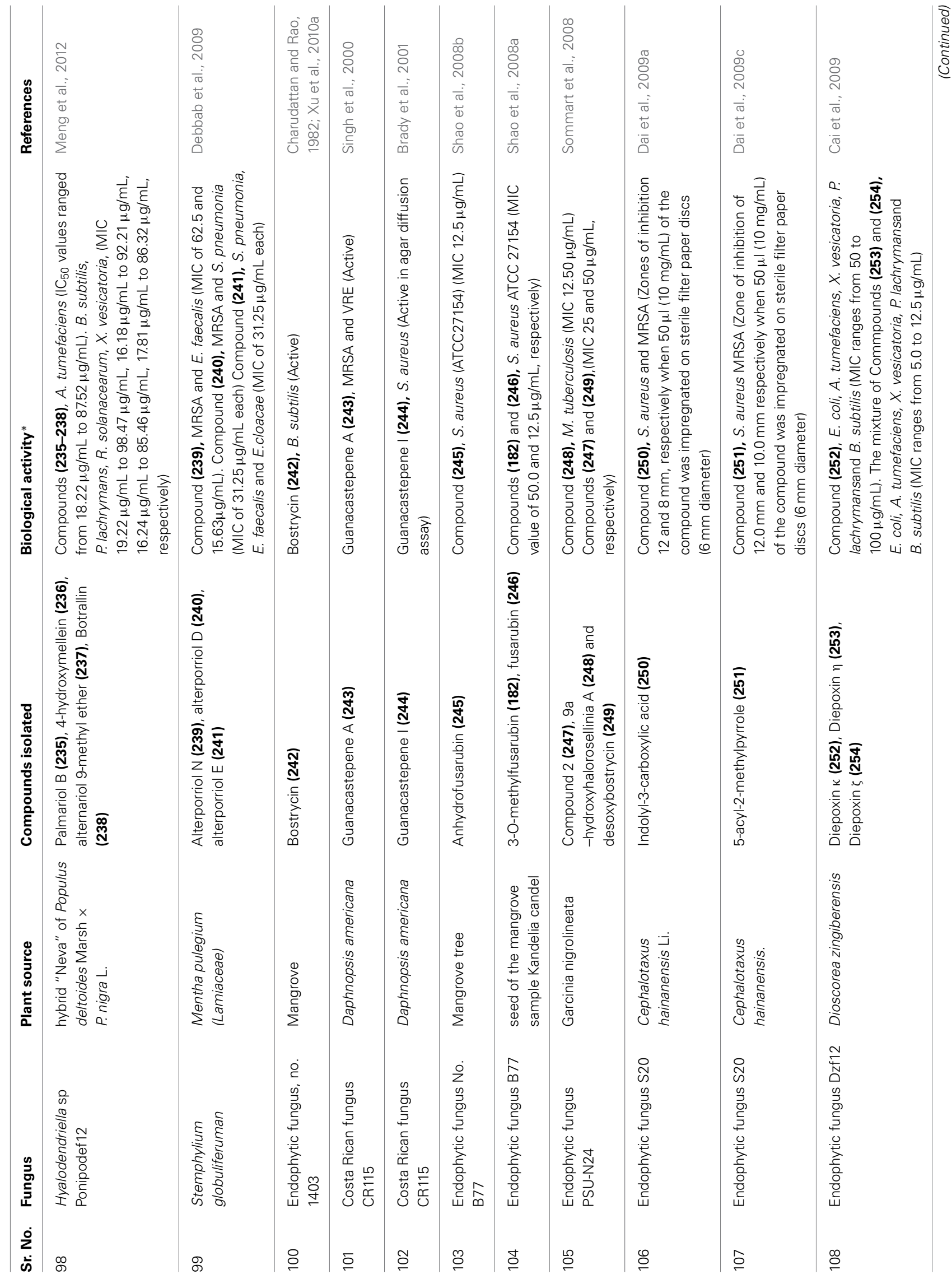


antibacterial activities against B. megaterium (Dai et al., 2005).

Phomosine K (20), 2-hydroxymethyl- $4 \beta, 5 \alpha, 6 \beta$-trihydroxycyclohex-2-en (21), (-)-Phyllostine (22), (+)-Epiepoxydon (23), and (+)-Epoxydon monoacetate (24) (Figure 2) were isolated from a Phomopsis sp. of Notobasis syriaca. Phomosine $\mathrm{K}$ (20) is active against Legionella pneumophila Corby, E. coli $\mathrm{K} 12$ and $B$. megaterium in vitro while 2-hydroxymethyl$4 \beta, 5 \alpha, 6 \beta$-trihydroxycyclohex-2-en (21), (-)-Phyllostine (22), (+)-Epiepoxydon (23), and (+)-Epoxydon monoacetate (24) showed moderate activities against E. coli $\mathrm{K} 12$ and B. megaterium (Hussain et al., 2011).

Phomopsinone B (25) and C (26) from a Phomopsis sp. present in stems of Santolina chamaecyparissus from Sardinia showed moderate activities against E.coli, and B. megaterium (Hussain et al., 2012b).

Phomochromone A (27), B (28), Phomotenone (29), and $(1 S, 2 S, 4 S)$-trihydroxy- $p$-menthane (30) (Figure 2) were isolated from a Phomopsis sp. of Cistus monspeliensis. All three compounds (27-30) show activity against E. coli and B. megaterium (Ahmed et al., 2011).

Pyrenocines J-M (31-34) (Figure 2) were isolated from a Phomopsis sp. of the plant Cistus salvifolius, internal strain 7852. All four compounds (31-34) are active against B. megaterium and E. coli (Hussain et al., 2012a).

3-Nitropropionic acid (35) (Figure 2) was isolated from several strains of endophytic fungus of the genus Phomopsis sp. obtained from six species of Thai medicinal plants (Table 1) from the forest areas of Chiangmai, Nakhonrachasima, and Pitsanulok Provinces of Thailand. 3-Nitropropionic acid exhibits potent activity against Mycobacterium tuberculosis H37Ra with the MIC of $3.3 \mu \mathrm{M}$, but no in vitro cytotoxicity was observed toward a number of cell lines (Chomcheon et al., 2005). 3-Nitropropionic acid is known to inhibit isocitrate lyase (ICL), an enzyme required for fatty acid catabolism and virulence in M. tuberculosis (MuñozElías and McKinney, 2005).

Phoma is another genus which produces diverse compounds. Here are some examples of bioactive compounds produced by this genus. Phomol (36) (Figure 3), a novel antibiotic, was isolated from a Phomopsis sp. of the medicinal plant Erythrina crista-galli. Phomol is active against Arthrobacter citreus and Corynebacterium insidiosum with MICs of 20 and $10 \mu \mathrm{g} / \mathrm{mL}$ respectively (Weber et al., 2004).

Phomodione (37), an usnic acid derivative was isolated from a Phoma sp. of Saurauia scaberrinae. Phomodione was found to be effective against $S$. aureus at a MIC of $1.6 \mu \mathrm{g} / \mathrm{mL}$ (Hoffman et al., 2008).

The antibacterials Epoxydine B (38), Epoxydon (39), (4R, 5R,6S)-6-acetoxy-4,5-dihydroxy-2-(hydroxymethyl)cyclohex-2-

en-1-one (40), 2-chloro-6-(hydroxymethyl)benzene-1,4-diol (41), and the antibiotic ES-242-1 (42) (Figure 3), were isolated from a Phoma sp. of Salsola oppostifolia. Compounds (38-42) show activity against E. coli and B. megaterium (Qin et al., 2010).

Antibacterials (+)-Flavipucine (43) and (-)-Flavipucine (44) (Figure 3), were isolated from a Phoma sp., of the plant Salsola oppositifolia. (+)-Flavipucine (43) is active against B. subtilis, 
<smiles>[R]CC1=C(/C=C/CCCCC)C(=O)[C@]2(C/C=C(\C)C(=O)O)O[C@@H]2[C@@H](O)[C@@H]1O</smiles>

R= H Ambuic Acid (1)

$\mathbf{R}=$ Ac Ambuic acid derivative (2)

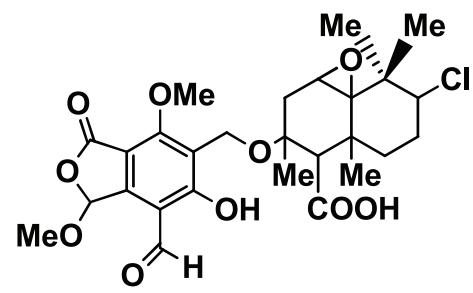

Pestalotiopen A (3)<smiles>Oc1ccc(C2OC[C@H]3O[C@H]3O2)cc1</smiles>

4-(2,4,7-trioxa-bicyclo[4.1.0] heptan-3-yl) phenol (4)<smiles>COc1c(Cl)c(C)c(Cl)c(O)c1C(=O)c1c(O)c(O)cc(O)c1CC=C(C)C</smiles>

Pestalone (5)<smiles>[R]OC[C@]12Oc3ccc(-c4ccc5c(c4O)C(=O)C4=C(O)C[C@H](C)[C@H](OC(C)=O)[C@@]4(O[R])O5)c(O)c3C(=O)C1=C(O)C[C@H](C)[C@H]2OC(C)=O</smiles>

$\mathbf{R}_{\mathbf{1}}=\mathbf{R}_{\mathbf{2}}=\mathrm{H}$ Dicerandrol A (6) $\mathbf{R}_{1}=$ Ac, $\mathbf{R}_{\mathbf{2}}=$ H Dicerandrol B (7) $\mathbf{R}_{\mathbf{1}}=\mathbf{R}_{\mathbf{2}}=$ Ac Dicerandrol C (8)<smiles></smiles>

Deacetyl phomoxanthone B (9)

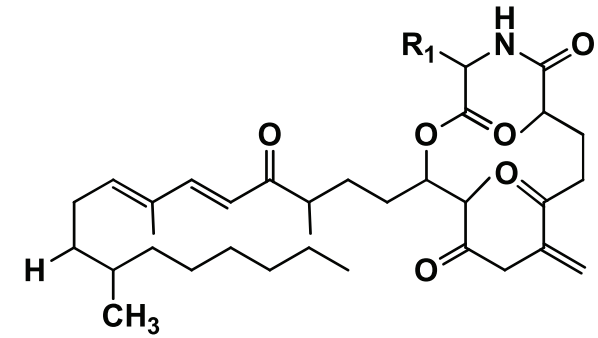<smiles>[R1][Z20]CCC(N)=O</smiles>

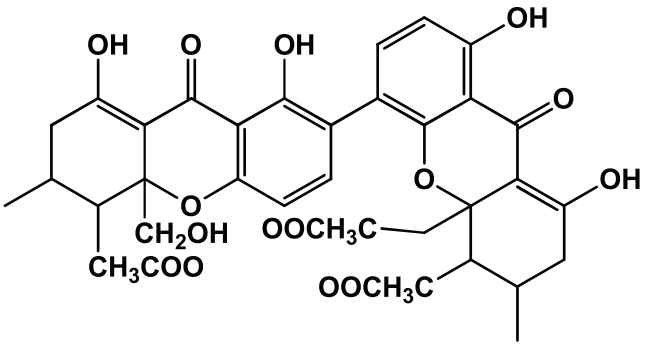

Monodeacetylphomoxanthone B (11)

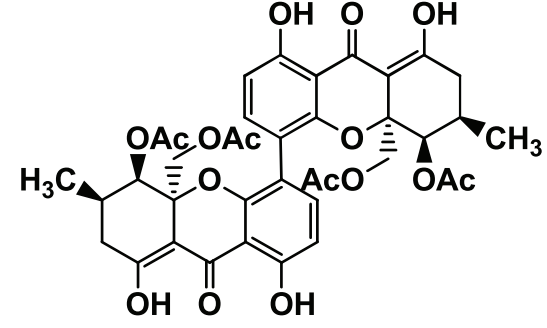

Phomoxanthone A (12)<smiles></smiles>

Phomoxanthone B (13)<smiles>CCC[C@H]1CC2=C(C(=O)O1)[C@@H](O)[C@H]1O[C@H]1[C@H]2O</smiles>

Cycloepoxylactone (14)

FIGURE 1 | Structures of antibacterial metabolites isolated from Ascomycetes (1-14).

S. aureus, E. coli with inhibition zones of 16,17 , and $11 \mathrm{~mm}$, respectively in disc diffusion assay at $15 \mu \mathrm{g} / 6 \mathrm{~mm}$. (-)Flavipucine (44) was active against B. subtilis and E. coli at MIC of $25 \mu \mathrm{g} / \mathrm{mL}$ (Loesgen et al., 2011).
Three new alkaloids, Phomapyrrolidones B-C (45-46) (Figure 3), were isolated from a Phoma sp. NRRL 46751, of the plant Saurauia scaberrinae. Phomapyrrolidones B (45) and C (46) show weak in vitro activities when tested in microplate Alamar 
<smiles>CCC[C@H]1OCC2=C([C@H](O)[C@@H]3O[C@H]3[C@@H]2O)[C@H]1O</smiles>

Cycloepoxytriol B (15)<smiles>[R]c1c(O)cc(C)cc1Oc1c(C)c(C(=O)OC)c(O)c(C)c1O</smiles>

R= CHO Phomosine A (16) $\mathrm{R}=$ MePhomosine K (20)<smiles>COC(=O)c1c(O)cc(O)c(Oc2cc(C)cc(O)c2C=O)c1C</smiles>

Phomosine C (18)<smiles>COC(=O)c1c(C)c2c(c(C)c1O)OCc1c(O)cc(C)cc1O2</smiles>

Phomosine G (19)<smiles>O=C1C=C(CO)C(=O)[C@H]2O[C@H]12</smiles>

(-)- Phyllostine (22)<smiles>[R20]CC1=C[C@@H](O)[C@H]2O[C@H]2C1=O</smiles>

$\mathbf{R}=\mathbf{H}(+)$ - Epiepoxydon (23) $\mathbf{R}=\mathrm{Ac}(+)-$ Epoxydon monoacetate (24)<smiles>COCc1c(O)cc(C)cc1Oc1c(C)c(C(=O)OC)c(O)c(C)c1O</smiles>

Phomosine B (17)<smiles>O=C1C(CO)=C[C@@H](O)[C@H](O)[C@H]1O</smiles>

2-hydroxymethyl-4 $\beta, 5 \alpha$, 6 $\beta$-trihydroxy-cyclohex-2-en (21)<smiles>[R]C(C)C[C@H]1OCc2c(OC)cc(=O)oc2[C@H]1[R]</smiles>

$\mathrm{R}=\mathrm{H}, \mathrm{R} 1=\beta-\mathrm{OH}$ Phomopsinone B (25 ) $\mathrm{R}=\mathrm{H}, \mathbf{R} 1=\alpha-\mathrm{OH}$ Phomopsinone $\mathrm{C}$ (26)<smiles>Cc1cc2c(c(C)c1O)O[C@H](C)CC2=O</smiles>

Phomochromone A (27)<smiles>Cc1c(C)c2c(c(C)c1O)O[C@H](C)[C@@H](O)C2=O</smiles>

Phomochromone B (28)<smiles>CCC[C@H](O)C1=C(C)C(=O)C[C@H]1C</smiles>

Phomotenone (29)<smiles>CC(C)[C@]1(O)CC[C@@](C)(O)[C@H](O)C1</smiles>

(1S,2S,4S)-trihydroxy-pmenthane (30)<smiles>[R]C([R2])C[C@H]1Cc2oc(=O)cc(OC)c2CO1</smiles><smiles>[R]C(CCC)Cc1oc(=O)cc(OC)c1CO</smiles>

R=OAc Pyrenocine L (33) $\mathbf{R}=$ H Pyrenocine $M$ (34)<smiles>O=C(O)CC[N+](=O)[O-]</smiles>

3-Nitropropionic acid (35)

$\mathbf{R}_{1}=*=\mathrm{O}^{*}$ (keto), $\mathbf{R}_{2}=\mathrm{CH} 3$ Pyrenocine $\mathrm{K}$ (32)

FIGURE 2 | Structures of antibacterial metabolites isolated from Ascomycetes (15-35). 
<smiles>CCCCCC1OC(=O)CC(O)C(O)C=CC(O)C1OC(=O)C(C)=CC(C)CC</smiles>

Phomol (36)<smiles>O=C1C(CO)=C[C@@H](O)[C@H]2O[C@H]12</smiles>

Epoxydon (39)<smiles>COc1c(C)c(O)c(C(C)=O)c2c1[C@@]1(C)C(=O)C(C(C)=O)C(=O)C[C@]1(OC)O2</smiles>

Phomodione (37)<smiles>CC(=O)O[C@H]1C(=O)C(CO)=C[C@H](O)[C@@H]1O</smiles>

(4R,5R,6S)-6-acetoxy-4,5dihydroxy-

2-(hydroxymethyl)cyclohex-2-en-1-one (40)<smiles>O=C(O[C@H]1C(=O)C(CO)=C[C@H](O)[C@@H]1O)c1ccco1</smiles>

Epoxydine B (38)<smiles>OCc1cc(O)cc(Cl)c1O</smiles>

2-chloro-6-(hydroxymethyl) benzene-1,4-diol (41)<smiles>COc1cc(OC)c2c(O)c3c(c(-c4c5c(c(O)c6c(OC)cc(OC)cc46)CO[C@@H](C)[C@@H]5OC(C)C)c2c1)C[C@@H](C)OC3</smiles><smiles>CC1=CC(=O)[C@@]2(O[C@@H]2C(=O)CC(C)C)C(=O)N1</smiles>

(+)-Flavipucine (43)<smiles>CC1=CC(=O)[C@@]2(O[C@@H]2C(=O)CC(C)C)C(=O)N1</smiles>

(-)-Flavipucine (44)

ES-242-1 (42)

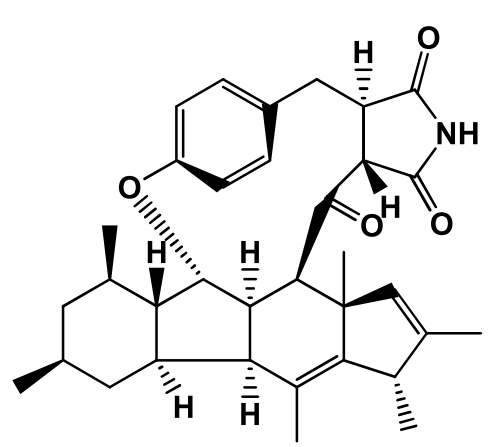

Phomapyrrolidone B (45)

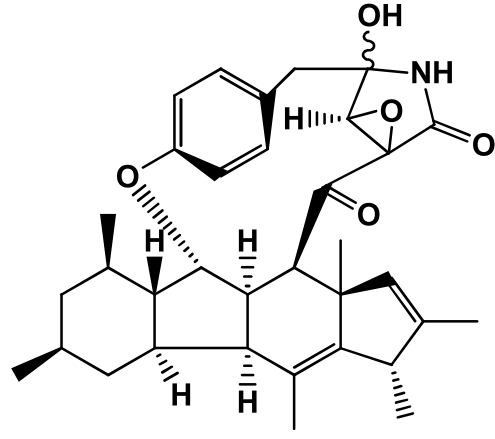<smiles>COc1c(C)c(OC(=O)c2c(C)cc(O)cc2O)c(C)c(C(=O)Oc2cc(O)c(C(=O)O)c(C)c2C)c1C</smiles>

Colletotric Acid (47)

FIGURE 3 | Structures of antibacterial metabolites isolated from Ascomycetes (36-47). 
Blue assays (MABA) with MICs of 5.9 and $5.2 \mu \mathrm{g} / \mathrm{mL}$ respectively and in the low oxygen recovery assay (LORA) with MICs of 15.4 and $13.4 \mu \mathrm{g} / \mathrm{mL}$ respectively, for nonreplicating $M$. tuberculosis H37Pv (Wijeratne et al., 2013).

Other endophytes of Ascomycetes are also known to produce antibacterials. For example Colletotric acid (47) (Figure 4) from Colletotrichum gloeosporioides of Artemisia mongolica or Nanjing, China inhibits B. subtilis, S. aureus, and Sarcina lutea with MICs of 25,50 , and $50 \mu \mathrm{g} / \mathrm{mL}$, respectively (Zou et al., 2000).
Antibacterials (22E,24R)-19(10->6)-abeo-ergosta-5,7,9,22tetraen-3 $\beta$-ol(48), (22E,24R)-ergosta-4,7,22-trien-3-one(49), (22E,24R)-ergosta-4,6,8(14),22-tetraen-3-one (50), (22E,24R)ergosta-7,22-dien-3 $\beta, 5 \alpha, 6 \beta$-triol (51),(22E,24R)-6-acetoxyergosta-7,22-dien-3 $\beta, 5 \alpha, 6 \beta$-triol (52), and (22E,24R)-3,6diacetoxy-ergosta-7,22-dien-3 $\beta, 5 \alpha, 6 \beta$-triol (53) (Figure 4), were isolated from a Colletotrichum sp. of Ilex canariensis from Gomera. Compounds (48-53) are active against E. coli and B. megaterium of $0.05 \mu \mathrm{g} /$ filter paper disc of $6 \mathrm{~mm}$<smiles>Cc1cc2c(c3c1C[C@H](O)CC3)CC[C@@]1(C)[C@@H](C)CC[C@H]1C2</smiles>

(22E,24R)-19(10 $\rightarrow 6)$-abeo-ergosta$5,7,9,22$-tetraen-3 $\beta$-ol (48)<smiles>CC(C)[C@H](C)/C=C/[C@@H](C)[C@H]1CCC2C3=CCC4=CC(=O)CC[C@]4(C)C3CC[C@]21C</smiles>

(22E,24R)-ergosta-4,7, 22-trien-3-one (49)<smiles>CC(C)C/C=C/[C@H](C)[C@H]1CCC2=C3C=CC4=CC(=O)CC[C@]4(C)C3CC[C@]21C</smiles>

(22E,24R)-ergosta-4,6,8 (14), 22-tetraen-3-one (50)

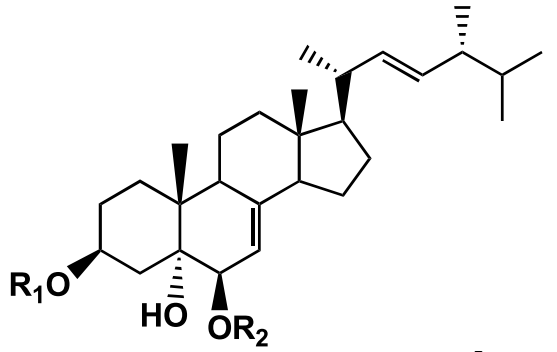<smiles>COc1cccc2c(O)cccc12</smiles><smiles>COc1ccc([N+](=O)[O-])c2c(OC)cccc12</smiles>

$\mathbf{R}_{1}=\mathbf{R}_{2}=\mathrm{H}$ (22E,24R)-ergosta-7,22-dien-3, 1-hydroxy-5-methoxynaphthalene (54) 1,5-dimethoxy-4-nitronaphthalene (55) $5 \alpha, 6 \beta$-triol (51)

$\mathbf{R}_{1}=\mathbf{H}, \mathbf{R}_{2}=$ Ac (22E,24R)-6-acetoxy-ergosta-7, 22-dien-3 $\beta, 5 \alpha, 6 \beta$-triol (52) $\mathbf{R}_{\mathbf{1}}=\mathbf{R}_{\mathbf{2}}=$ Ac (22E,24R)-3,6-diacetoxy-ergosta-7, 22-dien-3 $\beta, 5 \alpha, 6 \beta$-triol (53)<smiles>[R]c1cc(O)c2c(c1)C(=O)c1cc([R2])c([R])c([R4])c1C2=O</smiles>

$\mathbf{R}_{1}=\mathrm{CH}_{3}, \mathbf{R}_{\mathbf{2}}=\mathbf{R}_{\mathbf{3}}=\mathbf{R}_{\mathbf{4}}=\mathrm{H}$ Pachybasin (58) $\mathbf{R}_{1}=\mathrm{CH}_{3}, \mathbf{R}_{\mathbf{2}}=\mathbf{R}_{\mathbf{4}}=\mathrm{H}, \mathrm{R}_{\mathbf{3}}=\mathrm{OH}$ 1,7-dihydroxy -3-methyl-9,10-anthroquinone (59) $\mathbf{R}_{1}=\mathrm{CH}_{3}, \mathbf{R}_{2}=\mathrm{OH}, \mathbf{R}_{4}=\mathbf{R}_{3}=\mathrm{H}$ Phomarin (60) $\mathrm{R}_{\mathbf{1}}=\mathrm{CH}_{\mathbf{2}} \mathrm{OH}, \mathrm{R}_{\mathbf{2}}=\mathrm{R}_{\mathbf{3}}=\mathrm{R}_{\mathbf{4}}=\mathrm{H}$ 1-hydroxy -3-hydroxymethyl-9,10-anthroquinone (61)<smiles>COc1cccc2c(O)c([N+](=O)[O-])cc([N+](=O)[O-])c12</smiles>

1-hydroxy-5-methoxy2,4-dinitronaphthalene (56)<smiles>Cc1cc(O)cc2c1C1=C(C(=O)C2=O)C(C)(C)[C@@H]1C</smiles>

(-)-Trypethelone (57)<smiles>Cc1cc(O)c2c(c1)[C@H](O)[C@H]1C=C[C@@H](O)[C@H](O)[C@H]1C2=O</smiles>

Coniothyrinone A (62)<smiles>[R]C1C[C@H]2C(=O)c3c(O)cc(C)cc3C([R3])([R2])[C@@]2([R3])C[C@@H]1O</smiles>

$\mathbf{R}_{1}=\mathbf{R}_{3}=H, R_{2}=O H$ Coniothyrinone B (63)

$R_{1}=R_{3}=H, R_{2}=H$ Coniothyrinone $C(64)$

FIGURE 4 | Structures of antibacterial metabolites isolated from Ascomycetes (48-64). 
diameter (Zhang et al., 2009). Antibacterial 1-hydroxy-5-methoxynaphthalene (54), 1,5-dimethoxy-4-nitronaphthalene (55), 1-hydroxy-5-methoxy-2,4-dinitronaphthalene (56) (Figure 4), were isolated from Coniothyrium sp. internal strain number 7721 of the shrub Sideritis chamaedryfolia, from an arid zone near Alicante, Spain. These compounds were active against B. megaterium and E. coli (Krohn et al., 2008a).

(-)-Trypethelone (57), isolated from endophyte Coniothyrium cereale of the marine green alga Enteromorpha sp. showed activity against Mycobacterium phlei, S. aureus, and E. coli, at $20 \mu \mathrm{g} /$ disk with inhibition zones of 18,14 , and $12 \mathrm{~mm}$, respectively (Elsebai et al., 2011).

Antibacterials Pachybasin (58), 1, 7-Dihydroxy-3-methyl-9, 10-anthraquinone (59), Phomarin (60), 1-Hydroxy-3hydroxymethyl-9, 10-Anthraquinone (61), and Coniothyrinones A-D (62-65) (Figures 4, 5), were isolated from Coniothyrium sp., an endophyte of Salsola oppostifolia from Gomera in the Canary Islands. Compounds (58-65) were active against E. coli and $B$. megaterium in vitro in disc diffusion assay at $50 \mu \mathrm{g} / 9 \mathrm{~mm}$ disc dissolved in acetone (Sun et al., 2013a).

3-Hydroxypropionic acid (3-HPA) (66) (Figure 5) was isolated from the mangrove endophyte Diaporthe phaseolorum, from branches of Laguncularia racemosa, growing in Bertioga, located in south eastern Brazil. 3-HPA was active against both S. aureus and Salmonella typhi at an MIC of $64 \mu \mathrm{g} / \mathrm{mL}$ (Sebastianes et al., 2012).

Botryomaman (67), 2, 4-Dimethoxy-6-pentylphenol (68), (R)-(-)-Mellein (69), Primin (70), cis-4-hydroxymellein (71), trans-4-hydroxymellein (72) and 4, 5-dihydroxy-2-hexenoic acid (73) (Figure 5) were isolated from the endophyte Botryosphaeria mamane PSU-M76 from the leaves of Garcinia mangostana, collected in Suratthani Province, Thailand. The compounds were active against $S$. aureus ATCC 25923 and MRSA SK1. Primin was the most active with MIC values of $8 \mu \mathrm{g} / \mathrm{mL}$ against both the strains (Pongcharoen et al., 2007).

Microdiplodia sp. isolated from the shrub Lycium intricatum gave Diversonol (74), Microdiplodiasol (75), Microdiplodiasone (76), Microdiplodiasolol (77), (-)-Gynuraone (78), and Ergosterol (79) (Figure 5). Compounds (74-79) were active against Legionella pneumophila (Siddiqui et al., 2011).

Polyketide metabolites, 7,8-dihydonivefuranone A (80), 6(7)dehydro-8-hydroxyterrefuranone (81), 6-hydroxyterrefuranone (82) and Nivefuranones A (83) (Figure 6) were isolated from a Microdiplodia sp. KS 75-1 from the stems of conifer trees (Pinus sp.). Compounds (80-83) were active against S. aureus NBRC 13276 with zone of inhibition of $15,15,16$, and $15 \mathrm{~mm}$ respectively, tested at $40 \mu \mathrm{g} /$ per disc of $8 \mathrm{~mm}$ diameter (Shiono et al., 2012).

$1 \beta$-hydroxy- $\alpha$-cyperone (84) (Figure 6) was isolated from the endophyte Microsphaeropsis arundinis found in stems of Ulmus macrocarpa collected from Dongling Mountain, Beijing, People's Republic of China. Compound (84) inhibits S. aureus (CGMCC1.2465), at an MIC of $11.4 \mu \mathrm{g} / \mathrm{mL}$. Ampicillin (positive control) showed an MIC value of $0.46 \mu \mathrm{g} / \mathrm{mL}$ (Luo et al., 2013).

Microsphaeropsone A (85) and Microsphaeropsone C (86) (Figure 5), were isolated from Microsphaeropsis sp. (strain 8875) from the plant Lycium intricatum, co-occurs with their putative biogenetic Anthraquinoide precursors and Citreorosein (87). From a Microsphaeropsis species (strain no. 7177) of the plant Zygophyllum fortanesii from Gomera (Spain), large amounts of Fusidienol A (88) and the known aromatic xanthones (89), were isolated. The endophyte Seimatosporium species (internal strain no. 8883) of Salsola oppositifolia from Gomera (Spain), produced 3, 4-dihydroglobosuxanthone A (90). Compounds (85-90) were active against E. coli and B. megaterium (Krohn et al., 2009).

Dinemasones A(91) and B (92) (Figure 5), were isolated from Dinemasporium strigosum obtained from the roots of the herbaceous plant Calystegia sepium growing on the shores of the Baltic Sea, Wustrow, Germany. The above compounds showed antibacterial activities against B. megaterium (Krohn et al., 2008b).

Cytosporone D (93) and E (94) (Figure 7), were isolated from the endophyte CR200 (Cytospora sp.) and CR146 (Diaporthe sp.) present in tissues of Conocarpus erecta and Forsteronia spicata plants respectively collected in the Guanacaste Conservation Area of Costa Rica. Cytosporone D (93) shows antibacterial activity against $S$. aureus, E. faecalis, and E. coli with MICs of 8, 8, and $64 \mu \mathrm{g} / \mathrm{mL}$ respectively, while Cytosporones E (94) has similar activity against $S$. aureus (Brady et al., 2000).

Cytosporone D (93), E (94), and Cytoskyrin A (95) (Figure 7), were isolated from a Cytospora sp. CR200 from a branch of Conocarpus erecta (Buttonwood tree) in the Guanacaste National Park, from Costa Rica. Cytoskyrin A (95) has good in-vitro antibacterial activity (MICs against (S. aureus ATCC 29923, S. aureus ATCC6538P, S. aureus \#310 (MRSA), E. faecium \#379 (VREF), E. faecium \# 436 (VSEF), B. subtilis BGGS1A1, E. coli imp BAS849), ranging from 0.03 to $0.25 \mu \mathrm{g} / \mathrm{mL}$ ). Cytosporone $\mathrm{D}$ (93) and $\mathrm{E}$ (94) have moderate in-vitro antibacterial activity against above mentioned bacteria (MICs 8-64 $\mu \mathrm{g} / \mathrm{mL}$ ) (Singh et al., 2007).

Two new benzyl $\gamma$-butyrolactone analogs, (R)-5-((S)hydroxy(phenyl)-methyl)dihydrofuran-2(3H)-one (96) and its 6-acetate (97), a new naphthalenone derivative (98), together with aromatic derivatives, (S)-5-((S)-hydroxy(phenyl)-methyl) dihydrofuran-2(3H)-one (99), (S)-5-benzyl-dihydrofuran2(3H)-one (100), 5-phenyl-4-oxopentanoic acid (101), gamma-oxo-benzenepentanoic acid methyl ester (102), 3(2,5-dihydro-4-hydroxy-5-oxo-3-phenyl-2-furyl)propionic acid (103), (3R)-5-methylmellein (104), Integracins A (105), and B (106) (Figure 7) were isolated from Cytospora sp., of Ilex canariensis from Gomera. Compounds (96- 106) are active against $B$. megaterium, zone size range $15-25 \mathrm{~mm}$ when $50 \mu \mathrm{L}$ of a solution $(0.05 \mathrm{mg} / \mathrm{mL}$ substance) are pipetted onto $9 \mathrm{~mm}$ a sterile filter paper disc (Lu et al., 2011).

Chaetoglobosin B (107) (Figure 8), isolated from the endophyte Chaetomium globosum from the leaves of Viguiera robusta showed weak antibacterial activity against $S$. aureus (MIC $120 \mu \mathrm{g} / \mathrm{mL}$ ) and E. coli (MIC $189 \mu \mathrm{g} / \mathrm{mL}$ ) (Momesso et al., 2008).

Chaetoglocins A-B (108-109) (Figure 8) isolated from Chaetomium globosum strain IFB-E036, an endophyte from Cynodon doctylon have antimicrobial activity against B. subtilis, Streptococcus pyogens, Micrococcus luteus and Mycobacterium smegmatis with MICs between 8 and $32 \mu \mathrm{g} / \mathrm{mL}$ (Ge et al., 2011).

Antibacterial compounds Acremonisol A (110), Semicochliodinol A (111), Cochliodinol (112), were isolated 
<smiles>Cc1cc(O)c2c(c1)C(=O)[C@@H]1C=C(O)[C@@H](O)[C@H](O)[C@H]1[C@H]2O</smiles>

Coniothyrinone D (65)<smiles>O=C(O)CCO</smiles>

3-Hydroxypropionic Acid (66)<smiles>CCCCCc1c(O)c(OC)cc2c1[C@H](/C=C/C(=O)O)[C@@H](C)O2</smiles>

Botryomaman (67)<smiles>CCCCCc1cc(OC)cc(OC)c1O</smiles>

\section{2,4-dimethoxy-6} -pentylphenol (68)<smiles>[R2]C1([R2])c2cccc(O)c2C(=O)O[C@H]1C</smiles>

$\mathbf{R}_{\mathbf{1}}=\mathbf{R}_{\mathbf{2}}=\mathbf{H}(\mathbf{R})-(-)$-mellein (69)<smiles>CCCCCC1=CC(=O)C=C(OC)C1=O</smiles>

Primin (70)<smiles>CC(O)C(O)C=CC(=O)O</smiles>

4,5-dihydroxy-2-hexenoic acid (73)<smiles>[R]c1cc(O)c2c(c1)O[C@]1(C)[C@@H](O)CC[C@H](O)[C@]1(O)C2=O</smiles>

$\mathrm{R}=\mathrm{CH}_{3}$ Diversonol (74) $\mathrm{R}=\mathrm{CH}_{2} \mathrm{OH}$ Microdiplodiasol (75)<smiles>C[C@]1([C@H]2CCC(=O)O2)CC(=O)c2c(O)cc(O)cc2O1</smiles>

Microdiplodiasone (76)<smiles>COC1=CC(=O)[C@H](O)[C@@]2(O)C(=O)c3c(O)cc(C)cc3O[C@]12C</smiles>

Microdiplodiasolol (77)<smiles>C[C@@H]1Oc2cccc(O)c2C(=O)[C@H]1O</smiles>

(-)-Gynuraone (78)<smiles>CC(C)[C@H](C)/C=C/C(C)[C@]12CC[C@H]3[C@H]4CC=C5C[C@@H](O)CC[C@]5(C)[C@H]4CC[C@]31CC2</smiles>

Ergosterol (79)

FIGURE 5 | Structures of antibacterial metabolites isolated from Ascomycetes (65-79).

from C. globosum SNB-GTC2114 and Pyrrocidine A (113), $\mathrm{B}$ (114), C (115), and Alterperylenol (116) (Figure 8) were isolated from Lewia infectoria SNB-GTC2402 obtained from Besleria insolita from the Amazon Rainforest biome of Cayenne and Roura, French Guiana. Compounds (110-112, 115, and 116), exhibited antibacterial activity against $S$. aureus ATCC 29213 with MICs of 64, 2, 4, 2, and $32 \mu \mathrm{g} / \mathrm{mL}$ respectively. Compounds (113-114) were active against $S$. aureus ATCC 29213, with a MIC value of $5 \mu \mathrm{g} / \mathrm{mL}$ (Casella et al., 2013). 


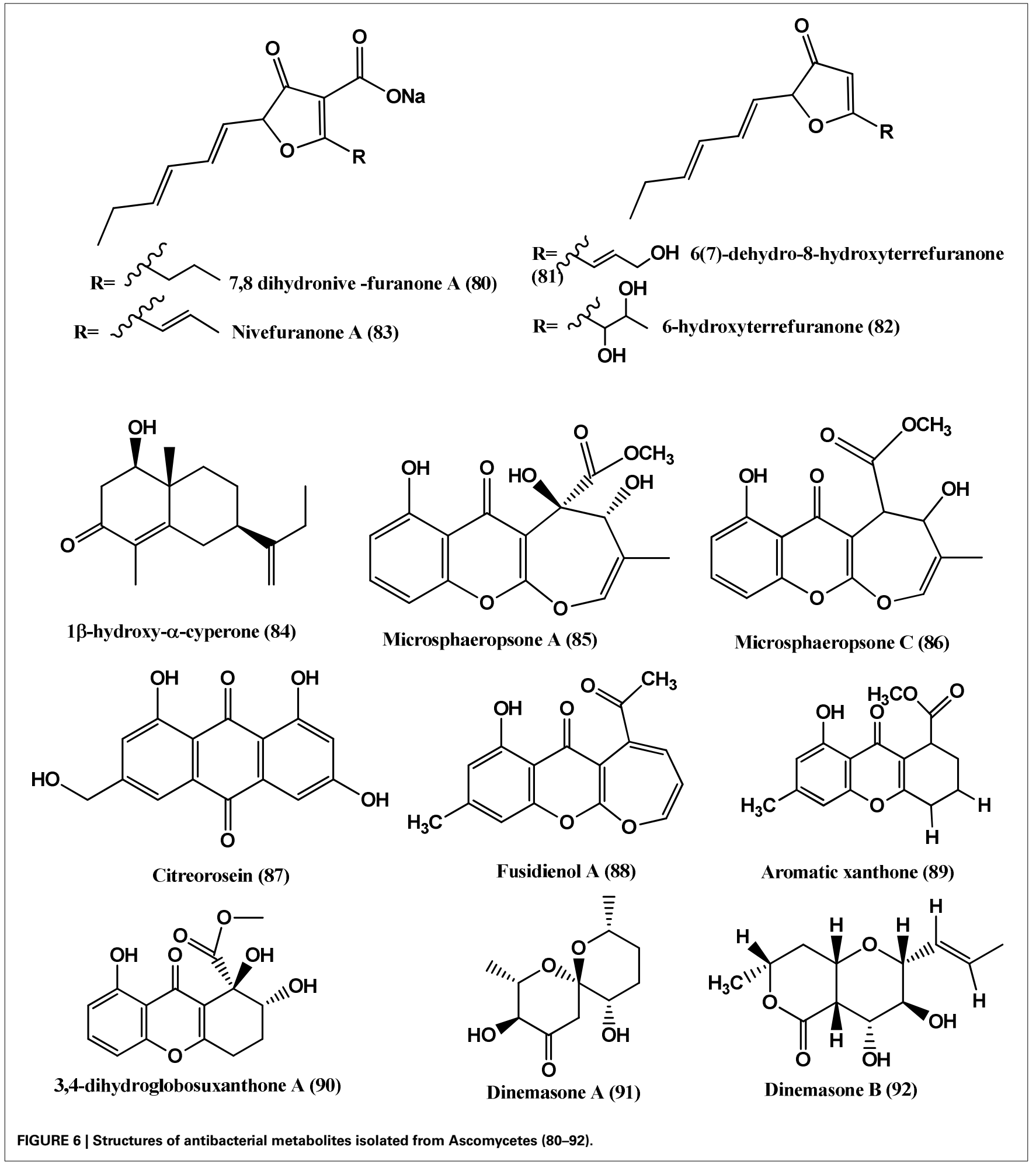

7-amino-4-methylcoumarin (117) (Figure 8) was isolated from the endophyte Xylaria sp., of Ginkgo biloba. The compound showed strong antibacterial against $S$. aureus, E. coli, S. typhi, Salmonella typhimurium, Salmonella enteritidis, Aeromonas hydrophila, Yersinia sp., Vibrio anguillarum, Shigella sp., and
Vibrio parahaemolyticus with MIC of $16,10,20,15,8.5$, $4,12.5,25,6.3$, and $12.5 \mu \mathrm{g} / \mathrm{mL}$ respectively (Liu et al., 2008).

1-(xylarenone A)xylariate A (118), Xylarioic acid B (119)

(Figure 8), Xylariolide A (120), Xylariolide B (121), Xylariolide 
<smiles>CCCCCCCC1OC(=O)Cc2cc(O)c(O)c(O)c21</smiles><smiles>[R][C@H]1c2ccccc2CC2CC(=O)O[C@H]21</smiles>

R=OH (R)-5-((S)-hydroxy(phenyl)-methyl) dihydrof uran-2(3H)-one (96) $R=O A c 6$ acetate of (R)-5-((S)-hydroxy (phenyl)-methyl)dihydrofuran-2(3H)-one (97)<smiles>[R]OC(=O)CCC(=O)Cc1ccccc1</smiles>

$R=H$ 5-phenyl-4-oxopentanoic acid (101)

$\mathrm{R}=\mathrm{Me} \mathrm{Y}$-oxo-benzenepentanoic acid methyl ester (102)<smiles>CCCCCCCC1OC(=O)c2cc(O)c(O)c(O)c21</smiles>

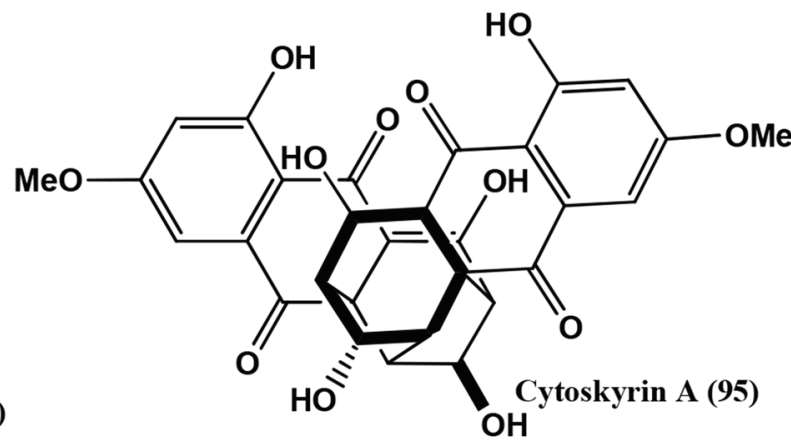<smiles>CC(=O)O[C@H]1CCC(=O)c2c(O)cccc21</smiles>

Naphthalenone derivative (98)<smiles>[R][C@H]1c2ccccc2CC2CC(=O)O[C@H]21</smiles>

R=OH (S)-5-((S)hydroxy(phenyl)methyl)dihydrofuran-2(3H)-one (99) $\mathrm{R}=\mathrm{H}$ (S)-5-benzyl-dihydrofuran-2(3H)one (100)<smiles>O=C(O)CCC1OC(=O)C(O)=C1c1ccccc1</smiles>

3-(2,5-dihydro-4-hydroxy-5oxo-3-phenyl-2-furyl)propionic acid (103)<smiles>Cc1ccc(O)c2c1C[C@H](C)OC2=O</smiles>

(3R)-5-methylmellein (104)

$R=A c$ Integracin $A(\mathbf{1 0 5})$<smiles>CCCCc1cc(O)cc(O)c1</smiles>

FIGURE 7 | Structures of antibacterial metabolites isolated from Ascomycetes (93-106).

C (122), Me-xylariate C (123), Xylariolide D (124), and taiwapyrone (125) (Figure 9), were isolated from Xylaria sp. NCY2 of Torreya jackii Chun collected from Jiangshi Nature Reserve Zone of Fujian Province, China. Compounds (118-125) are active against E. coli ATCC 25922, B. subtilis ATCC 9372 and
S. aureus ATCC 25923 with MIC values above $10 \mu \mathrm{g} / \mathrm{mL}$ (Hu et al., 2010).

The polyketide, Cryptosporioptide (126) (Figure 9) was isolated from a Cryptosporiopsis sp., from the shoot tissues of the shrub Viburnum tinus, collected from Gomera. At $50 \mu \mathrm{g}$ per 


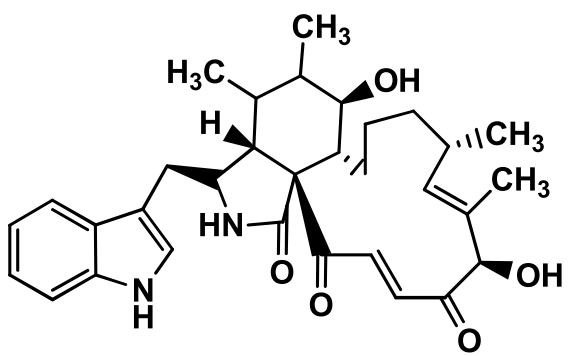

Chaetoglobosin B (107)<smiles>CCCc1c(Cl)c(OC)cc(OC)c1C(=O)O</smiles>

Acremonisol A (110)

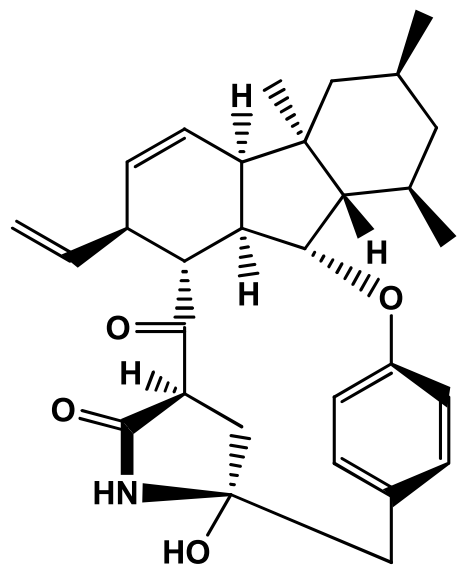

Pyrrocidine B (114)<smiles>C/C=C(/CO)c1oc(=O)cc(OC)c1CO</smiles>

Chaetoglocin A (108)<smiles>[R]c1ccc2[nH]cc(C3=C(O)C(=O)C(c4c[nH]c5ccc(CC=C(C)C)cc45)=C(O)C3=O)c2c1</smiles>

$\mathrm{R}=\mathrm{H}$, Semicochliodinol A (111) $\mathrm{R}=$ Prenyl, Cochliodinol (112)

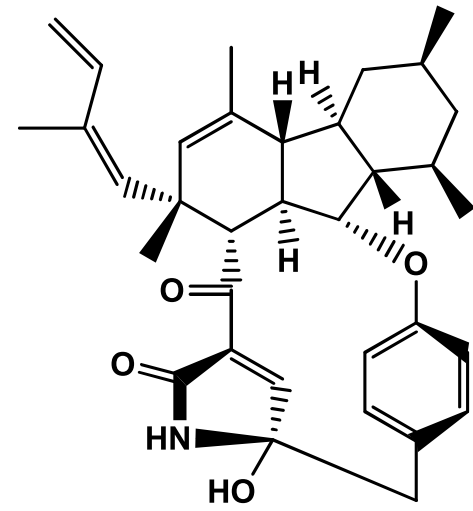

Pyrrocidine C (115)<smiles>CO/C=C(/CO)c1oc(=O)cc(OC)c1CO</smiles>

Chaetoglocin B (109)

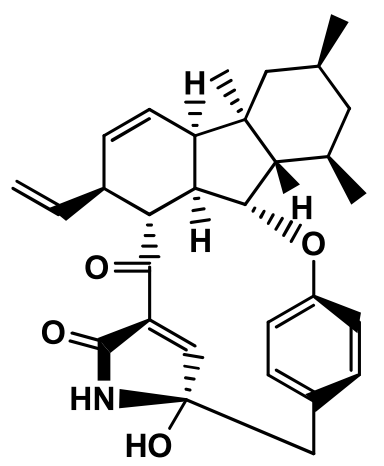

Pyrrocidine A (113)<smiles>O=C1CC[C@@]2(O)c3c(ccc(O)c31)-c1ccc(O)c3c1[C@@]2(O)[C@H](O)CC3=O</smiles><smiles>Cc1cc(=O)oc2cc(N)ccc12</smiles>

7-amino-4-methylcoumarin (117)<smiles>C=C(CO)[C@]1(O)C[C@]2(C)[C@@H](C)CC[C@@H](O)[C@H]2C=C1C(=O)C(C)C(O)/C(C)=C/C(C)CC</smiles>

1-(xylarenoneA)xylariate A (118)<smiles>CCC(C)C(O)C(C)(O)C(O)C(C)C=O</smiles>

Xylarioic acid B (119)

FIGURE 8 | Structures of antibacterial metabolites isolated from Ascomycetes (107-119). 
<smiles>CCC(C)C(O)[C@]1(C)OC(=O)[C@H](C)[C@@H]1O</smiles>

Xylariolide A (120)<smiles>CCC(C)(O)C(O)[C@]1(C)OC(=O)[C@H](C)[C@@H]1O</smiles>

Xylariolide B (121)<smiles>CC(O)C(C)C(O)[C@]1(C)OC(=O)[C@H](C)[C@@H]1O</smiles>

Xylariolide C (122)<smiles>CCC/C=C(/C=CC(=O)OC)C(C)=O</smiles>

Methyl xylariate C (123)

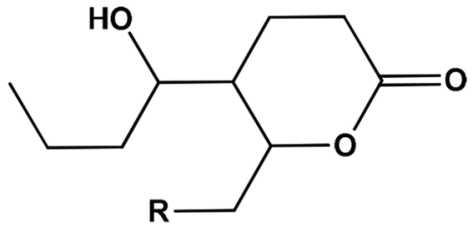

$\mathrm{R}=\mathrm{H}$ Xylariolide (124)

$\mathrm{R}=\mathrm{OH}$ Taiwapyrone (125)<smiles>[R]B(CCC)C[C@H]1OC(=O)c2c(cc(OC)c(OC)c2O)[C@@H]1O</smiles>

$\mathrm{R}=\alpha-\mathrm{OH}$ Isocoumarin $(129)$<smiles>COC(=O)[C@]12Cc3ccc(-c4ccc5c(c4O)C(=O)C4=C(O)C[C@@H](C)[C@H](O)[C@]4(C(C)=O)O5)c(O)c3C(=O)C1=C(O)C[C@H](C)[C@H]2O</smiles>

Secalonic Acid B (134)<smiles>O=C1C=CC(=O)C12C=CC1(Oc3cccc4cccc(c34)C1O)O2</smiles>

Spiropreussione A (137)

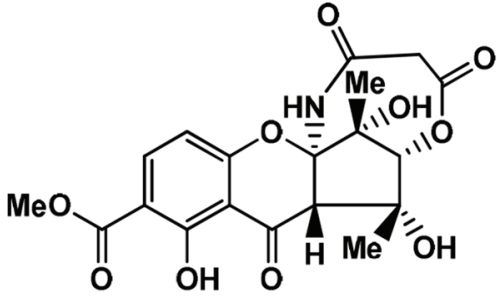

Cryptosporioptide (126)<smiles>[R]C(C)C[C@@H]1C[C@H]2OC(=O)c3c(cc(OC)c(OC)c3O)[C@@]2(C)O1</smiles>

$\mathrm{R}=\mathrm{H}$ Monocerin (127)

$\mathrm{R}=\alpha \mathrm{OH}(12 \mathrm{R})-12$-hydroxymonocerin (128)<smiles>[R]Cc1cc(O)c2c(=O)c3c(oc2c1)C=COC=C3C(=O)OC</smiles>

$R=H$ Isof usidienol A (130)
$R=$ OH Isofusidienol B (131)

$\mathrm{R}=\mathrm{H}$ Isofusidienol C (132) $\mathrm{R}=\mathrm{OH}$ Isof usidienol D (133)<smiles>[R]Cc1cc(O)c2c(=O)c3c(oc2c1)CCOC=C3C(=O)OC</smiles><smiles>COC(=O)[C@@]12Oc3cccc(O)c3C(=O)C1=C(O)C[C@@H](C)[C@H]2O</smiles><smiles>COC(=O)[C@]12Oc3ccccc3C(=O)C1=C(O)C[C@@H](C)[C@H]2O</smiles>

Blennolide A (135)

Blennolide B (136)<smiles>COc1cc(C)cc(O)c1C(=O)c1c(OC)cc(O)cc1OC</smiles>

Monomethylsulochrin (138)<smiles>COc1cc(C)cc(O)c1C(=O)c1c(OC)cc(O)cc1C(=O)O</smiles>

Rhizoctonic Acid (139)

FIGURE 9 | Structures of antibacterial metabolites isolated from Ascomycetes (120-139). 
$9 \mathrm{~mm}$ paper disc, it inhibits $B$. megaterium, showing a $9 \mathrm{~mm}$ radius of zone of inhibition (Saleem et al., 2013).

Monocerin (127), (12S)-12-hydroxymonocerin (128) and Isocoumarin (129) (Figure 9) were isolated from Microdochium bolleyi, an endophyte from Fagonia cretica. All these compounds were active against E. coli and B. megaterium (Zhang et al., 2008a).

Isofusidienol A (130), B (131), C (132), and D (133) (Figure 9) were isolated from a Chalara sp. strain 6661, an endophyte of Artemisia vulgaris, collected from Ahrenshoop, Germany Compounds (130) and (131) showed strong antibacterial activities against $B$. subtilis with inhibition zones of 23 and $22 \mathrm{~mm}$ respectively, at $15 \mu \mathrm{g}$ of compounds per 6-mm filter disks. Under the same conditions, $15 \mu \mathrm{g}$ of Penicillin $\mathrm{G}$ has a zone of $50-\mathrm{mm}$ diameter. The MIC of compound (130) was shown to be $0.625 \mu \mathrm{g}$ on 6-mm filter disks. Compound (130) shows moderate activity against $S$. aureus and $E$. coli with an inhibition zone diameter of 9 and $8 \mathrm{~mm}$, respectively, at $15 \mu \mathrm{g}$ of compound per 6-mm filter disk. Compound (132) and (133) show inhibition zone of 9 and $8 \mathrm{~mm}$ against $B$. subtilis at $15 \mu \mathrm{g}$ per 6-mm filter disk (Loesgen et al., 2008).

Secalonic acid B (134), Blennolides A (135) and B (136) (Figure 9) were isolated from a Blennoria sp., an endophyte of Carpobrotus edulis, from El Cedro, Gomera. Compounds (134136) inhibit B. megaterium, and compounds (135) and (136) also inhibited E. coli (Zhang et al., 2008b).

Spiropreussione A (137) (Figure 9) was obtained from an endophyte, Preussia sp., of the mature stems of Aquilaria sinensis (Thymelaeaceae), collected from the Guangxi Medicinal Arboretum. Spiropreussione A (137) shows activity against S. aureus (CMCC B26003) with a zone of inhibition of $16.4 \pm$ $0.3 \mathrm{~mm}(n=3)$ at $5 \mu \mathrm{g} / \mathrm{disk}$. The MIC of the compound in agar dilution test using NCCLS 2002 guide lines was $25 \mu \mathrm{M}$ (Chen et al., 2009).

Monomethylsulochrin (138), Rhizoctonic acid (139), (Figure 9) and Guignasulfide (140) (Figure 10) were isolated from a Guignardia sp. IFB-E028, an endophyte of Hopea hainanensis and show moderate activity against the human bacterial pathogen Helicobacter pylori with MIC values of 28.9, 60.2, and $42.9 \mu \mathrm{M}$, respectively (Wang et al., 2010).

Helvolic acid (141) (Figure 10) was isolated from the endophyte Pichia guilliermondii Ppf9 of medicinal plant Paris polyphylla var. yunnanensis. Compound (141) has strongest antibacterial activity on Agrobacterium tumefaciens, E. coli, Pseudomonas lachrymans, Ralstonia solanacearum, Xanthomonas vesicatoria, B. subtilis, S. aureus, and Staphylococcus haemolyticus, with MICs of $1.56,3.13,3.13,1.56,1.56,3.13,50$, and $6.25 \mu \mathrm{g} / \mathrm{mL}$, respectively (Zhao et al., 2010).

Chlorogenic acid (142) (Figure 10) was isolated from the endophyte strain B5 a Sordariomycete sp. of Eucommia ulmoides. Eucommia ulmoides is a medicinal plant of China and one of the main sources of Chlorogenic acid. It has antibacterial, antifungal, antioxidant and antitumor activities (Chen et al., 2010).

Antibacterial Biscogniazaphilones A (143) and B (144), N-trans-feruloy-3-O-methyldopamine (145), 5-Hydroxy-3,7,4trimethoxyflavone (146), 4-Methoxycinnamaldehyde (147), Methyl 3,4-methylenedioxycinnamate (148), 4-Methoxytrans-cinnamic acid (149), (Figure 10) were isolated from the endophyte Biscogniauxia formosana BCRC 33718, of Cinnamomum sp. Compounds (143) and (144) show antimycobacterial activities against $M$. tuberculosis strain $\mathrm{H} 37 \mathrm{Rv}$ in vitro showing MIC values of $\leq 5.12$ and $\leq 2.52 \mu \mathrm{g} / \mathrm{mL}$, respectively, than the clinical drug Ethambutol (MIC $6.25 \mu \mathrm{g} / \mathrm{mL}$ ). Compounds (145-149) show moderate to weak antimycobacterial activities with MICs of 12.5, 25.0, 42.1, 58.2, and $50.0 \mu \mathrm{g} / \mathrm{mL}$, respectively (Cheng et al., 2012).

Dothideomycetide A (150) (Figure 10) from an endophyte a Dothideomycete sp., of a Thai medicinal plant, Tiliacora triandra, has antibacterial activity against $S$. aureus ATCC 25923 and MRSA ATCC 33591 with MIC values of 128 and $256 \mu \mathrm{g} / \mathrm{mL}$ respectively (Senadeera et al., 2012).

Cristatumins A (151) and Tardioxopiperazine A (152) (Figure 10) were produced by the endophyte Eurotium cristatum EN-220 of marine alga Sargassum thunbergii and showed activity against E. coli and S. aureus with MIC values of 64 and $8 \mu \mathrm{g} / \mathrm{mL}$, respectively (Du et al., 2012).

\section{COMPOUNDS PRODUCED BY HYPHOMYCETES}

Hyphomycete form a class of fungi which produces the asexual spores. Producers of the antibacterials Penicillins and Cephalosporins belong to this class. Other antibacterials from this class are Helvolic acid (141) (Figure 10), Monomethylsulochrin (138) (Figure 9), Ergosterol (79) (Figure 5) and 3 $\beta$-Hydroxy-5 $\alpha$, $8 \alpha$-epidioxy-ergosta-6, 22-diene (153) (Figure 11) were isolated from an endophyte Aspergillus sp. CY725 of Cynodon dactylon (Poaceae). Compounds (141), (138), (79), and (153) are active against $H$. pylori with MICs of $8.0,10.0,20.0$, and $30.0 \mu \mathrm{g} / \mathrm{mL}$ respectively. Helvolic acid (141) is active against Sarcina lutea and S. aureus with MICs of 15.0 and $20.0 \mu \mathrm{g} / \mathrm{mL}$ respectively (Li et al., 2005).

Aspergicin (154) and Neoaspergillic acid (155) (Figure 11) were isolated from a mixture of cultured mycelia of two marinederived mangrove epiphytic Aspergilli FSY-01 and FSW-02. Aspergicin (154) has anti-bacterial activity against $S$. aureus, S. epidermidis, B. subtilis, B. dysenteriae, B. proteus, and E. coli, with MICs of $62.5,31.2515 .62,15.6262 .5$, and $31.25 \mu \mathrm{g} / \mathrm{mL}$ respectively. Neoaspergillic acid (155) has antibacterial activity against $S$. aureus, S. epidermidis, B. subtilis, B. dysenteriae, B. proteus, and E. coli, with MICs of $0.98,0.49,1.95,7.8,7.8$, and $15.62 \mu \mathrm{g} / \mathrm{mL}$ respectively (Zhu et al., 2011).

Two new dihydroisocoumarin derivatives Aspergillumarins A (156) and B (157) (Figure 11) are produced by a marine-derived Aspergillus sp., of the mangrove Bruguiera gymnorrhiza collected from the South China Sea. Both show weak antibacterial activities against $S$. aureus and B. subtilis at $50 \mu \mathrm{g} / \mathrm{mL}$ (Li et al., 2012).

Brevianamide M (158), 6, 8-di-O-methylaverufin (159) and 6-O-Methylaverufin (160) (Figure 11), were isolated from Aspergillus versicolor a fungus of the marine brown alga Sargassum thunbergii. These compounds have activities against $S$. aureus and E. coli (Miao et al., 2012).

Isorhodoptilometrin-1-Me ether (161), Siderin (162) (Figure 11), were isolated from the marine fungus Aspergillus versicolor of inner tissues of the Red Sea green alga Halimeda opuntia. Both the compounds show moderate activity against 


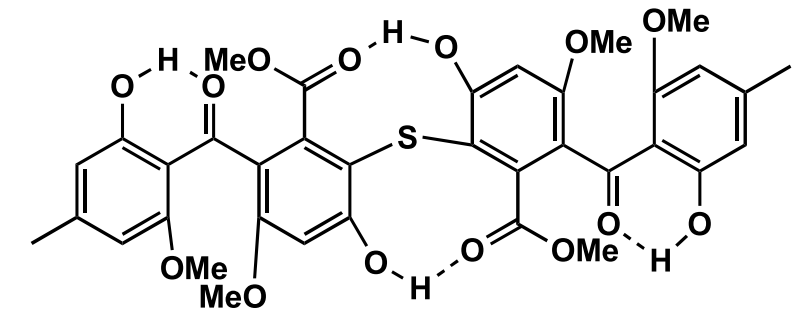

Guignasulfide (140)

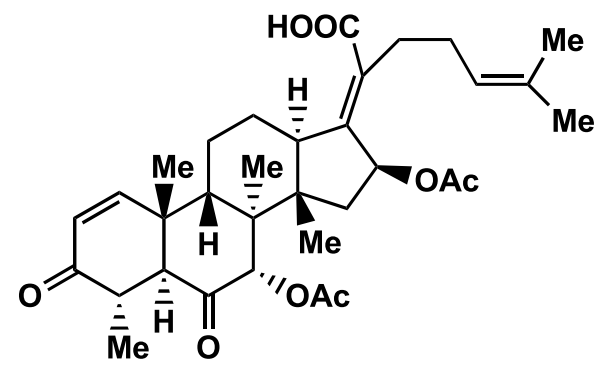<smiles>O=C(/C=C/c1ccc(O)c(O)c1)O[C@H]1C[C@@](O)(C(=O)O)C[C@@H](O)[C@H]1O</smiles>

Chlorogenic Acid (142)<smiles>C/C=C/C1=CC2=CC(=O)[C@](C)(O)[C@H](CC(=O)CCCCCCCCCC)C2=CO1</smiles>

Biscogniazaphilones A (143)

Helvolic Acid (141)<smiles>C/C=C/C1=CC2=CC(=O)[C@]3(C)OC(=O)[C@H](C(=O)CCCCCCCCC)[C@H]3C2=CO1</smiles>

Biscogniazaphilones B (144)<smiles>COc1cc(/C=C/C(=O)NCCc2ccc(O)c(OC)c2)ccc1O</smiles>

N-trans-feruloy-3-O-methyldopamine (145)<smiles>COc1ccc(-c2oc3cc(OC)cc(O)c3c(=O)c2OC)cc1</smiles>

5-hydroxy-3,7,4'-trimethoxyflavone

4-methoxycinnamaldehyde (147)<smiles>COC(=O)/C(C)=C/c1ccc2c(c1)OCO2</smiles><smiles>COc1ccc(/C=C/C(=O)O)cc1</smiles>

Methyl 3,4-methylenedioxycinnamate (148)

$$
\text { 4-methoxy-trans-cinnamic acid (149) }
$$<smiles>CC[C@H](C)[C@H](O)[C@H](C)C(=O)O[C@]1(C)C(=O)c2c(cc(O)c3c2CCC[C@@H]3C)C(C)(O)C1=O</smiles>

Dothideomycetide A (150)<smiles>C=CC(C)(C)c1[nH]c2ccccc2c1/C=C1\NC(=O)[C@@H](CO)NC1=O</smiles><smiles>[R]c1ccc2[nH]c(C(C)(C)C=C)c(C[C@@H]3NC(=O)[C@H](C)NC3=O)c2c1</smiles>

Cristatumin A (151)<smiles>COc1ccc(/C=C/C=O)cc1</smiles> 
<smiles>CC(C)C(C)/C=C/C(C)C1CCC2C1(C)CCC1C23C=CC12OOC2(C)CCC(O)C3</smiles><smiles>COc1cc2c3c(c1)c(=O)nc([C@@H]1CCCN1)n3-c1ccccc1C(=O)O2</smiles>

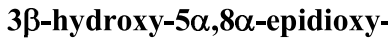
ergosta-6,22-diene (153)<smiles>CC(=O)CCC[C@H]1Cc2cccc(O)c2C(=O)O1</smiles>

Aspergillumarin A (156)
Aspergicin (154)<smiles>CC(O)CCC[C@H]1Cc2cccc(O)c2C(=O)O1</smiles>

Aspergillumarin B (157)<smiles></smiles>

Neoaspergillic acid(155)<smiles>O=C1N[C@@H](O)c2nc3ccccc3c(=O)n2[C@H]1Cc1ccccc1</smiles>

Brevianamide M (158)<smiles>COc1cc(OC)c2c(c1)C(=O)c1cc3c(c(O)c1C2=O)[C@H]1CCC[C@](C)(O3)O1</smiles>

6,8-di-O-methylaverufin (159)<smiles>COc1cc(O)c2c(c1)C(=O)c1cc3c(c(O)c1C2=O)[C@H]1CCC[C@](C)(O3)O1</smiles><smiles>COc1cc(CC(C)O)cc2c1C(=O)c1c(O)cc(O)cc1C2=O</smiles>

6-O-methylaverufin (160)
Isorhodoptilometrin-1-methyl ether (161)<smiles>COc1cc(C)c2c(OC)cc(=O)oc2c1</smiles>

Siderin (162)<smiles>[R]OC(=O)c1cc(OC)c2c(=O)c3c(O)cc(C)cc3oc2c1</smiles>

$\mathrm{R}=\mathrm{CH}_{3}$ Yicathin $\mathrm{B}(\mathbf{1 6 3})$

$\mathrm{R}=\mathrm{H}$ Yicathin $\mathrm{C}$ (164)<smiles>C=CC(C)(C)c1[nH]c2cccc3c2c1C[C@@H]1[C@@H]3[C@H](OC(C)=O)[C@@H](C)CN1C</smiles>

Fumigaclavine $\mathrm{C}$ (165)

FIGURE 11 | Structures of antibacterial metabolites isolated from Hyphomycetes (153-165).

Bacillus cereus, B. subtilis, and S. aureus at a concentration of $50 \mu \mathrm{g} / \mathrm{disc}$ of $9 \mathrm{~mm}$ (Hawas et al., 2012).

Yicathin B (163) and C (164) (Figure 11) were isolated from the endophyte Aspergillus wentii PT-1 of the red marine alga Gymnogongrus flabelliformis. Tested in the agar diffusion assay at
$10 \mathrm{mg} /$ disk compound (163) was active against $E$. coli (inhibition zone diameter $9 \mathrm{~mm}$ ) and (164) a zone diameter of $12.0 \mathrm{~mm}$ and against S. aureus $7.5 \mathrm{~mm}$ (Sun et al., 2013b).

The alkaloids, Fumigaclavine C (165) (Figure 11) and Pseurotin A (166) (Figure 12) were isolated from the endophyte 
<smiles>CC/C=C\[C@H](O)[C@@H](O)C1=C(C)C(=O)[C@@]2(O1)C(=O)N[C@](OC)(C(=O)c1ccccc1)C[C@H]2O</smiles>

Pseurotin A (166)<smiles>Cc1cc(O)c2c(c1)C(=O)c1cc(O)cc(O)c1C2=O</smiles>

Emodin (168)

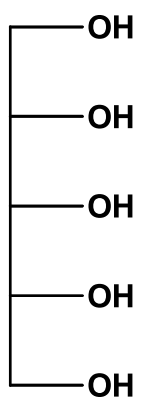

Erythritol (169)<smiles>CC[C@H](C)/C=C(C)/C=C/C1=CC2=C(Cl)C(=O)[C@](C)(OC(C)=O)C(=O)C2=CO1</smiles>

Sclerotiorin A (167)

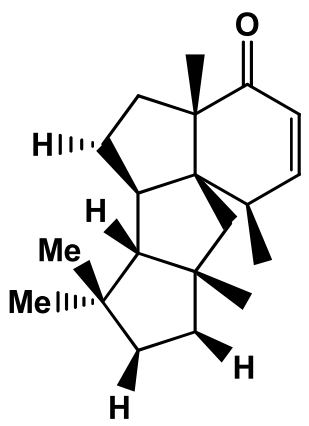

Conidiogenone B (170)

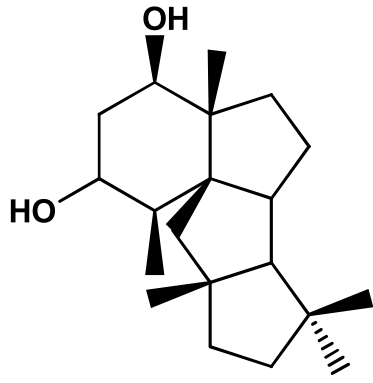

Conidiogenol (171)<smiles>C=CC(C)(C)c1[nH]c2ccccc2c1C=C1NC(=O)C(C)(C)NC1=O</smiles>

3,1'-didehydro-3[2"(3'",3'"-dimethylprop-2-enyl)-3"-indolylmethylene]-6methyl pipera-zine-2,5-dione) (172)

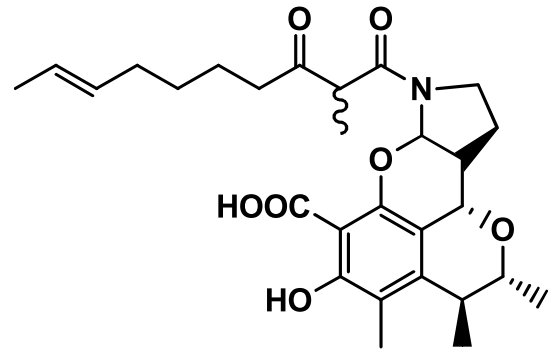

Perinadine A (173)<smiles>Cc1cc(O)cc2oc(=O)c3c(O)cc(O)cc3c12</smiles>

Alternariol (174)<smiles>CC1=C2C(=CO[C@H](C)[C@@H]2C)C(O)=C(C(=O)O)C1=O</smiles>

Citrinin (175)

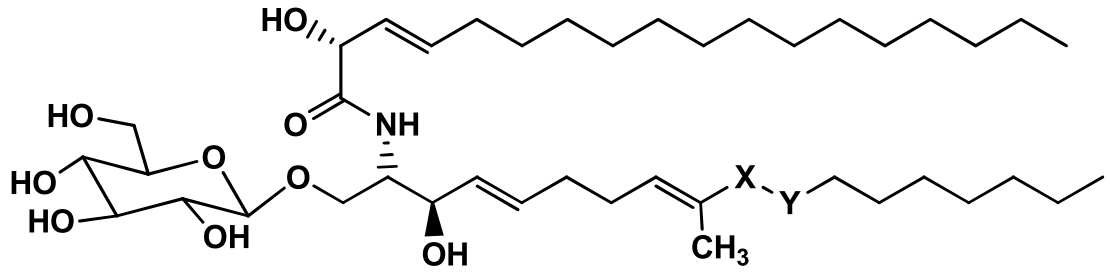

$\mathrm{X}-\mathrm{Y}=\mathrm{CH}_{2} \mathrm{CH}_{2},\left(2 \mathrm{~S}, 2^{\prime} \mathrm{R}, 3 \mathrm{R}, 3^{\prime} \mathrm{E}, 4 \mathrm{E}, 8 \mathrm{E}, 10 \mathrm{E}\right)-1-\mathrm{O}$-beta-D-glucopyranosyl-

2-N-(2'-hydroxy-3'-octadecenoyl)-3-hydroxy-9-methyl-4,8,10-sphingatrienine

(176)

$\mathrm{X}-\mathrm{Y}=\mathbf{C H}=\mathbf{C H},\left(2 \mathrm{~S}, \mathbf{2}^{\prime} \mathrm{R}, 3 \mathrm{R}, 3^{\prime} \mathbf{E}, 4 \mathrm{E}, \mathbf{8 E}\right)-1-\mathrm{O}-$ beta-D-glucopyranosyl-2-N-

(2'-hydroxy-3'-octadecenoyl)-3-hydroxy-9-methyl-4,8-sphingadienine (177)

FIGURE 12 | Structures of antibacterial metabolites isolated from Hyphomycetes (166-177). 
Aspergillus sp. EJC08, of the medical plant Bauhinia guianensis. Fumigaclavine $C$ (165) has activity against B. subtilis, E. coli, $P$. aeruginosa, and $S$. aureus with MICs of 7.81, 62.50, 31.25, and $15.62 \mu \mathrm{g} / \mathrm{mL}$ respectively, while Pseurotin A (166) has activity against $B$. subtilis, E. coli, P. aeruginosa, and S. aureus with MICs of $15.62,31.25,31.25$, and $15.62 \mu \mathrm{g} / \mathrm{mL}$ respectively (Pinheiro et al., 2013).

Pseurotin A (166) (Figure 12) was isolated from Penicillium janczewskii of the Chilean gymnosperm Prumnopitys andina. The compound shows moderate activity against phytopathogenic bacteria Erwinia carotovora and Pseudomonas syringae, with $\mathrm{IC}_{50}$ values of 220 and $112 \mu \mathrm{g} / \mathrm{mL}$, respectively (Schmeda-Hirschmann et al., 2008).

(+)-Sclerotiorin (167) (Figure 12), was isolated from the endophyte Penicillium sclerotiorum PSU-A13 (Arunpanichlert et al., 2010). Compound (167) has been reported to have antibacterial activity against S. aureus ATCC 29213 (MIC $128 \mu \mathrm{g} / \mathrm{mL}$ ) (Lucas et al., 2007).

Emodin (168) and Erythritol (169) (Figure 12) were isolated from the endophyte Penicillium citrinum strain ZD6 of the stems of Bruguiera gymnorrhiza. Emodin (168) and Erythritol (169) inhibit the growth of $B$. subtilis with MIC values of $25 \mu \mathrm{g} / \mathrm{mL}$ and $50 \mu \mathrm{g} / \mathrm{mL}$ respectively, while Emodin (168) was weakly active against $P$. aeruginosa at an MIC value of $100 \mu \mathrm{g} / \mathrm{mL}$ (Li et al., 2010).

Antibacterial Conidiogenone B (170) and Conidiogenol (171) (Figure 12) were isolated from Penicillium chrysogenum QEN$24 \mathrm{~S}$, an endophyte of a marine red algal species of the genus Laurencia. Conidiogenone B (170) has potent activity against MRSA, Pseudomonas fluorescens, P. aeruginosa, and S. epidermidis (at a concentration of $8 \mu \mathrm{g} / \mathrm{mL}$ ), while Conidiogenol (171) is activity against $P$. fluorescens and $S$. epidermidis (both at an MIC value of $16 \mu \mathrm{g} / \mathrm{mL}$ ) (Gao et al., 2011).

(3, $1^{\prime}$-didehydro-3[2" $\quad\left(3^{\prime \prime \prime}, \quad 3^{\prime \prime \prime}\right.$-dimethyl-prop-2-enyl)$3^{\prime \prime}$-indolylmethylene]-6-Mepipera-zine-2, 5-dione) (172) (Figure 12) was isolated from Penicillium chrysogenum MTCC 5108, an endophyte of the mangrove plant Porteresia coarctata (Roxb.), which has significant activity against Vibrio cholera MCM B-322 (Devi et al., 2012).

Perinadine A (173), Alternariol (174), and Citrinin (175) (Figure 12) were isolated from Penicillium citrinum present on the flowers of Ocimum tenuiflorum (Lamiaceae) collected in Denpasar, Bali, Indonesia. Compounds (173-175) were moderately active against S. aureus ATCC 29213 (MICs $64 \mu \mathrm{g} / \mathrm{mL}$ ). These compounds, failed to inhibit the E. coli ATCC 25922, and $P$. aeruginosa B 63230 at $64 \mu \mathrm{g} / \mathrm{mL}$ (Lai et al., 2013).

Fusarusides ( $\left.2 \mathrm{~S}, 2^{\prime} \mathrm{R}, 3 \mathrm{R}, 3^{\prime} \mathrm{E}, 4 \mathrm{E}, 8 \mathrm{E}, 10 \mathrm{E}\right)-1-\mathrm{O}-\beta$-D-glucopy-ran osyl-2-N-(2' -hydroxy-3' -octadecenoyl)-3-hydroxy-9-methyl-4,8, 10-sphingatrienine (176), $\left(2 \mathrm{~S}, 2^{\prime} \mathrm{R}, 3 \mathrm{R}, 3^{\prime} \mathrm{E}, 4 \mathrm{E}, 8 \mathrm{E}\right)-1-\mathrm{O}-\beta-\mathrm{D}-$ glucopyranosyl-2-N-(2'-hydroxy-3' -octadecenoyl)-3-hydroxy-9methyl-4,8-sphingadienine (177) (Figure 12) were isolated from a Fusarium sp. IFB-121, an endophyte of Quercus variabilis. Both cerebrosides have strong antibacterial activities against B. subtilis, E. coli and P. fluorescens with MIC values of 3.9, 3.9 and $1.9 \mu \mathrm{g} / \mathrm{mL}$ and $7.8,3.9$, and $7.8 \mu \mathrm{g} / \mathrm{mL}$ respectively (Shu et al., 2004).
Fusapyridon A (178) (Figure 13) was isolated from Fusarium sp. YG-45, an endophyte of the stem of Maackia chinensis, collected at Gottingen (Germany). The compound is active against $P$. aeruginosa and S. aureus, with MIC values of 6.25 and $50 \mu \mathrm{g} / \mathrm{mL}$, respectively (Tsuchinari et al., 2007).

Beauvericin (179) (Figure 13) was found in the endophyte Fusarium redolens Dzf2, of the rhizomes of Dioscorea zingiberensis. The $\mathrm{IC}_{50}$ values of Beauvericin against six test bacteria viz. B. subtilis, Staphylococcus hemolyticus, Pseudomonas lachrymans, Agrobacterium tumefaciens, E. coli and X. vesicatoria were between 18.4 and $70.7 \mu \mathrm{g} / \mathrm{mL}$ (Xu et al., 2010b). Beauvercin and (-)-4, 6'-anhydro-oxysporidinone (180) (Figure 13) were isolated from the endophyte Fusarium oxysporum of the bark of Cinnamomum kanehirae from Jiaoban Mountain, Taiwan Province. Beauvericin (179) is active against MRSA and B. subtilis at MICs of $3.125 \mu \mathrm{g} / \mathrm{mL}$. (-)-4, $6^{\prime}$-anhydro-oxysporidinone (180) has weak anti-MRSA activity (MIC, $100 \mu \mathrm{g} / \mathrm{mL}$ ) and moderate activity against $B$. subtilis (MIC, $25 \mu \mathrm{g} / \mathrm{mL}$ ) (Wang et al., 2011).

Javanicin (181), 3-O-methylfusarubin (182), a diastereomer of Dihydronaphthalenone (183) and 5-Hydroxy-3methoxydihydrofusarubin A (184) (Figure 13) were isolated from the endophyte Fusarium sp. BCC14842 of Bamboo leaf, collected from the Bamboo forest at Nam Nao National Park, Phetchabun Province, Thailand. Compound (181), and (183) have moderate activities (MICs of $25 \mu \mathrm{g} / \mathrm{mL}$ ) while 3-O-methylfusarubin (182) and 5-hydroxy-3-methoxydihydrofusarubin A (184) showed weak antimycobacterial activity (MICs of $50 \mu \mathrm{g} / \mathrm{mL}$ ) (Kornsakulkarn et al., 2011).

Fusaric acid was obtained from a Fusarium sp. an endophyte of a mangrove plant. Cadmium and Copper metal complexes were prepared. The Cadmium (185) and Copper (186) (Figure 13) complexes of fusaric acid exhibited potent inhibitory activity against the Mycobacterium bovis BCG strain with MIC $4 \mu \mathrm{g} / \mathrm{mL}$ and the M. tuberculosis $\mathrm{H} 37 \mathrm{Rv}$ strain with MIC $10 \mu \mathrm{g} / \mathrm{mL}$ respectively (Pan et al., 2011).

Fumitremorgin B (187), Fumitremorgin C (188), Helvolic acid (141), Bisdethiobis (methylthio) gliotoxin (189) (Figure 13), Bis-N-norgliovietin (190) and Gliotoxin (191) (Figure 14) were isolated from the endophyte Fusarium solani of Ficus carica. All compounds are active against B. subtilis, S. aureus, and E. coli and $P$. aeruginosa with MICs in the range of $0.5-16 \mu \mathrm{g} / \mathrm{mL}$ (Zhang et al., 2012).

Lateropyrone (192), Enniatins B1 (193) and A1 (194) (Figure 14), were isolated from mix culture fermentation of the fungal endophyte Fusarium tricinctum and the bacterium B. subtilis 168 trpC2 on solid rice medium. Fusarium tricinctum was obtained from rhizomes of Aristolochia paucinervis of the mountains of Beni-Mellal, Morocco. Enniatins B1 (193) and A1 (194), inhibit the growth the B. subtilis strain (MICs of 16 and $8 \mu \mathrm{g} / \mathrm{mL}$, respectively) and were also active against $S$. aureus, $S$. pneumoniae, and E. faecalis with MIC values in the range $2-8 \mu \mathrm{g} / \mathrm{mL}$. Lateropyrone (192) has antibacterial activity against B. subtilis, $S$. aureus, $S$. pneumoniae and E. faecalis, with MICs values ranging from 2 to $8 \mu \mathrm{g} / \mathrm{mL}$. All the above compounds were equally effective against a multi-drug-resistant clinical isolate of $S$. aureus (Ola et al., 2013). 


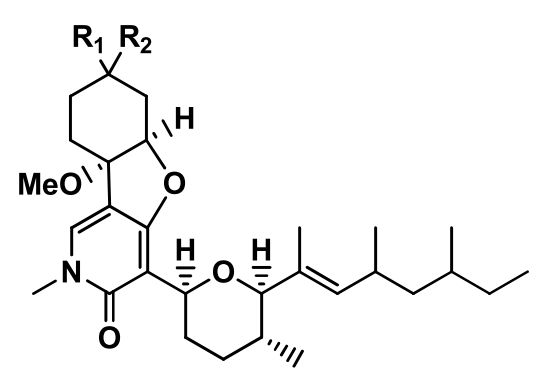

R1=R2=O, Fusapyridon A (178)

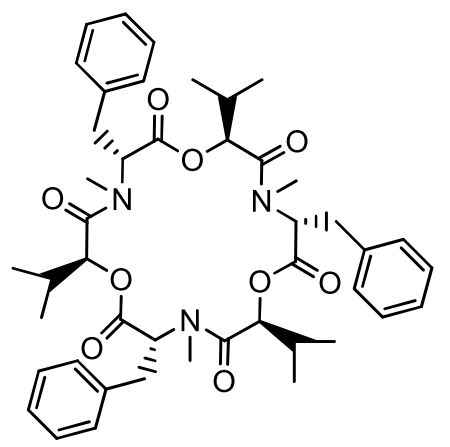<smiles></smiles>

-(-)-4,6'- anhydrooxysporidinone (180)
Beauvericin (179)<smiles>COC1=CC(=O)c2c(O)c(C)c(CC(C)=O)c(O)c2C1=O</smiles><smiles>COc1cc(O)c2c(c1O)[C@H]1O[C@H]2C(=O)[C@H]1CC(C)=O</smiles>

Javanicin (181)<smiles>COc1cc(O)c2c(c1O)C(=O)C1=C(COC(C)(OC)C1)C2=O</smiles><smiles>COc1cc(O)c2c(c1O)[C@H](O)[C@H]1C[C@](C)(OC)C[C@H]1C2=O</smiles>

5-hydroxy-3-methoxydihydrof usarubin A (184)

3-O-Methylf usarubin (182) Diastereomer of dihydronaphthalenone (183)

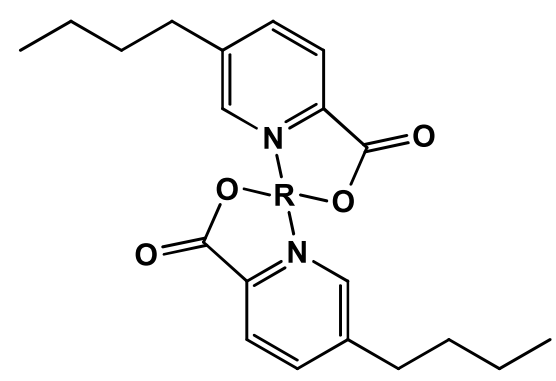

$\mathbf{R}=\mathbf{C d}$ (II), metal complex of Fusaric Acid (185)

$\mathrm{R}=\mathrm{Cu}$ (II), metal complex of Fusaric Acid(186)

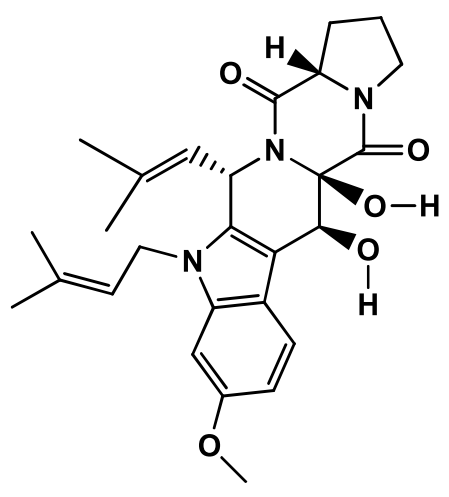

Fumitremorgin B (187)

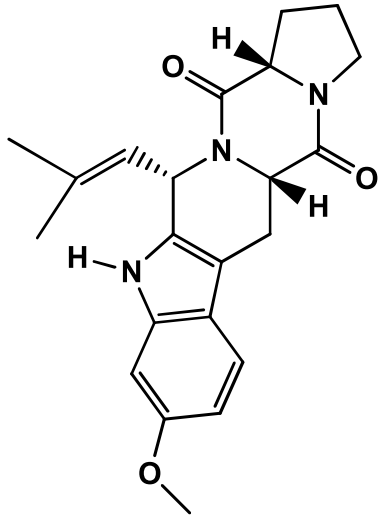

Fumitremorgin C (188)

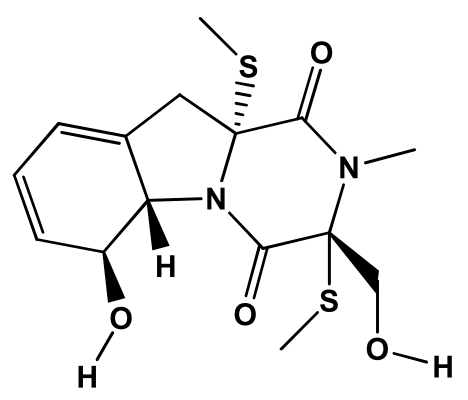

Bisdethiobis(methylthio) gliotoxin (189)

FIGURE 13 | Structures of antibacterial metabolites isolated from Hyphomycetes (178-189). 
<smiles>CS[C@]1(CO)C(=O)N(C)[C@]2(SC)CC3=C([C@H](O)CC=C3)N2C1=O</smiles>

Bis-N-norgliovietin (190)

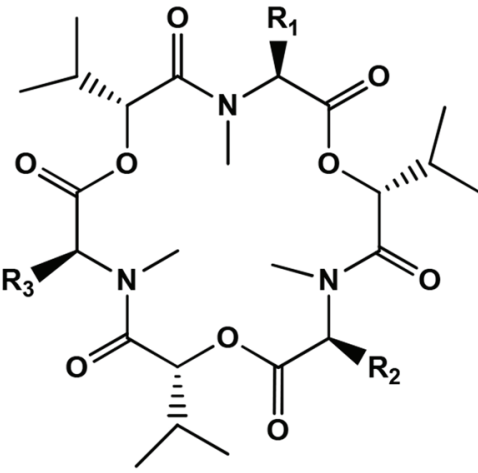

$\mathrm{R}_{\mathbf{1}}=\mathrm{R}_{2}=\mathrm{CH}\left(\mathrm{CH}_{3}\right)_{2}, \mathrm{R}_{3}=\mathrm{CH}\left(\mathrm{CH}_{3}\right) \mathrm{CH}_{2} \mathrm{CH}_{3}$ Enniatin B1(193)

$\mathrm{R}_{1}=\mathrm{R}_{2}=\mathrm{CH}\left(\mathrm{CH}_{3}\right) \mathrm{CH}_{2} \mathrm{CH}_{3}, \mathrm{R}_{3}=\mathrm{CH}\left(\mathrm{CH}_{3}\right)_{2}$ Enniatin A1(194)

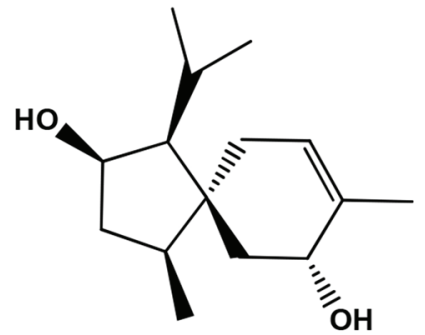

2ß-hydroxytrichoacorenol (199)<smiles>CN1C(=O)C2(Cc3ccccc3)NC(=O)C1(CO)SS2</smiles>

Gliotoxin (191)<smiles>O=C(O)c1cc(O)c2c(c1)C(=O)c1cccc(O)c1C2=O</smiles>

Rhein (195)<smiles>O=C(O)C1=C[C@H](O)[C@H](O)[C@H](O)C1</smiles>

Shikimic Acid (197)

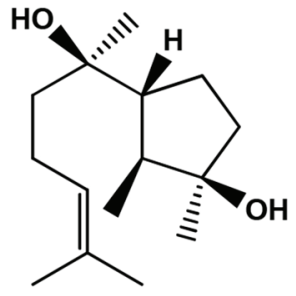

Cyclonerodiol (200)<smiles>COC(=O)c1oc(=O)c2c(O)c3c(=O)cc(C)oc3cc2c1O</smiles>

Lateropyrone (192)<smiles></smiles>

Sanguinarine (196)<smiles>CC1=C[C@H]2O[C@H](C/C=C(\C)C(=O)O)[C@@H](C)[C@H]2CC1</smiles>

Trichoderic Acid (198)

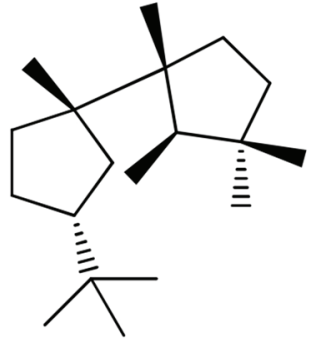

Cyclonerodiol oxide (201)<smiles>C/C=C/C=C/C(=O)C1CC(C)C(O)C(C)C1O</smiles>

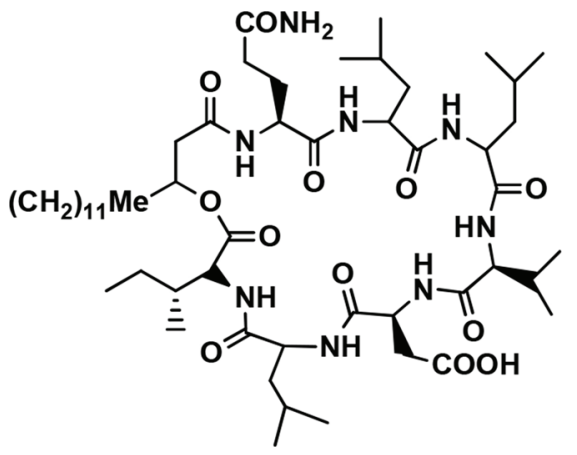

Halobacillin (204) 
Rhein (195) (Figure 14) was isolated from an endophyte Fusarium solani of Rheum palmatum collected at Ruoergai County, Sichuan Province, China. Rhein (195) naturally occurs in anthraquinone (1,3,8-trihydroxy-6-Me anthraquinone), that is found in Rheum palmatum L. and related plants such as rhubarb (You et al., 2013). It has good antibacterial activity with MICs in the range of $0.6-4 \mu \mathrm{g} / \mathrm{mL}$ against $S$. aureus, $S$. aureus nor $A$, B. megaterium 11561, Pseudomonas syringae and Sinorhizobium meliloti (Tegos et al., 2002).

Sanguinarine (196) (Figure 14), a benzophenanthridine alkaloid was obtained from the endophyte Fusarium proliferatum (strain BLH51) present on the leaves of Macleaya cordata of the Dabie Mountain, China. It has antibacterial, anthelmintic, and anti-inflammatory activities (Wang et al., 2014). It has antibacterial activities against the range of bacteria with MICs of 3.12$6.25 \mu \mathrm{g} / \mathrm{mL}$ against 15 clinical isolates of $S$. aureus while the MICs against of the two reference strains are $3.12 \mu \mathrm{g} / \mathrm{mL}$ for ATCC 25923 and $1.56 \mu \mathrm{g} / \mathrm{mL}$ for ATCC 33591.

The clinical isolates strains showed MIC values ranging from 31.25 to $250 \mu \mathrm{g} / \mathrm{mL}$ for ampicillin and $125-1000 \mu \mathrm{g} / \mathrm{mL}$ for ciprofloxacin. The treatment of the cells with sanguinarine induced the release of membrane-bound cell wall autolytic enzymes, which eventually resulted in lysis of the cell. Transmission electron microscopy (TEM) of MRSA treated with Sanguinarine show alterations in septa formation. The predisposition of lysis and altered morphology seen by TEM indicates that sanguinarine acts on the cytoplasmic membrane (ObiangObounou et al., 2011). The compound also has activity against plaque bacteria with MICs of $1-32 \mu \mathrm{g} / \mathrm{mL}$ for most species tested. The Electron microscopic studies of bacteria exposed to sanguinarine show that they aggregate and become morphologically irregular (Godowski, 1989).

Shikimic acid (197) (Figure 14), was obtained from the endophyte Trichoderma ovalisporum strain PRE-5 of the root of the herbal Panax notoginseng. The compound (197) is activity against S. aureus, Bacillus cereus, M. luteus and E. coli (Dang et al., 2010).

Trichoderic acid (198), 2 $\beta$-Hydroxytrichoacorenol (199), Cyclonerodiol (200), Cyclonerodiol oxide (201), and Sorbicillin (202) (Figure 14), were isolated from a Trichoderma sp. PR-35, an endophyte of Paeonia delavayi. These compounds are active against $E$. coli and $S$. albus with minimal inhibitory amount (MIA) values in the range of 25-150 mg/disk. Compounds (198), (200) and (201) are active against Shigella sonnei with MIA values in the range of 100-150 $\mu \mathrm{g} /$ disk (Wu et al., 2011).

Cyclopeptides PF1022F (203) and Halobacillin (204) (Figure 14), were isolated from the endophyte Trichoderma asperellum from traditional Chinese medicinal plant Panax notoginseng. Compounds (203) and (204) are active against E. faecium (CGMCC 1.2025) with $\mathrm{IC}_{50}$ values of 7.30 and $5.24 \mu \mathrm{M}$ and against $S$. aureus COL (CGMCC 1.2465) with $\mathrm{IC}_{50}$ values of 19.02 and $14.00 \mu \mathrm{M}$, respectively (Ding et al., 2012).

Tetrahydrobostrycin (205), 4-Deoxytetrahydrobostrycin (206), 3,6,8-Trihydroxy-1- methylxanthone (207), Griseophenone C (208) and 2,3-Didehydro-19 $\alpha$-hydroxy14-epicochlioquinone B (209) (Figure 15), were isolated from the endophyte Nigrospora sp. MA75, of the mangrove plant Pongamia pinnata collected from Guangxi Zhuang Autonomous
Region of China. Compound (209) has excellent activity against all the tested bacteria (MRSA, E. coli, P. aeruginosa, P. fluorescens and $S$. epidermidis) with MIC values of $8,4,4,0.5$, and $0.5 \mu \mathrm{g} / \mathrm{mL}$, respectively. The activity toward E. coli, P. fluorescens and S. epidermidis was stronger than that of the positive control (Ampicillin, with MICs values of 8,4 , and $4 \mu \mathrm{g} / \mathrm{mL}$, respectively). Compound (208) strongly inhibits MRSA, E. coli, P. aeruginosa, and $P$. fluorescens at MIC values of $0.5,2,0.5$, and $0.5 \mu \mathrm{g} / \mathrm{mL}$, respectively. Compound (205) has significant activity toward MRSA and E. coli (MIC 2 and $0.5 \mu \mathrm{g} / \mathrm{mL}$, respectively), while its analog compound (206), is only activity against E. coli (MIC $4 \mu \mathrm{g} / \mathrm{mL})$. This indicates that the $\mathrm{OH}$ group at $\mathrm{C}(4)$ could be important for the activity against MRSA. Compound (207) is active only against $S$. epidermidis (MIC $0.5 \mu \mathrm{g} / \mathrm{mL}$ ) (Shang et al., 2012).

4-Deoxybostrycin (210) and its derivative Nigrosporin (211) (Figure 15), were isolated from the mangrove endophyte Nigrospora sp. of the South China Sea. These compounds are active against $M$. tuberculosis and clinical multidrug-resistant (MDR) M. tuberculosis strains with MIC values of $<5->$ $60 \mu \mathrm{g} / \mathrm{mL}$ (Wang et al., 2013b).

Periconicins A (212) and B (213) (Figure 15), were isolated from an endophyte Periconia sp., from the branches of Taxus cuspidata. Periconicin A (212) has significant activity against B. subtilis, S. aureus, K. pneumoniae, and Salmonella typhimurium with MICs in the range of 3.12-12.5 $\mu \mathrm{g} / \mathrm{mL}$. Periconicin B (213) has modest antibacterial activity against the same strains with MICs in the range 25-50 $\mu \mathrm{g} / \mathrm{mL}$ (Kim et al., 2004).

Piperine (214) (Figure 15), which was originally isolated from Piper longum, was also detected from the endophyte Periconia sp. of the same plant. Piperine has strong activity against M. tuberculosis and M. smegmetis with MICs of 1.74 and $2.62 \mu \mathrm{g} / \mathrm{mL}$ respectively (Verma et al., 2011).

Modiolide A, 5, 8-dihydroxy-10-methyl-5, 8, 9, 10tetrahydro-2H-Oxecin-2-one (215) and 4-Chromanone, 6-hydroxy-2-methyl- (5CI) (216) (Figure 15) were isolated from the endophyte Periconia siamensis (strain CMUGE015) of the leaves of the grass, Thysanoleana latifolia (Poaceae). Compound (215) is active against Bacillus cereus, Listeria monocytogenes, MRSA, P. aeroginosa and E. coli with MIC of 3.12, 6.25, 25.00, 12.50, and $50.00 \mu \mathrm{g} / \mathrm{mL}$ respectively. Compound (216) is active against $B$. cereus, Listeria monocytogenes, MRSA, $P$. aeruginosa and E. coli with MICs of 6.25, 12.50, $50.0025 .00,12.50$, and $100.00 \mu \mathrm{g} / \mathrm{mL}$ respectively (Bhilabutra et al., 2007).

Xanalteric acids I (217) and II (218) (Figure 15) and Altenusin (219) (Figure 16), were obtained from Alternaria sp., of the mangrove plant Sonneratia alba. These (217-218) has weak antibacterial activities against MRSA with MICs of 125 and $250 \mu \mathrm{g} / \mathrm{mL}$. Altenusin (219) exhibited broad antimicrobial activity against several resistant pathogens (MRSA, S. pneumonia, E. faecium, E. cloacae and A. faecalis) with MIC values of $31.25-125 \mu \mathrm{g} / \mathrm{mL}$ (Kjer et al., 2009).

1-(2, 6-dihydroxyphenyl) butan-1-one (220) (Figure 16), was isolated from the endophyte Nodulisporium sp. of Juniperus cedrus from Gomera Island. Compound (220) is active against B. megaterium at $0.25 \mathrm{mg} /$ filter disc with $15 \mathrm{~mm}$ zone of inhibition (Dai et al., 2006). 
<smiles>[R]C1[C@H](O)[C@@H](O)C[C@H]2[C@@H](O)c3c(O)c(OC)cc(O)c3C(=O)[C@H]12</smiles>

$\mathrm{R}=\mathrm{OH}$ Tetrahydrobostrycin (205) $\mathrm{R}=\mathrm{H}$ Deoxytetrahydrobostrycin (206)

$\mathrm{HO}$<smiles>CC[C@H](C)C(=O)[C@H](C)C1=CC(=O)C2=C(O[C@]3(C)CC[C@@H]4O[C@H](C(C)(C)O)C[C@H](O)[C@]4(C)C3C2=O)C1=O</smiles>

2,3-didehydro-19ahydroxy14-epicochlioquinone B (209)<smiles>Cc1cc(O)cc2oc3cc(O)cc(O)c3c(=O)c12</smiles>

3,6,8-trihydroxy-1 -methylxanthone (207)<smiles>COc1cc(O)c2c(c1O)C(=O)C1=C(CC(O)C(C)(O)C1)C2=O</smiles>

4-deoxybostrycin (210)<smiles>COc1cc(O)c(C(=O)c2ccc(O)cc2OC)c(O)c1</smiles>

Griseophenone C (208)<smiles>COC1=CC(=O)c2c(cc3c(c2O)C[C@@H](O)[C@@H](O)C3)C1=O</smiles>

Nigrosporin (211)

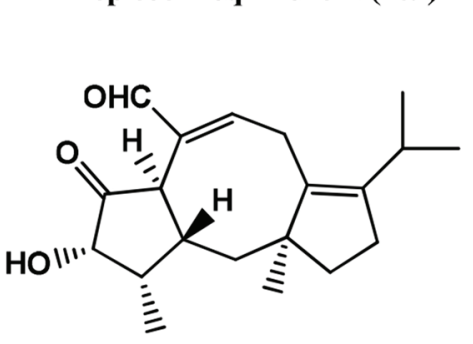

Periconicin A (212)<smiles>CC(CO)C1=C2C/C=C(/C=O)[C@@H]3C(=O)[C@H](O)[C@@H](C)[C@H]3C[C@@]2(C)CC1</smiles>

Periconicin B (213)<smiles>O=C(/C=C/C=C/c1ccc2c(c1)OCO2)N1CCCCC1</smiles>

Piperine (214)<smiles>CO[C@H](C)C[C@@H](O)/C=C/[C@H](O)/C=C\C(=O)O</smiles>

Modiolide A (215)<smiles>CC1CC(=O)C2C=C(O)C=CC2O1</smiles>

4-Chromanone,6-hydroxy-2methyl-(5CI) (216)<smiles>O=C(Cl)C1Oc2c(O)ccc3c1c1c4c(O)ccc1c(c23)C(O)C(C(=O)O)O4</smiles>

Xanalteric acid I (217)<smiles>O=C1C=Cc2c3c4c(c(O)ccc4c4ccc(O)c1c24)OC(C(=O)O)C3</smiles>

Xanalteric acid II (218)

FIGURE 15 | Structures of antibacterial metabolites isolated from Hyphomycetes (205-218).

Nodulisporins D-F (221-223), Benzene- 1, 2, 3-triol (224) (Figure 16), were isolated from an endophyte Nodulisporium sp. of Erica arborea. Compounds (221-224) showed activity against B. megaterium (Dai et al., 2009b).

Pyrrocidine (113) (Figure 9), was isolated from Acremonium zeae an endophyte of maize. Compound (113) has potent activity against Clavibacter michiganense subsp. Nebraskense a causal agent of Goss's bacterial wilt of maize (MICs $1-2 \mu \mathrm{g} / \mathrm{mL}$ ), as well as Bacillus mojavensis (MICs 1-2 $\mu \mathrm{g} / \mathrm{mL}$ ) and P. fluorescens (MICs 1-2 $\mu \mathrm{g} / \mathrm{mL}$ ) (Wicklow and Poling, 2009).

Rhizoctonic acid (139), Monomethylsulochrin (138)

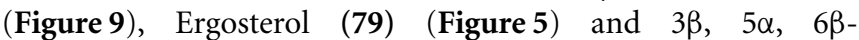
trihydroxyergosta-7, 22-diene (225) (Figure 16), were isolated from a Rhizoctonia sp. (Cy064), the endophyte in the leaves of Cynodon dactylon. Compounds $(139,138,79$, and 225) are active against five clinical and one reference strain of $H$. pylori (ATCC 
<smiles>COc1cc(C)c(C(=O)O)c(-c2cc(O)c(O)cc2C)c1</smiles>

Altenusin (219)<smiles>CCCC(=O)c1c(O)ccc([C@H]2C[C@H](C)Oc3cccc(O)c32)c1O</smiles>

Nodulisporin F (223)<smiles>CCCC(=O)c1c(O)cccc1O</smiles>

1-(2,6-dihydroxyphenyl) butan-1-one (220)<smiles>Oc1cccc(O)c1O</smiles>

Benzene-1,2,3-triol (224)<smiles>COc1cccc2c([C@@H]3C[C@H](C)Oc4cccc(O)c43)ccc(O)c12</smiles>

Nodulisporin D (221)<smiles>COc1cccc2ccc([C@@H]3C[C@H](C)Oc4cccc(O)c43)c(O)c12</smiles>

Nodulisporin E (222)<smiles>CC(C)[C@@H](C)/C=C/[C@@H](C)[C@H]1CCC2C3=C[C@H](O)[C@@]4(O)C[C@@H](O)CC[C@]4(C)C3CC[C@]21C</smiles>

$3 \beta, 5 \alpha, 6 \alpha$-trihydroxyergosta-7,22-diene (225)

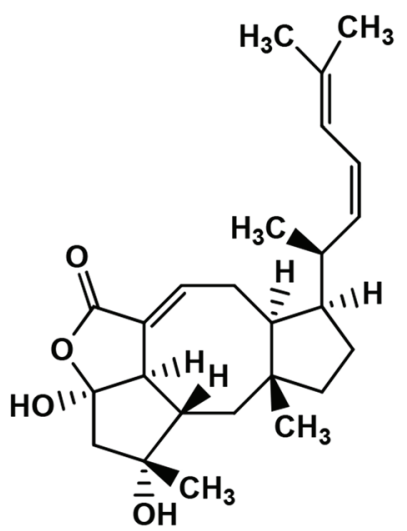

Ophiobolin P (226)<smiles></smiles>

Ophiobolin T (227)<smiles>COc1cc(O)cc2c1C(=O)CC(C)CCCC2</smiles>

(3S)-lasiodiplodin (228)<smiles>[R3]c1ccc(O)c2c1C([R2])([R2])[C@@H](C)OC2=O</smiles>

$\mathbf{R}_{\mathbf{1}}=\mathbf{R}_{\mathbf{2}}=\mathbf{R}_{\mathbf{3}}=\mathbf{H}$, (R)-(-)-mellein (229)

$\mathbf{R}_{\mathbf{1}}=\mathbf{R}_{\mathbf{3}}=\mathbf{H}, \mathbf{R}_{\mathbf{2}}=\mathrm{OH}$, cis-(3R,4R)-(-)-4-hydroxymellein (230)

$\mathbf{R}_{1}=\mathrm{OH}, \mathbf{R}_{\mathbf{2}}=\mathbf{R}_{3}=\mathrm{H}$, trans-(3R,4S)-(-)-4-hydroxymellein (231)

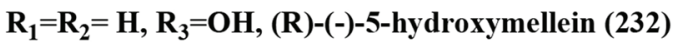

FIGURE 16 | Structures of antibacterial metabolites isolated from Hyphomycetes (219-232). 
43504) with MICs in the range $10.0-30.0 \mu \mathrm{g} / \mathrm{mL}$ (Ma et al., 2004).

Ophiobolins P (226) and T (227) (Figure 16), were isolated from the endolichenic fungus Ulocladium sp. Ophiobolins $\mathrm{P}$ has moderate antibacterial activity against $B$. subtilis and MRSA with MICs of 62.5 and $31.3 \mu \mathrm{g} / \mathrm{mL}$ respectively. Ophiobolin T (227) has moderate activity against B. subtilis and MRSA and Bacille Calmette-Guerin strain with MICs of 31.315 .6 and $31.3 \mu \mathrm{g} / \mathrm{mL}$ respectively (Wang et al., 2013a).

The antibacterial naphthaquinone Javanicin (181) (Figure 13) was isolated from an endophyte Chloridium sp. of Azadirachta indica. This compound is very active against $P$. fluorescens and P. aeruginosa with MIC of $2 \mu \mathrm{g} / \mathrm{mL}$ (Khrawar et al., 2009).

(3S)-Lasiodiplodin (228), (R)-(-)-Mellein (229), Cis-(3R, 4R)-(-)-4-Hydroxymellein (230), trans-(3R, 4S)-(-)-4Hydroxymellein (231), (R)-(-)-5-Hydroxymellein (232) (Figure 16) were isolated from the endophyte Botryosphaeria rhodina PSU-M35 and PSU-M114. Compound (228) is very active against $S$. aureus and MRSA with MIC values of 64 and $128 \mu \mathrm{g} / \mathrm{mL}$ respectively. Compounds (229-232) have much weaker activities than compound (228) with MIC values $>128 \mu \mathrm{g} / \mathrm{mL}$ (Rukachaisirikul et al., 2009).

Fusidilactones D (233) and E (234) (Figure 17) were isolated from the endophyte, a Fusidium sp. from the leaves of Mentha arvensis growing in a meadow near Hahausen, Lower Saxony, Germany. Both compounds are weakly active against E. coli and B. megaterium (Qin et al., 2009).

Palmariol B (235), 4-Hydroxymellein (236), Alternariol 9methyl ether (237) and Botrallin (238) (Figure 17) were isolated from an endophyte, Hyalodendriella sp. Ponipodef 12, of the hybrid "Neva" of Populus deltoides Marsh $\times$ P. nigra L. MIC 50 values of the compounds on Agrobacterium tumefaciens ranged from 18.22 to $87.52 \mu \mathrm{g} / \mathrm{mL}$. Against B. subtilis, P. lachrymans, R. solanacearum and $X$. vesicatoria, $\mathrm{MICs}_{50}$ were from 19.22 to $98.47,16.18$ to $92.21,16.24$ to 85.46 and 17.81 to $86.32 \mu \mathrm{g} / \mathrm{mL}$ respectively (Meng et al., 2012).

Alterporriol N (239), Alterporriol D (240), and Alterporriol E (241) (Figure 17), were isolated from Stemphylium globuliferuman an endophyte of Mentha pulegium collected from Morocco. Alterporriol N (239) is active against MRSA and E. faecalis with MICs of 62.5 and $15.63 \mu \mathrm{g} / \mathrm{mL}$. Alterporriol D (240) is active against MRSA and Streptomyces pneumonia with an MIC of $31.25 \mu \mathrm{g} / \mathrm{mL}$. Alterporriol E (241) is active against S. pneumonia, E. faecalis and Enterobacter cloacae with MICs of $31.25 \mu \mathrm{g} / \mathrm{mL}$ each (Debbab et al., 2009).

\section{COMPOUNDS PRODUCED FROM UNIDENTIFIED FUNGI}

Nonsporulating fungi form a major group of such endophytes. Khafrefungin, Arundifungin are antifungals reported from such fungi (Deshmukh and Verekar, 2012). Bostrycin (242) (Figure 18) isolated from the mangrove endophyte, no. 1403, of the South China Sea (Xu et al., 2010a), shows antibacterial activity against B. subtilis (Charudattan and Rao, 1982).

Guanacastepene A (243) (Figure 18), a novel diterpenoid produced the fungus CR115 isolated from the branch of Daphnopsis americana growing in Guanacaste, Costa Rica, may prove to belong to potentially new class of antibacterial agents with activities against MRSA and VRE (Singh et al., 2000). Guanacastepene I (244) (Figure 18), was isolated from the same fungus is active against $S$. aureus (Brady et al., 2001).

Anhydrofusarubin (245) (Figure 18), was isolated from the endophyte no. B77 of a mangrove tree on the South China Sea coast. Compound (245) is active against Staphylococcus aureus (ATCC27154) with a MIC of $12.5 \mu \mathrm{g} / \mathrm{mL}$ (Shao et al., 2008b).

3-O-Methylfusarubin (182) (Figure 13), Fusarubin (246) (Figure 18), were isolated from the endophyte B77 present in the seeds of the mangrove plant Kandelia candel in Zhanjiang. Compounds (182) and (246) were active against $S$. aureus ATCC 27154 with MIC values of 50.0 and $12.5 \mu \mathrm{g} / \mathrm{mL}$, respectively (Shao et al., 2008a).

Compound (247), 9 $\alpha$-Hydroxyhalorosellinia A (248) and Desoxybostrycin (249) (Figure 18), were isolated from the endophyte PSU-N24 present in the plant Garcinia nigrolineata collected from the Ton Nga Chang wildlife sanctuary, Songkhla province, southern Thailand. Compound (248) was active against M. tuberculosis with the MIC value of $12.50 \mu \mathrm{g} / \mathrm{mL}$ whilst compounds (247) and (249) had MIC values of 25 and $50 \mu \mathrm{g} / \mathrm{mL}$, respectively (Sommart et al., 2008).

Indolyl-3-carboxylic acid (250) (Figure 18), isolated from the endophyte S20 of Cephalotaxus hainanensis Li. showed inhibition of $S$. aureus and MRSA with diameters of inhibition zones of which were 12 and $8 \mathrm{~mm}$, respectively when $50 \mu \mathrm{l}$ of the compound $(10 \mathrm{mg} / \mathrm{mL})$ impregnated on sterile filter paper discs (6-mm diameter) (Dai et al., 2009a). The structure of a new 5acyl-2-methylpyrrole (251) (Figure 18) from the same endophyte S20 of Cephalotaxus hainanensis, was shown to be 1-(5-methyl1H-pyrrol-2-yl)-2-((2S*, 3R*)-3-((E)-prop-1-enyl) oxiran-2-yl) ethanone. Compound (251) is active against $S$. aureus and MRSA. The diameters of inhibition are $12.0 \mathrm{~mm}$ and $10.0 \mathrm{~mm}$ respectively when $50 \mu \mathrm{L}(10 \mathrm{mg} / \mathrm{mL})$ of the compound was impregnated on sterile filter paper discs (6-mm diameter) (Dai et al., 2009c).

Spirobisnaphthalenes, namely Diepoxin $\kappa$ (252), Diepoxin $\eta$ (253), and Diepoxin $\zeta$ (254) (Figure 18), were isolated from the endophyte Dzf12 of the medicinal plant Dioscorea zingiberensis. Among these, compound (252) has antibacterial activity, against E. coli, A. tumefaciens, X. vesicatoria, P. lachrymans and B. subtilis with MICs from 50 to $100 \mu \mathrm{g} / \mathrm{mL}$. A mixture of diepoxin $\eta$ (253), and diepoxin $\zeta$ (254) showed antibacterial activity against the same set of bacteria with a MICs range of 5.0-12.5 $\mu \mathrm{g} / \mathrm{mL}$ (Cai et al., 2009).

4-Hydroxyphthalide (255), 5-methoxy-7-hydroxyphthalide (256), (3R, 4R)-cis-4 hydroxymellein (257) (Figure 19), were obtained from an unidentified Ascomycete from Meliotus dentatus of the coastal area of the Baltic Sea, Ahrenshoop, Germany. Compounds (255) and (256) were active against E. coli whereas (256) and (257) were active against B. megaterium (Hussain et al., 2009b).

Pestalotheols E-H (258-261) and Anofinic acid (262) (Figure 19), were obtained from an unidentified ascomycete of Arbutus unedo. Compounds (258-262) have antibacterial activity against E. coli and B. megaterium (Qin et al., 2011).

Guignardone I (263) and Guignardone B (264) (Figure 19), were isolated from an endophyte fungus A1 of the mangrove plant Scyphiphora hydrophyllacea. Guignardone I (263) shows zones 
<smiles>CC[C@](C)(O)C[C@H](C)/C=C\[C@H]1OC2=C(C(=O)O[C@@H]2C)[C@@H](O)[C@@H]1O</smiles>

Fusidilactone D (233)<smiles>CC1OC(=O)c2c(O)cccc2C1O</smiles>

4-Hydroxymellein (236)<smiles></smiles>

Alterporriol N (239)<smiles>CC[C@H](C)C[C@@H](C)/C=C\[C@H]1O[C@H](O)[C@H](O)[C@H](O)[C@H]1C</smiles>

Fusidilactone E (234)<smiles>COc1cc(O)c2c(=O)oc3cc(O)c(Cl)c(C)c3c2c1</smiles>

Palmariol B (235)<smiles>COc1cc(O)c2c(=O)oc3cc(O)cc(C)c3c2c1</smiles>

Alternariol 9-methyl ether (237)<smiles>COC1=CC2OC(=O)c3c(O)cc(OC)cc3C2=C(O)C1=O</smiles>

Botrallin (238)

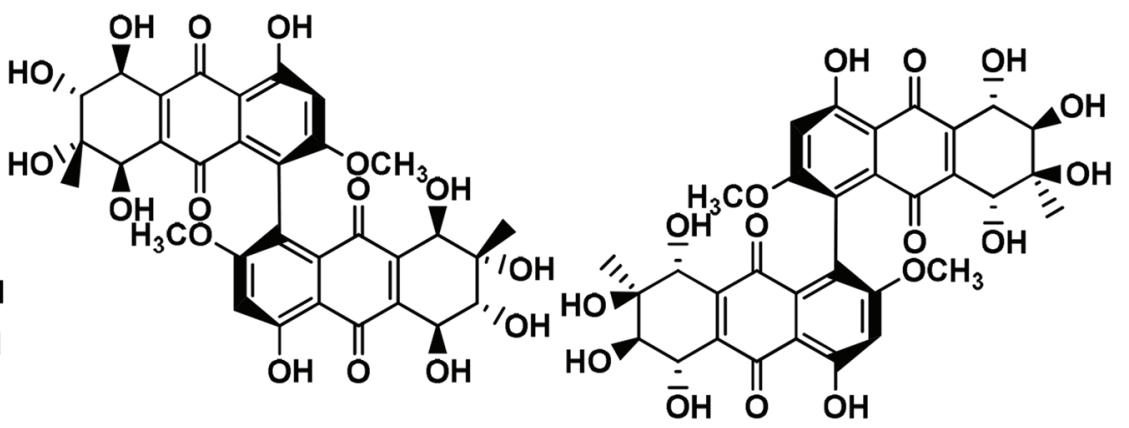

Alterporriol D (240)

Alterporriol E (241)

FIGURE 17 | Structures of antibacterial metabolites isolated from Hyphomycetes (233-241).

inhibition of 9.0 and $11.0 \mathrm{~mm}$ in diameter, using $6 \mathrm{~mm}$ filter paper discs toward MRSA and S. aureus at $65 \mu \mathrm{M}$, respectively. Guignardone B (264) shows zones of $8.0 \mathrm{~mm}$ against MRSA at $65 \mu \mathrm{M}$. Kanamycin sulfate, used as positive control $(10 \mu \mathrm{L}$ of $0.08 \mathrm{mg} / \mathrm{mL}$ ) showed an inhibition zone of $30 \mathrm{~mm}$ (Mei et al., 2012).

Mirandamycin (265) (Figure 19) was obtained from isolate 1223-D, an unclassified fungus of twig of Neomirandea angularis of family Asteraceae. It is active against E.coli 25922, $P$. aeruginosa 27853 , K. pneumoniae carbapenemase positive BAA1705, MRSA BAA-976 and $V$. cholerae PW357 with MICs of $80,80,>80,10$, and $40 \mu \mathrm{g} / \mathrm{mL}$ respectively (Ymele-Leki et al., 2012).

\section{Volatile organic compounds from endophytic fungi}

Strobel et al. (2001) reported at least 28 volatile organic compounds (VOC) from the xylariaceaous endophyte Muscodor albus (isolate 620), of Cinnamomum zeylanicum from Lancetilla Botanical Garden near La Ceiba, Honduras. These VOC's are mixtures of gasses of five class's viz. alcohols, acids, esters, ketones and lipids. The most effective were the esters, of which, 1butanol, 3-methyl-acetate has the highest activity. The VOC's inhibited and killed certain bacteria, within a period of 1-3 days. Most test organisms were completely inhibited, and in fact killed. These includes Escherichia coli, Staphylococcus aureus, Micrococcus luteus and Bacillus subtilis along with some fungal species. 
<smiles>COC1=CC(=O)c2c(O)c3c(c(O)c2C1=O)C[C@@](C)(O)[C@H](O)C3</smiles>

Bostrycin (242)<smiles>CC(=O)O[C@H]1C(=O)C2=CC3=C(C=O)[C@@H](O)CCC3(C)CC[C@]2(C)[C@H]1C(C)C</smiles>

Guanacastepene A (243)<smiles>CO[C@H]1C(=O)C2=C3O[C@H](O)C4=C3[C@@](C)(CC[C@@H]4O)[C@@]2(C)CC[C@]1(C)C(C)C</smiles>

Guanacastepene I (244)<smiles>COC1=CC(=O)c2c(O)c3c(c(O)c2C1=O)C=C(C)OC3</smiles>

Anhydrofusarubin (245)<smiles>O=C(O)c1c[nH]c2ccccc12</smiles>

Indolyl-3-carboxylic acid (250)<smiles>CC1=CCC2C(=O)CC[C@@H]3O[C@@H]3C[C@H](C)N12</smiles>

5-acyl-2-methylpyrrole (251)<smiles>COC1=CC(=O)c2c(O)c3c(c(O)c2C1=O)CC(C)(O)OC3</smiles>

Fusarubin (246)

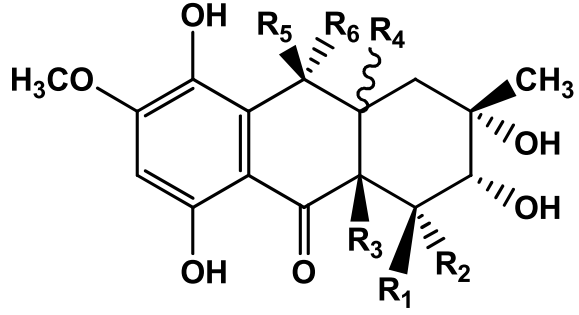

$\mathbf{R}_{\mathbf{1}}=\mathbf{R}_{\mathbf{2}}=\mathbf{R}_{\mathbf{3}}=\mathbf{R}_{\mathbf{5}}=\mathbf{H}, \mathbf{R}_{\mathbf{4}}=\boldsymbol{\beta}-\mathbf{H}, \mathbf{R}_{\mathbf{6}}=\mathbf{O H}$, Compound 2 (247)

$\mathbf{R}_{\mathbf{1}}=\mathbf{O H}, \mathbf{R}_{\mathbf{2}}=\mathbf{R}_{\mathbf{3}}=\mathbf{R}_{\mathbf{5}}=\mathrm{H}, \mathbf{R}_{\mathbf{4}}=\alpha-\mathrm{H}, \mathbf{R}_{\mathbf{6}}=\mathbf{O H}$, 9 $\alpha$-hydroxyhalorosellinia (248)

$\mathbf{R}_{\mathbf{1}}=\mathbf{R}_{\mathbf{2}}=\mathbf{H}, \mathbf{R}_{\mathbf{3}}=\mathbf{R}_{\mathbf{4}}=$ double bond,

$R_{5}+R_{6}=$ O Desoxybostrycin (249)<smiles></smiles>

Diepoxin $\kappa(252)$<smiles>O=C1CC[C@@H](O)C23OC45[C@@H](O)C6OC6C4(Oc4cccc6cccc(c46)O1)C25O3</smiles>

Diepoxin $\eta(253)$<smiles>O=C1C=C[C@@H](O)[C@@]23C[C@@H](O)C4OC4C4(Oc5cccc6cccc(c56)O4)C12O3</smiles>

Diepoxin $\xi$ (254)<smiles>[R2]c1cc([R2])c2c(c1[R3])COC2=O</smiles>

$\mathbf{R}_{\mathbf{1}}=\mathbf{R}_{\mathbf{2}}=\mathbf{H}, \mathbf{R}_{\mathbf{3}}=\mathbf{O H}$,

4-hydroxyphthalide (255) $\mathbf{R}_{1}=\mathrm{OH}, \mathrm{R}_{2}=\mathrm{OMe}, \mathrm{R}_{\mathbf{3}}=\mathrm{H}$, 5-methoxy-7-hydroxyphthalide (256)

FIGURE 18 | Structures of antibacterial metabolites isolated from Unidentified fungus (242-256). 
<smiles>C[C@@H]1OC(=O)c2c(O)cccc2[C@H]1O</smiles>

(3R,4R)-cis-4-hydroxymellein (257)<smiles>CC1(C)CC(=O)C2=C(C[C@H]3C[C@H](C(C)(C)O)O[C@H]3[C@H]2O)O1</smiles>

Pestalotheol E (258)<smiles>CC1(C)CC(=O)C2=C(C[C@H]3C[C@H](C(C)(C)O)O[C@H]3[C@H]2O)O1</smiles>

Pestalotheol F (259)<smiles>CC1(C)CC(=O)C2=C(O1)[C@@H]1O[C@@]13C[C@H](C(C)(C)O)O[C@H]2[C@H]3O</smiles>

Pestalotheol G (260)<smiles>CO[C@H]1C[C@@H](O)C2=C(C[C@@H]3[C@@H](C(C)(C)O)CC[C@]3(C)O2)C1=O</smiles>

Guignardone I (263)<smiles>CC(C)=CCC1=C[C@@H]2O[C@@H](C(C)(C)O)C[C@H]2CC1=O</smiles>

Pestalotheol H (261)<smiles>CC(C)(O)[C@H]1CC[C@]2(C)OC3=C(C[C@H]12)C(=O)[C@]1(O)CO[C@H]3C1</smiles>

Guignardone B (264)<smiles>CC1(C)C=Cc2cc(C(=O)O)ccc2O1</smiles>

Anofinic acid (262)<smiles>CCCc1c(O)ccc(O)c1CO</smiles>

Mirandamycin (265)

FIGURE 19 | Structures of antibacterial metabolites isolated from Unidentified fungus (257-265).

Strain of Muscodor namely Muscodor crispans of Ananas ananassoides (wild pineapple) growing in the Bolivian Amazon Basin produces VOC's; namely propanoic acid, 2-methyl-; 1butanol, 3-methyl-; 1-butanol, 3-methyl-, acetate; propanoic acid, 2-methyl-, 2-methylbutyl ester; and ethanol. The VOC's of this fungus are effective against Xanthomonas axonopodis pv. citri a citrus pathogens. The VOC's of M. crispans kill several human pathogens, including Yersinia pestis, Mycobacterium tuberculosis and Staphylococcus aureus. Muscodor crispans is only effective against the vegetative cells of Bacillus anthracis, but not against the spores. Artificial mixtures of the fungal VOC's were both inhibitory and lethal to a number of human and plant pathogens, including three drug-resistant strains of Mycobacterium tuberculosis (Mitchell et al., 2010). The mechanism of action of the VOC's of Muscodor spp. on target bacteria is unknown. A microarray study of the transcriptional response analysis of B. subtilis cells exposed to M. albus VOC's show that the expression of genes involved in DNA repair and replication increased, suggesting that VOC's induce some type of DNA damage in cells, possibly through the effect of one of the naphthalene derivatives (Mitchell et al., 2010).

\section{Outlook}

A definite, urgent and worldwide effort is needed to tackle the problems of the populations in third world and developing countries. MRSA, VRE, PRSP, ESCAPE organisms have spread through these countries over the years particularly due to immunocompromised populations. Mycobacterium tuberculosis is a major threat! and New and Novel drugs are a must!! Endophytic fungi may be an excellent source of such compounds. These organisms have a vast repertoire of diverse chemicals such as steroids, xanthones, phenols, isocoumarins, perylene derivatives, quinones, furandiones, terpenoids, depsipeptides and cytochalasins (Tan and Zou, 2001; Gunatilaka, 2006; Zhang et al., 2006; Guo et al., 2008).

A major challenge in Drug Discovery Program based on endophytic fungi lies in developing effective strategies to isolating bioactive strains. Strobel and Daisy (2003) suggested that areas of high biodiversity of endemic plant species may hold the greatest potential for endophytes with novel chemical entities. Tropical forests are some of the most bio diverse ecosystems and their leaves are "biodiversity hotspots" (Arnold and Lutzoni, 2007). The selection of plants is crucial. Those with medicinal properties 
should be given preference. Metabolites produced by fungi need to correlated with the plant genomics, thus allowing far better knowledge of biosynthetic pathways. This will also justify the production of metabolites rather than unproven hypotheses.

Identification of endophytic fungi using molecular analyses provides an opportunity to look for broad patterns in bioactivity not only at the genotype or strain level, but at higher taxonomic levels that may in turn assist in focusing on the association of metabolite with the plant.

The endophytic flora of the Indian subcontinent has been explored for their diversity but not enough for their bioactive metabolites. The published work is scanty (Puri et al., 2005; Deshmukh et al., 2009; Khrawar et al., 2009; Periyasamy et al., 2014). There is a need for groups from different scientific discipline (mycologist, chemist, toxicologist, and pharmacologist) to engage in this search process. Enormous natural wealth exists in the world's tropical forests, but disparity exists between developed countries with their financial resources and biodiversity rich countries with underdeveloped economy and limited funds. May be funding agencies need to look at such aspects.

The need of a more and larger collection of fungal endopytes is suggested. Bioactive metabolite metabolites from such collections could yield leads for pharmaceutical and agricultural application.

What emerges is the essential bonding of various discipline of biology and chemistry into cohesive target delivery vehicles.

\section{ACKNOWLEDGMENTS}

The authors are extremely grateful to Dr. B. N. Ganguli, Emeritus Scientist of the CSIR, India and Chair Professor of the Agharkar Research Institute, Pune, Fellow of the Biotechnology Research Institute of India for his very careful scrutiny and suggestions of this review paper.

\section{REFERENCES}

Ahmed, I., Hussain, H., Schulz, B., Draeger, S., Padula, D., Pescitelli, G., et al. (2011). Three new antimicrobial metabolites from the endophytic fungus Phomopsis sp. Eur. J. Org. Chem. 2011, 2867-2873, S2867/1-S2867/5. doi: 10.1002/ejoc.201100158

Aly, A. H., Debbab, A., and Proksch, P. (2011). Fungal endophytes: unique plant inhabitants with great promises. Appl. Microbiol. Biotechnol. 90, 1829-1845. doi: 10.1007/s00253-011-3270-y

Arnold, A. E., and Lutzoni, F. (2007). Diversity and host range of foliar fungal endophytes: are tropical leaves biodiversity hotspots? Ecology 88, 541-549. doi: $10.1890 / 05-1459$

Arunpanichlert, J., Rukachaisirikul, V., Sukpondma, Y., Phongpaichit, S., Tewtrakul, S., Rungjindamai, N., et al. (2010). Azaphilone and isocoumarin derivatives from the endophytic fungus Penicillium sclerotiorum PSU-A13. Chem. Pharm. Bull. 58, 1033-1036. doi: 10.1248/cpb. 58.1033

Augner, D., Krut, O., Slavov, N., Gerbino, D. C., Sahl, H. G., Benting, J., et al. (2013). On the antibiotic and antifungal activity of pestalone, pestalachloride A, and structurally related compounds. J. Nat. Prod. 76, 1519-1522. doi: $10.1021 / \mathrm{np} 400301 \mathrm{~d}$

Bacon, C. W., and White, J. F. (2000). Microbial Endophytes. New York, NY: Marcel Dekker Inc.

Bhilabutra, W., Techowisan, T., Peberdy, J. F., and Lumyong, S. (2007). Antimicrobial activity of bioactive compounds from Periconia siamensis CMUGE015. Res. J. Microbiol. 2, 749-755. doi: 10.3923/jm.2007. 749.755

Brady, S. F., Bondi, S. M., and Clardy, J. (2001). The guanacastepenes: a highly diverse family of secondary metabolites produced by an endophytic fungus. J. Am. Chem. Soc. 123, 9900-9901. doi: 10.1021/ja016176y
Brady, S. F., Wagenaar, M. M., Singh, M. P., Janso, J. E., and Clardy, J. (2000). The cytosporones, new octaketide antibiotics isolated from an endophytic fungus. Org. Lett. 2, 4043-4046. doi: 10.1021/ol006680s

Cai, X., Shan, T., Li, P., Huang, Y., Xu, L., Zhou, L., et al. (2009). Spirobisnaphthalenes from the endophytic fungus Dzf 12 of Dioscorea zingiberensis and their antimicrobial activities. Nat. Prod. Commun. 4, 1469-1472.

Casella, T. M., Eparvier, V., Mandavid, H., Bendelac, A., Odonne, G., Dayan, L., et al. (2013). Antimicrobial and cytotoxic secondary metabolites from tropical leaf endophytes: isolation of antibacterial agent pyrrocidine C from Lewiainfectoria SNB-GTC2402. Phytochemistry 96, 370-377. doi: 10.1016/j.phytochem.2013.10.004

Charudattan, R., and Rao, K. V. (1982). Bostrycin and 4-deoxybostrycin: two nonspecific phytotoxins produced by Alternaria eichhorniae. Appl. Environ. Microbiol. 43, 846-849.

Chen, H. J., Awakawa, T., Sun, J. Y., Wakimoto, T., and Abe, I. (2013). Epigenetic modifier-induced biosynthesis of novel fusaric acid derivatives in endophytic fungi from Datura stramonium L. Nat. Prod. Bioprospect. 3, 20-23. doi: 10.1007/s13659-013-0010-2

Chen, X., Sang, X., Li, S., Zhang, S., and Bai, L. (2010). Studies on a chlorogenic acid-producing endophytic fungi isolated from Eucommia ulmoides Oliver. J. Ind. Microbiol. Biotechnol. 37, 447-454. doi: 10.1007/s10295-010-0690-0

Chen, X., Shi, Q., Lin, G., Guo, S., and Yang, J. (2009). Spirobisnaphthalene analogs from the endophytic fungus Preussia sp. J. Nat. Prod. 72, 1712-1715. doi: $10.1021 / \mathrm{np} 900302 \mathrm{w}$

Cheng, M. J., Wu, M. D., Yanai, H., Su, Y. S., Chen, I. S., Yuan, G. F., et al. (2012). Secondary metabolites from the endophytic fungus Biscogniauxia formosana and their antimycobacterial activity. Phytochem. Lett. 5, 467-472. doi: 10.1016/j.phytol.2012.04.007

Choi, J. N., Kim, J., Ponnusamy, K., Lim, C., Kim, J. G., Muthaiya, M. J., et al. (2013). Identification of a new phomoxanthone antibiotic from Phomopsislongicolla and its antimicrobial correlation with other metabolites during fermentation. J. Antibiot. 66, 231-233. doi: 10.1038/ja.2012.105

Chomcheon, P., Wiyakrutta, S., Sriubolmas, N., Ngamrojanavanich, N., Isarangkul, D., and Kittakoop, P. (2005). 3-Nitropropionic acid (3-NPA), a potent antimycobacterial agent from endophytic fungi: is 3-NPA in some plants produced by endophytes? J. Nat. Prod. 68, 1103-1105. doi: 10.1021/np050036a

Cueto, M., Jensen, P. R., Kauffman, C., Fenical, W., Lobkovsky, E., and Clardy, J. (2001). Pestalone, a new antibiotic produced by a marine fungus in response to bacterial challenge. J. Nat. Prod. 64, 1444-1446. doi: 10.1021/np0102713

Dai, J., Krohn, K., Draeger, S., and Schulz, B. (2009b). New naphthalene-chroman coupling products from the endophytic fungus, Nodulisporium sp. from Erica arborea. Eur. J. Org. Chem. 2009, 1564-1569. doi: 10.1002/ejoc.200801106

Dai, J., Krohn, K., Floerke, U., Draeger, S., Schulz, B., Kiss-Szikszai, A., et al. (2006). Metabolites from the endophytic fungus Nodulisporium sp. from Juniperus cedre. Eur. J. Org. Chem. 15, 3498-3506. doi: 10.1002/ejoc.200600261

Dai, J., Krohn, K., Floerke, U., Gehle, D., Aust, H. J., Draeger, S., et al. (2005). Novel highly substituted biraryl ethers, phomosines D-G, isolated from the endophytic fungus Phomopsis sp. from Adenocarpus foliolosus. Eur. J. Org. Chem. 23, 5100-5105. doi: 10.1002/ejoc.200500471

Dai, W. J., Dai, H. F., Wu, J., Liu, J., and Mei, W. L. (2009a). A new 5-acyl-2methylpyrrole from the endophytic fungus S20 of Cephalotaxus hainanensis. Nat. Prod. Commun. 4, 1489-1490.

Dai, W. J., Wu, J., Han, Z., Mei, W. L., and Dai, H. F. (2009c). Metabolites from endophytic fungus S20 of Cephalotaxus hainanensis. J. Asian Nat. Prod. Res. 11, 703-708. doi: 10.1080/10286020902858846

Dang, L., Li, G., Yang, Z., Luo, S., Zheng, X., and Zhang, K. (2010). Chemical constituents from the endophytic fungus Trichoderma ovalisporum isolated from Panax notoginseng. Ann. Microbiol. 60, 317-320. doi: 10.1007/s13213-0100043-2

Debbab, A., Aly, A. H., Edrada-Ebel, R., Wray, V., Müller, W. E., Totzke, F, et al. (2009). Bioactive metabolites from the endophytic fungus Stemphylium globuliferum isolated from Mentha pulegium. J. Nat. Prod. 72, 626-631. doi: $10.1021 / \mathrm{np} 8004997$

Deshmukh, S. K., Mishra, P. D., Kulkarni-Almeida, V. S. A., Sahoo, M. R., Periyasamy, G., Goswami, H., et al. (2009). Anti-inflammatory and anti-cancer activity of Ergoflavin isolated from an endophytic fungus. Chem. Biodivers. 6, 784-789. doi: 10.1002/cbdv.200800103

Deshmukh, S. K., and Verekar, S. A. (2012). Fungal endophytes: a potential source of antifungal compounds. Front. Biosci. E4, 2045-2070. doi: 10.2741/E524 
Devi, P., Rodrigues, C., Naik, C. G., and D’Souza, L. (2012). Isolation and characterization of antibacterial compound from a mangrove-endophytic fungus, Penicillium chrysogenum MTCC 5108. Ind. J. Microbiol. 52, 617-623. doi: 10.1007/s12088-012-0277-8

Ding, G., Chen, A. J., Lan, J., Zhang, H., Chen, X., Liu, X., et al. (2012). Sesquiterpenes and Cyclopeptides from the Endophytic Fungus Trichoderma asperellum Samuels, Lieckf. and Nirenberg. Chem. Biodivers. 9, 1205-1212. doi: 10.1002/cbdv.201100185

Ding, G., Li, Y., Fu, S., Liu, S., Wei, J., and Che, Y. (2009). Ambuic acid and torreyanic acid derivatives from the endolichenic fungus Pestalotiopsis sp. J. Nat. Prod. 72, 182-186. doi: 10.1021/np800733y

Dreyfuss, M. M., and Chapela, I. H. (1994). "Potential of fungi in the discovery of novel, low-molecular weight pharmaceuticals," in The Discovery of Natural Products with Therapeutic Potential, ed V. P. Gullo (London: ButterworthHeinemann), 49-80.

Du, F. Y., Li, X. M., Li, C. S., Shang, Z., and Wang, B. G. (2012). Cristatumins A-D, new indole alkaloids from the marine-derived endophytic fungus Eurotium cristatum EN-220. Bioorg. Med. Chem. Lett. 22, 4650-4653. doi: 10.1016/j.bmcl.2012.05.088

Elsaesser, B., Krohn, K., Floerke, U., Root, N., Aust, H. J., Draeger, S., et al. (2005). X-ray structure determination, absolute configuration and biological activity of phomoxanthone A. Eur. J. Org. Chem. 2005, 4563-4570. doi: 10.1002/ejoc.200500265

Elsebai, M. F., Natesan, L., Kehraus, S., Mohamed, I. E., Schnakenburg, G., Sasse, F., et al. (2011). HLE-inhibitory alkaloids with a polyketide skeleton from the marine-derived fungus Coniothyrium cereal. J. Nat. Prod. 74, 2282-2285. doi: $10.1021 / \mathrm{np} 2004227$

Erbert, C., Lopes, A. A., Yokoya, N. S., Furtado, N. A. J. C., Conti, R., Pupo, M. T., et al. (2012). Antibacterial compound from the endophytic fungus Phomopsislongicolla isolated from the tropical red seaweed Bostrychia radicans. Bot. Mar. 55, 435-440. doi: 10.1515/bot-2011-0023

Freeman, E. M. (1904). The seed-fungus of lolium temulentum, L., the darnel. Philos. Trans. R. Soc. Lond. B 196, 1-27. doi: 10.1098/rstb.1904.0001

Gao, S., Li, X., Zhang, Y., Li, C., and Wang, B. (2011). Conidiogenones H and I, two new diterpenes of cyclopiane class from a marine-derived endophytic fungus Penicillium chrysogenum QEN-24S. Chem. Biodivers. 8, 1748-1753. doi: 10.1002/cbdv.201000378

Ge, H. M., Zhang, Q., Xu, S. H., Guo, Z., K., Song, Y. C., Tan, R. X., et al. (2011). Chaetoglocins A-D. Four new metabolites from the endophytic fungus Chaetomium globosum. Planta Med. 77, 277-280. doi: 10.1055/s-0030-1250292

Gillespie, S. H. (2002). Evolution of drug resistance in Mycobacterium tuberculosis: clinical and molecular perspective. Ant. Agents Chemother. 46, 267-274. doi: 10.1128/AAC.46.2.267-274.2002

Godowski, K. C. (1989). Antimicrobial action of Sanguinarine. J. Clin. Dent. 1, 96-101.

Gunatilaka, A. A. L. (2006). Natural products from plant-associated microorganisms: distribution, structural diversity, bioactivity and implications of their occurrence. J. Nat. Prod. 69, 509-526. doi: 10.1021/np058128n

Guo, B., Dai, J., Ng, S., Huang, Y., Leong, C., Ong, W., et al. (2000). Cytonic acids $\mathrm{A}$ and B: novel tridepside inhibitors of hCMV protease from the endophytic fungus Cytonaema species. J. Nat. Prod. 63, 602-604. doi: 10.1021/np990467r

Guo, B., Wang, Y., Sun, X., and Tang, K. (2008). Bioactive natural products from endophytes: a review. Appl. Biochem. Microbiol. 44, 136-142. doi: 10.1134/S0003683808020026

Harper, J. K., Ford, E. J., Strobel, G. A., Arif, A. M., Grant, D. M., Porco, J., et al. (2003). Pestacin: a 1,3-dihydro isobenzofuran from Pestalotiopsis microspora possessing antioxidant and antimycotic activities. Tetrahedron 59, 2471-2476. doi: 10.1016/S0040-4020(03)00255-2

Hawas, U. W., El-Beih, A. A., and El-Halawany, A. M. (2012). Bioactive anthraquinones from endophytic fungus Aspergillus versicolor isolated from red sea alga. Arch. Pharm. Res. 35, 1749-1756. doi: 10.1007/s12272-012-1006-x

Hawksworth, D. C., and Rossman, A. Y. (1997). Where are the undescribed fungi? Phytopathology 87, 888-891. doi: 10.1094/PHYTO.1997.87.9.888

Hemberger, Y., Xu, J., Wray, V., Proksch, P., Wu, J., and Bringmann, G. (2013). Pestalotiopens A and B: stereochemically challenging flexible sesquiterpenecyclopaldic acid hybrids from Pestalotiopsis sp. Chem. A Eur. J. 19, 15556-15564. doi: 10.1002/chem.201302204

Hoffman, A. M., Mayer, S. G., Strobel, G. A., Hess, W. M., Sovocool, G. W., Grange, A. H., et al. (2008). Purification, identification and activity of phomodione, a furandione from an endophytic Phoma species. Phytochemistry 69, 1049-1056. doi: 10.1016/j.phytochem.2007.10.031

Hu, Z. Y., Li, Y. Y., Lu, C. H., Lin, T., Hu, P., and Shen, Y. M. (2010). Seven novel linear polyketides from Xylaria sp. NCY2. Helv. Chim. Acta 93, 925-933. doi: 10.1002/hlca.200900323

Hussain, H., Ahmed, I., Schulz, B., Draeger, S., and Krohn, K. (2012a). Pyrenocines J-M: four new pyrenocines from the endophytic fungus, Phomopsis sp. Fitoterapia 83, 523-526. doi: 10.1016/j.fitote.2011.12.017

Hussain, H., Akhtar, N., Draeger, S., Schulz, B., Pescitelli, G., Salvadori, P., et al. (2009a). Biologically active secondary metabolites from fungi, 40. New bioactive 2,3-epoxycyclohexenes and isocoumarins from the endophytic fungus Phomopsis sp. from Laurus azorica. Eur. J. Org. Chem. 2009, 749-756. doi: 10.1002/ejoc.200801052

Hussain, H., Krohn, K., Ahmed, I., Draeger, S., Schulz, B., Di Pietro, S., et al. (2012b). Phomopsinones A-D: four new pyrenocines from endophytic fungus Phomopsis sp. Eur. J. Org. Chem. 2012, 1783-1789. doi: 10.1002/ejoc.201101788

Hussain, H., Krohn, K., Draeger, S., Meier, K., and Schulz, B. (2009b). Bioactive chemical constituents of a sterile endophytic fungus from Meliotus dentatus. Rec. Nat. Prod. 3, 114-117.

Hussain, H., Tchimene, M. K., Ahmed, I., Meier, K., Steinert, M., Draeger, S., et al. (2011). Antimicrobial chemical constituents from the endophytic fungus Phomopsis sp. from Notobasis syriaca. Nat. Prod. Commun. 6, 1905-1906.

Isaka, M., Jaturapat, A., Rukseree, K., Danwisetkanjana, K., Tanticharoen, M., and Thebtaranonth, Y. (2001). Phomoxanthones A and B, novel xanthone dimers from the endophytic fungus Phomopsis species. J. Nat. Prod. 64, 1015-1018. doi: $10.1021 /$ np010006h

Khrawar, R. N., Verma, V. C., Kumar, A., Gond, S. K., Harper, J. K., Hess, W. M., et al. (2009). Javanicin, an antibacterial naphthaquinone from an endophytic fungus of Neem, Chloridium sp. Curr. Microbiol. 58, 233-238. doi: 10.1007/s00284-008-9313-7

Kim, S., Shin, D. S., Lee, T., and Oh, K. B. (2004). Periconicins, two new fusicoccane diterpenes Produced by an endophytic fungus Periconia sp. with antibacterial activity. J. Nat. Prod. 67, 448-450. doi: 10.1021/np030384h

Kjer, J., Wray, V., Edrada-Ebel, R., Ebel, R., Pretsch, A., Lin, W., et al. (2009) Xanalteric acids I and II and related phenolic compounds from an endophytic Alternaria sp. isolated from the mangrove plant Sonneratia alba. J. Nat. Prod. 72, 2053-2057. doi: 10.1021/np900417g

Kornsakulkarn, J., Dolsophon, K., Boonyuen, N., Boonruangprapa, T., Rachtawee, P., Prabpai, S., et al. (2011). Dihydronaphthalenones from endophytic fungus Fusarium sp. BCC14842. Tetrahedron 67, 7540-7547. doi: 10.1016/j.tet.2011.07.078

Krohn, K., Farooq, U., Hussain, H., Ahmed, I., Rheinheimer, J., Draeger, S., et al. (2011). Phomosines H-J, novel highly substituted biaryl ethers, isolated from the endophytic fungus Phomopsis sp. from Ligustrum vulgare. Nat. Prod. Commun. 6, 1907-1912.

Krohn, K., Kouam, S. F., Cludius-Brandt, S., Draeger, S., and Schulz, B. (2008a). Bioactive nitronaphthalenes from an endophytic fungus, Coniothyrium sp., and their chemical synthesis. Eur. J. Org. Chem. 2008, 3615-3618. doi: 10.1002/ejoc. 200800255

Krohn, K., Kouam, S. F., Kuigoua, G. M., Hussain, H., Cludius-Brandt, S., Florke, U., et al. (2009). Xanthones and oxepino [2, 3-b]chromones from three endophytic fungi. Chemistry 15, 12121-12132. doi: 10.1002/chem.200900749

Krohn, K., Michel, A., Roemer, E., Floerke, U., Aust, H. J., Draeger, S., et al. (1995). Biologically active metabolites from fungi. 6. Phomosines A-C Three new biaryl ethers from Phomopsis sp. Nat. Prod. Lett. 6, 309-314. doi: 10.1080/10575639508043176

Krohn, K., Sohrab, M. H., van Ree, T., Draeger, S., Schulz, B., Antus, S., et al. (2008b). Biologically active secondary metabolites from fungi, 39. Dinemasones A, B and C: new bioactive metabolites from the endophytic fungus Dinemasporium strigosum. Eur. J. Org. Chem. 2008, 5638-5646. doi: 10.1002/ejoc. 200800688

Lacey, L. A., and Neven, L. G. (2006). The potential of the fungus, Muscodor albus, as a microbial control agent of potato tuber moth (Lepidoptera: Gelechiidae) in stored potatoes. J. Invertebr. Pathol. 91, 195-198. doi: 10.1016/j.jip.2006. 01.002

Lai, D., Broetz-Oesterhelt, H., Mueller, W. E. G., Wray, V., and Proksch, P. (2013). Bioactive polyketides and alkaloids from Penicillium citrinum, a fungal endophyte isolated from Ocimum tenuiflorum. Fitoterapia 91, 100-106. doi: 10.1016/j.fitote.2013.08.017 
Lee, J. C., Strobel, G. A., Lobkovsky, E., and Clardy, J. C. (1996). Torreyanic acid: a selectively cytotoxic quinone dimer from the endophytic fungus Pestalotiopsis microspora. J. Org. Chem. 61, 3232-3233. doi: 10.1021/jo960471x

Lee, J., Lobkovsky, E., Pliam, N. B., Strobel, G. A., and Clardy, J. (1995). Subglutinols A and B: immunosuppressive compounds from the endophytic fungus Fusarium subglutinans. J. Org. Chem. 60, 7076-7077. doi: 10.1021/jo00127a001

Li, J. Y., Harper, J. K., Grant, D. M., Tombe, B. O., Bashyal, B., Hess, W. M., et al. (2001). Ambuic acid, a highly functionalized cyclohexenone with antifungal activity from Pestalotiopsis spp. and Monochaetia sp. Phytochemistry 56, 463-468. doi: 10.1016/S0031-9422(00)00408-8

Li, J. Y., Strobel, G. A., Harper, J. K., Lobkovsky, E., and Clardy, J. (2000). Cryptocin, a potent tetramic acid antimycotic from the endophytic fungus Cryptosporiopsis cf. quercina. Org. Lett. 2, 767-770. doi: 10.1021/ol000008d

Li, M., Chang, M., Zhang, Q., He, R., and Ye, B. (2010). Endophytic fungus strain ZD6 isolated from the stem of Bruguiera gymnorrhiza and the antibacterial activity of its metabolites. Junwu Xuebao 29, 739-745.

Li, S., Wei, M., Chen, G., and Lin, Y. (2012). Two new dihydroisocoumarins from the endophytic fungus Aspergillus sp. collected from the South China Sea. Chem. Nat. Comp. 48, 371-373. doi: 10.1007/s10600-012-0254-9

Li, Y., Song, Y. C., Liu, J. Y., Ma, Y. M., and Tan, R. X. (2005). Anti-Helicobacter pylori substances from fungal cultures. World J. Microbiol. Biotechnol. 21, 553-558. doi: 10.1007/s11274-004-3273-2

Lim, C., Kim, J., Choi, J. N., Ponnusamy, K., Jeon, Y., Kim, S. U., et al. (2010). Identification, fermentation, and bioactivity against Xanthomonas oryzae of antimicrobial metabolites isolated from Phomopsis longicolla S1B4. J. Microbiol. Biotechnol. 20, 494-500. doi: 10.4014/jmb.0909.09026

Liu, X., Dong, M., Chen, X., Jiang, M., Lv, X., and Zhou, J. (2008). Antimicrobial activity of an endophytic Xylaria sp.YX-28 and identification of its antimicrobial compound 7-amino-4-methylcoumarin. Appl. Microbiol. Biotechnol. 78, 241-247. doi: 10.1007/s00253-007-1305-1

LoBue, P. (2009). Extensively drug-resistant tuberculosis. Curr. Opin. Infect. Dis. 22, 167-173. doi: 10.1097/QCO.0b013e3283229fab

Loesgen, S., Bruhn, T., Meindl, K., Dix, I., Schulz, B., Zeeck, A., et al. (2011). (+)Flavipucine, the missing member of the pyridione epoxide family of fungal antibiotics. Eur. J. Org. Chem. 2011, 5156-5162. doi: 10.1002/ejoc.201100284

Loesgen, S., Magull, J., Schulz, B., Draeger, S., and Zeeck, A. (2008). Isofusidienols: novel chromone-3-oxepines produced by the endophytic fungus Chalara sp. Eur. J. Org. Chem. 2008, 698-703. doi: 10.1002/ejoc.200700839

Lu, S., Draeger, S., Schulz, B., Krohn, K., Ahmed, I., Hussain, H., et al. (2011). Bioactive aromatic derivatives from endophytic fungus, Cytospora sp. Nat. Prod. Commun. 6, 661-666.

Lucas, E. M. F., Monteiro de Castro, M. C., and Takahashi, J. A. (2007).Antimicrobial properties of sclerotiorin, isochromophilone VI andpencolide, metabolites from a Brazilian cerrado isolate of Penicillium sclerotiorum van Beyma. Braz. J. Microbiol. 38, 785-789. doi: 10.1590/S1517-838220070 00400036

Luo, J., Liu, X., Li, E., Guo, L., and Che, Y. (2013). Arundinols A-C and Arundinones A and B from the plant endophytic fungus Microsphaeropsis arundinis. J. Nat. Prod. 76, 107-112. doi: 10.1021/np300806a

Ma, Y. M., Li, Y., Liu, J. Y., Song, Y. C., and Tan, R. X. (2004). Anti-Helicobacter pylori metabolites from Rhizoctonia sp. Cy064, an endophytic fungus in Cynodon dactylon. Fitoterapia 75, 451-456. doi: 10.1016/j.fitote.2004.03.007

Mei, W. L., Zheng, B., Zhao, Y. X., Zhong, H. M., Chen, X. L., Zeng, Y. B., et al. (2012). Meroterpenes from endophytic fungus Al of mangrove plant Scyphiphora hydrophyllacea. Mar. Drug. 10, 1993-2001. doi: 10.3390/md10091993

Melo, I. S., Santos, S. N., Rosa, L. H., Parma, M. M., Silva, L. J., Queiroz, S. C., et al. (2014). Isolation and biological activities of an endophytic Mortierella alpina strain from the Antarctic moss Schistidium antarctici. Extremophiles18, 15-23. doi: 10.1007/s00792-013-0588-7

Meng, X., Mao, Z., Lou, J., Xu, L., Zhong, L., Peng, Y., et al. (2012). Benzopyranones from the endophytic fungus Hyalodendriella sp. Ponipodef 12 and their bioactivities. Molecules 17, 11303-11314. doi: 10.3390/molecules171011303

Menichetti, F. (2005). Current and emerging serious Gram-positive infections. Clin. Microbiol. Infect. 11, 22-28. doi: 10.1111/j.1469-0691.2005.01138.x

Miao, F. P., Li, X. D., Liu, X. H., Cichewicz, R. H., and Ji, N. Y. (2012). Secondary metabolites from an algicolous Aspergillus versicolor strain. Mar. Drug. 10, 131-139. doi: 10.3390/md10010131
Mitchell, A. M., Strobel, G. A., Moore, E., Robison, R., and Sears, J. (2010).Volatile antimicrobials from Muscodor crispans, anovel endophytic fungus. Microbiology 156, 270-277. doi: 10.1099/mic.0.032540-0

Momesso, L. S., Kawano, C. Y., Ribeiro, P. H., Nomizo, A., Goldman, G. H., and Pupo, M. T. (2008). Chaetoglobosins produced by Chaetomium globosum, an endophytic fungus found in association with Viguiera robusta Gardn (Asteraceae). Quim. Nova 31, 1680-1685. doi: 10.1590/S010040422008000700015

Muñoz-Elías, E. J., and McKinney, J. D. (2005). Mycobacterium tuberculosis isocitrate lyases 1 and 2 are jointly required for in vivo growth and virulence. Nat. Med. 11, 638-644. doi: 10.1038/nm1252

Mutnick, A. H., Enne, V., and Jones, R. N. (2003). Linezolid resistance since 2001: SENTRY antimicrobial surveillance program. Ann. Pharm. 37, 769-774. doi: 10.1345/aph.1C437

Newman, D. J., and Cragg, G. M. (2012). Natural products as sources of new drugs over the 30 years from 1981 to 2010. J. Nat. Prod. 75, 311-335. doi: $10.1021 / \mathrm{np} 200906 \mathrm{~s}$

Obiang-Obounou, B. W., Kang, O. H., Choi, J. G., Keum, J. H., Kim, S. B., Mun, S. H., et al. (2011). The mechanism of action of sanguinarine against methicillin-resistant Staphylococcus aureus. J. Toxicol. Sci. 36, 277-283. doi: $10.2131 /$ jts. 36.277

Ola, A. R. B., Thomy, D., Lai, D., Broetz-Oesterhelt, H., and Proksch, P. (2013). Inducing Secondary Metabolite Production by the Endophytic Fungus Fusarium tricinctum through Coculture with Bacillus subtilis. J. Nat. Prod. 76, 2094-2099. doi: 10.1021/np400589h

Pan, J. H., Chen, Y., Huang, Y. H., Tao, Y. W., Wang, J., Li, Y., et al. (2011). Antimycobacterial activity of fusaric acid from a mangrove endophyte and its metal complexes. Arch. Pharm. Res. 34, 1177-1181. doi: 10.1007/s12272-0110716-9

Periyasamy, G., Verekar, S. A., Gohil, A. R., Mishra, P. D., Khanna, A., and Deshmukh, S. K. (2014). Antiproliferative activity of Hamigerone and Radicinol isolated from Bipolaris papendorfii. Biomed Res. Int. 2014, 7. doi: $10.1155 / 2014 / 890904$

Pinheiro, E. A. A., Carvalho, J. M., Santos, D., Diellem, C. P., Feitosa, A. de. O., Marinho, P. S. B., et al. (2013). Antibacterial activity of alkaloids produced by fungus Aspergillus sp. EJC08 isolated from medical plant Bauhinia guianensis. Nat. Prod. Res. 27, 1633-1638. doi: 10.1080/14786419.2012. 750316

Pongcharoen, W., Rukachaisirikul, V., Phongpaichit, S., and Sakayaroj, J. (2007). A new dihydrobenzofuran derivative from the endophytic fungus Botryosphaeria mamane PSU-M76. Chem. Pharm. Bull. 55, 1404-1405. doi: 10.1248/cpb.55.1404

Puri, S. C., Verma, V., Amna, T., Qazi, G. N., and Spiteller, M. (2005).An endophytic fungus from Nothapodytes foetida that produces camptothecin. J. Nat. Prod. 68, 1717-1719. doi: 10.1021/np0502802

Qin, S., Hussain, H., Schulz, B., Draeger, S., and Krohn, K. (2010). Two new metabolites, epoxydine A and B, from Phoma sp. Helv. Chim. Acta 93, 169-174. doi: 10.1002/hlca.200900199

Qin, S., Krohn, K., Floerke, U., Schulz, B., Draeger, S., Pescitelli, G., et al. (2009). Two new fusidilactones from the fungal endophyte Fusidium sp. Eur. J. Org. Chem. 2009, 3279-3284. doi: 10.1002/ejoc.200900152

Qin, S., Krohn, K., Hussain, H., Schulz, B., and Draeger, S. (2011). Pestalotheols E-H: antimicrobial metabolites from an endophytic fungus isolated from the tree Arbutus unedo. Eur. J. Org. Chem. 2011, 5163-5166. doi: 10.1002/ejoc. 201100568

Rice, L. B. (2008). Federal funding for the study of antimicrobial resistance in nosocomial pathogens: no ESKAPE. J. Infect. Dis. 197, 1079-1081. doi: $10.1086 / 533452$

Rodriguez, R., and Redman, R. (2008). More than 400 million years of evolution and some plants still can't make it on their own: plant stress tolerance via fungal symbiosis. J. Exp. Bot. 59, 1109-1114. doi: 10.1093/jxb/erm342

Rukachaisirikul, V., Arunpanichlert, J., Sukpondma, Y., Phongpaichit, S., and Sakayaroj, J. (2009). Metabolites from the endophytic fungi Botryosphaeria rhodina PSU-M35 and PSU-M114. Tetrahedron 65, 10590-10595. doi: 10.1016/j.tet.2009.10.084

Saleem, M., Tousif, M. I., Riaz, N., Ahmed, I., Schulz, B., Ashraf, M., et al. (2013). Cryptosporioptide: a bioactive polyketide produced by an endophytic fungus Cryptosporiopsis sp. Phytochemistry 93, 199-202. doi: 10.1016/j.phytochem.2013.03.018 
Schmeda-Hirschmann, G., Hormazabal, E., Rodriguez, J. A., and Theoduloz, C. (2008). Cycloaspeptide A and Pseurotin A from the Endophytic Fungus Penicillium janczewskii. Z. Naturforsch. 63C, 383-388.

Sebastianes, F. L. S., Cabedo, N., El Aouad, N., Valente, A. M. M. P., Lacava, P. T., Azevedo, J. L., et al. (2012). 3-Hydroxypropionic acid as an antibacterial agent from endophytic fungus Diaporthe phaseolorum. Curr. Microbiol. 65, 622-632. doi: 10.1007/s00284-012-0206-4

Senadeera, S. P., Wiyakrutta, S., Mahidol, C., Ruchirawat, S., and Kittakoop, P. (2012). A novel tricyclic polyketide and its biosynthetic precursor azaphilone derivatives from the endophytic fungus Dothideomycete sp. Org. Biomol. Chem. 10, 7220-7226, doi: 10.1039/c2ob25959a

Shang, Z., Li, X. M., Li, C. S., and Wang, B. G. (2012). Diverse secondary metabolites produced by marine-derived fungus Nigrospora sp. MA75 on various culture media. Chem. Biodivers. 9, 1338-1348. doi: 10.1002/cbdv. 201100216

Shao, C., Hu, G., Yang, R., Xia, X., Wang, C., She, Z., et al. (2008a). Secondary metabolites of mangrove endophytic fungus B77 in South China Sea. Zhongshan Da. Xue. Ziran Kexueban 47, 56-58.

Shao, C. L., Wang, C. Y., Deng, D. S., She, Z. G., Gu, Y. C., and Lin, Y. C. (2008b). Crystal structure of a marine natural compound, anhydrofusarubin. Chinese J. Struct. Chem. 27, 824-828.

Shiono, Y., Hatakeyama, T., Murayama, T., and Koseki, T. (2012). Polyketide metabolites from the endophytic fungus Microdiplodia sp. KS 75-1. Nat. Prod. Commun. 7, 1065-1068.

Shu, R. G., Wang, F. W., Yang, Y. M., Liu, Y. X., and Tan, R. X. (2004). Antibacterial and xanthine oxidase inhibitory cerebrosides from Fusarium sp. IFB-121, an endophytic fungus in Quercus variabilis. Lipids 39, 667-673. doi: 10.1007/s11745-004-1280-9

Siddiqui, I. N., Zahoor, A., Hussain, H., Ahmed, I., Ahmad, V. U., Padula, D., et al. (2011). Diversonol and blennolide derivatives from the endophytic fungus Microdiplodia sp.: absolute configuration of diversonol. J. Nat. Prod. 74 365-373. doi: 10.1021/np100730b

Singh, M. P., Janso, J. E., and Brady, S. F. (2007). Cytoskyrins and cytosporones produced by Cytospora sp. CR200: taxonomy, fermentation and biological activities. Mar. Drugs 5, 71-84. doi: 10.3390/md503071

Singh, M. P., Janso, J. E., Luckman, S. W., Brady, S. F., Clardy, J., Greenstein, M., et al. (2000). Biological activity of guanacastepene, a novel diterpenoid antibiotic produced by an unidentified fungus CR115. J. Antibiot. 53, 256-261. doi: 10.7164/antibiotics.53.256

Skiest, D. J. (2006). Treatment failure resulting from resistance of Staphylococcus aureus to Daptomycin. J. Clin. Microbiol. 44, 655-656. doi: 10.1128/JCM.44.2.655-656.2006

Sommart, U., Rukachaisirikul, V., Sukpondma, Y., Phongpaichit, S., Sakayaroj, J., and Kirtikara, K. (2008). Hydronaphthalenones and a dihydroramulosin from the endophytic fungus PSU-N24. Chem. Pharm. Bull. 56, 1687-1690. doi: $10.1248 /$ cpb.56.1687

Strobel, G. A., Dirksie, E., Sears, J., and Markworth, C. (2001). Volatile antimicrobials from a novel endophytic fungus. Microbiology 147, 2943-2950.

Strobel, G. A., Ford, E., Worapong, J., Harper, J. K., Arif, A. M., Grant, D. M., et al. (2002). Isopestacin, an isobenzofuranone fromPestalotiopsis microspora, possessing antifungal and antioxidant activities. Phytochemistry 60, 179-183. doi: 10.1016/S0031-9422(02)00062-6

Strobel, G. A., Knighton, B., Kluck, K., Ren, Y., Livinghouse, T., Griffin, M., et al. (2008). The production of myco-diesel hydrocarbons and their derivatives by the endophytic fungus Gliocladium roseum (NRRL 50072). Microbiology 154, 3319-3328. doi: 10.1099/mic.0.2008/022186-0

Strobel, G., and Daisy, B. (2003). Bioprospecting for microbial endophytes and their natural products. Microbiol. Mol. Biol. Rev. 67, 491-502. doi: 10.1128/MMBR.67.4.491-502.2003

Strobel, G., Yang, X., Sears, J., Kramer, R., Sidhu, R. S., and Hess, W. M. (1996). Taxol from Pestalotiopsis microspora, an endophytic fungus of Taxus wallichiana. Microbiology142, 435-440. doi: 10.1099/13500872-142-2-435

Subban, K., Subramani, R., and Johnpaul, M. (2013). A novel antibacterial and antifungal phenolic ompound from the endophytic fungus Pestalotiopsis mangiferae. Nat. Prod. Res. 27, 1445-1449. doi: 10.1080/14786419.2012. 722091

Sun, P., Huo, J., Kurtan, T., Mandi, A., Antus, S., Tang, H., et al. (2013a). Structural and stereochemical studies of hydroxyanthraquinone derivatives from the endophytic fungus Coniothyrium sp. Chirality 25, 141-148. doi: 10.1002/chir.22128
Sun, R. R., Miao, F. P., Zhang, J., Wang, G., Yin, X. L., and Ji, N. Y. (2013b). Three new xanthone derivatives from an algicolous isolate of Aspergillus wentii. Magn. Reson. Chem. 51, 65-68. doi: 10.1002/mrc.3903

Tan, R. X., and Zou, W. X. (2001). Endophytes: a rich source of functional metabolites. Nat. Prod. Rep. 18, 448-459. doi: 10.1039/b100918o

Tegos, G., Stermitz, F. R., Lomovskaya, O., and Lewis, K. (2002). Multidrug pump inhibitors uncover remarkable activity of plant antimicrobials. Antimicrob. Agents Chemother. 46, 3133-3141. doi: 10.1128/AAC.46.10.31333141.2002

Tsuchinari, M., Shimanuki, K., Hiramatsu, F., Muratama, T., Koseki, T., and Shiono, Y. (2007). Fusapyridons A and B, novel pyridone alkaloids from an endophytic fungus, Fusarium sp. YG-45. Z. Naturforsc. B Chem. Sci. 62, 1203-1207.

Verma, V. C., Lobkovsky, E., Gange, A. C., Singh, S. K., and Prakash, S. (2011). Piperine production by endophytic fungus Periconia sp. isolated from Piper longum L. J. Antibiot. 64, 427-431. doi: 10.1038/ja.2011.27

Wagenaar, M. M., and Clardy, J. (2001). Dicerandrols, new antibiotic and cytotoxic dimers produced by the fungus Phomopsis longicolla isolated from an endangered mint. J. Nat. Prod. 64, 1006-1009. doi: 10.1021/np010020u

Wang, C., Wang, J., Huang, Y., Chen, H., Li, Y., Zhong, L., et al. (2013b). Anti-mycobacterial activity of marine fungus-derived 4-deoxybostrycin and nigrosporin. Molecules 18, 1728-1740. doi: 10.3390/molecules 18021728

Wang, F. W., Ye, Y. H., Ding, H., Chen, Y. X., Tan, R. X., and Song, Y. C. (2010). Benzophenones from Guignardia sp. IFB-E028, an endophyte on Hopea hainanensis. Chem. Biodivers. 7, 216-220. doi: 10.1002/cbdv.200800353

Wang, Q. X., Bao, L., Yang, X. L., Liu, D. L., Guo, H., Dai, H. Q., et al. (2013a). Ophiobolins P-T, five new cytotoxic and antibacterial sesterterpenes from the endolichenic fungus Ulocladium sp. Fitoterapia 90, 220-227. doi: 10.1016/j.fitote.2013.08.002

Wang, Q. X., Li, S. F., Zhao, F., Dai, H. Q., Bao, L., Ding, R., et al. (2011). Chemical constituents from endophytic fungus Fusarium oxysporum. Fitoterapia 82, 777-781. doi: 10.1016/j.fitote.2011.04.002

Wang, X. J., Min, C. L., Ge, M., and Zuo, R. H. (2014). An Endophytic Sanguinarine-Producing Fungus from Macleaya cordata, Fusarium proliferatum BLH51. Curr. Microbiol. 68, 336-341. doi: 10.1007/s00284-013-0482-7

Weber, D., Sterner, O., Anke, T., Gorzalczancy, S., Martino, V., and Acevedo, C. (2004). Phomol, a new antiinflammatory metabolite from an endophyte of the medicinal plant Erythrina crista-galli. J. Antibiot. 57, 559-563. doi: 10.7164/antibiotics.57.559

Wicklow, D. T., and Poling, S. M. (2009). Antimicrobial activity of pyrrocidines from Acremoniumzeae against endophytes and pathogens of maize. Phytopathology 99, 109-115. doi: 10.1094/PHYTO-99-1-0109

Wijeratne, E. M., He, H., Franzblau, S. G., Hoffman, A. M., and Gunatilaka, A. A. (2013). Phomapyrrolidones A-C, antitubercular alkaloids from the endophytic fungus Phoma sp. NRRL 46751. J. Nat. Prod. 76, 1860-1865. doi: $10.1021 / \mathrm{np} 400391 \mathrm{p}$

Wu, S. H., Zhao, L. X., Chen, Y. W., Huang, R., Miao, C. P., and Wang, J. (2011). Sesquiterpenoids from the endophytic fungus Trichoderma sp. PR-35 of Paeonia delavayi. Chem. Biodivers. 8, 1717-1723. doi: 10.1002/cbdv.201000236

Xu, C., Wang, J., Gao, Y., Lin, H., Du, L., Yang, S., et al. (2010a). The anthracenedione compound bostrycin induces mitochondria-mediated apoptosis in the yeast Saccharomycescerevisiae. FEMS Yeast Res. 10, 297-308. doi: 10.1111/j.15671364.2010.00615.x

Xu, L., Wang, J., Zhao, J., Li, P., Shan, T., Wang, J., et al. (2010b).Beauvericin from the endophytic fungus, Fusarium redolens, isolated from Dioscorea zingiberensis and its antibacterial activity. Nat. Prod. Commun. 5, 811-814.

Ymele-Leki, P., Cao, S., Sharp, J., Lambert, K. G., McAdam, A. J., Husson, R. N., et al. (2012). A high-throughput screen identifies a new natural product with broad-spectrum antibacterial activity. PLOS ONE 7:e31307. doi: 10.1371/journal.pone. 0031307

You, X., Feng, S., Luo, S., Cong, D., Yu, Z., Yang, Z., et al. (2013). Studies on a rheinproducing endophytic fungus isolated from Rheum palmatum L. Fitoterapia 85 , 161-168. doi: 10.1016/i.fitote.2012.12.010

Zhang, B., Salituro, G., Szalkowski, D., Li, Z., Zhang, Y., Royo, I., et al. (1999). Discovery of smallmolecule insulin mimetic with antidiabetic activity in mice. Science 284, 974-981. doi: 10.1126/science.284.5416.974

Zhang, H., Ma, Y., and Liu, R. (2012). Antimicrobial additives from endophytic fungus Fusarium solani of Ficus carica. Appl. Mech. Mater. 178181(Pt 2, Sustainable Environment and Transportation), 783-786. doi: 10.4028/www.scientific.net/AMM.178-181.783 
Zhang, H. W., Song, Y. C., and Tan, R. X. (2006). Biology and chemistry of endophytes. Nat. Prod. Rep. 23, 753-771. doi: 10.1039/b609472b

Zhang, W., Draeger, S., Schulz, B., and Krohn, K. (2009). Ring B aromatic steroids from an endophytic fungus, Colletotrichum sp. Nat. Prod. Commun. 4, 1449-1454.

Zhang, W., Krohn, K., Draeger, S., and Schulz, B. (2008a). Bioactive isocoumarins isolated from the endophytic fungus Microdochium bolleyi. J. Nat. Prod. 71, 1078-1081 doi: 10.1021/np800095g

Zhang, W., Krohn, K., Zia, U., Florke, U., Pescitelli, G., Di, B., L., et al. (2008b). New mono- and dimeric members of the secalonic acid family: blennolides A-G isolated from the fungus Blennoria sp. Chemistry 14, 4913-4923. doi: 10.1002/chem. 200800035

Zhao, J., Mou, Y., Shan, T., Li, Y., Zhou, L., Wang, M., et al. (2010). Antimicrobial metabolites from the endophytic fungus Pichia guilliermondii isolated from Paris polyphylla var. yunnanensis. Molecules 15, 7961-7970. doi: 10.3390/molecules 15117961

Zhu, F., Chen, G., Chen, X., Huang, M., and Wan, X. (2011). Aspergicin, a new antibacterial alkaloid produced by mixed fermentation of two marine-derived mangrove epiphytic fungi. Chem. Nat. Comp. 47, 767-769. doi: 10.1007/s10600011-0053-8
Zou, W. X., Meng, J. C., Lu, H., Chen, G. X., Shi, G. X., Zhang, T. Y., et al. (2000). Metabolites of Colletotrichum gloeosporioides, an endophytic fungus in Artemisia mongolica. J. Nat. Prod. 63, 1529-1530. doi: 10.1021/np000204t

Conflict of Interest Statement: The authors declare that the research was conducted in the absence of any commercial or financial relationships that could be construed as a potential conflict of interest.

Received: 30 September 2014; accepted: 01 December 2014; published online: 08 January 2015.

Citation: Deshmukh SK, Verekar SA and Bhave SV (2015) Endophytic fungi: a reservoir of antibacterials. Front. Microbiol. 5:715. doi: 10.3389/fmicb.2014.00715

This article was submitted to Microbial Physiology and Metabolism, a section of the journal Frontiers in Microbiology.

Copyright $\odot 2015$ Deshmukh, Verekar and Bhave. This is an open-access article distributed under the terms of the Creative Commons Attribution License (CC BY). The use, distribution or reproduction in other forums is permitted, provided the original author(s) or licensor are credited and that the original publication in this journal is cited, in accordance with accepted academic practice. No use, distribution or reproduction is permitted which does not comply with these terms. 